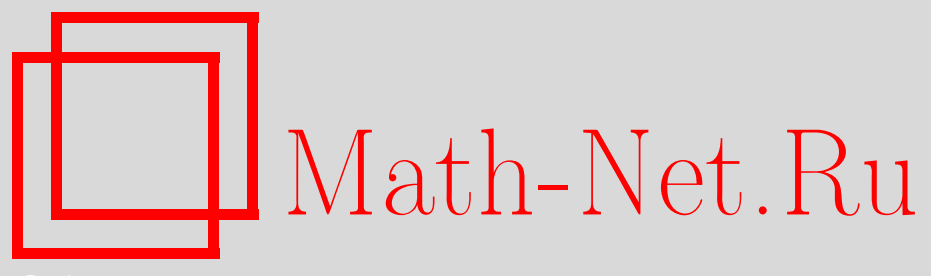

А. В. Болсинов, А. В. Борисов, И. С. Мамаев, Топология и устойчивость интегрируемых систем, УМН, 2010, том 65, выпуск 2, 71-132

DOI: https://doi.org/10.4213/rm9346

Использование Общероссийского математического портала Math-Net.Ru подразумевает, что вы прочитали и согласны с пользовательским соглашением http://www . mathnet.ru/rus/agreement

Параметры загрузки:

IP: 3.85 .7 .115

26 апреля 2023 г., 11:07:44

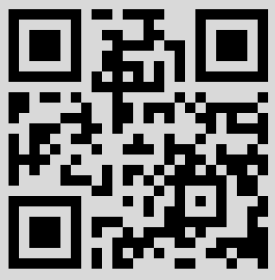




\title{
Топология и устойчивость интегрируемых систем
}

\author{
А. В. Болсинов, А. В. Борисов, И. С. Мамаев
}

В работе предложен общий топологический подход к исследованию устойчивости периодических решений интегрируемых динамических систем с двумя степенями свободы. Развиваемые методы проиллюстрированы на примерах нескольких интегрируемых задач, связанных с классическими уравнениями Эйлера-Пуассона, движением твердого тела в жидкости, а также динамикой газообразных расширяющихся эллипсоидов. Данные топологические методы позволяют также отыскивать невырожденные периодические решения интегрируемых систем, что является особенно актуальным в тех случаях, когда общее решение, например, при помощи разделения переменных, неизвестно.

Библиография: 82 названия.

Ключевые слова: топология, устойчивость, периодическая траектория, критическое множество, бифуркационное множество, бифуркационная диаграмма.

\section{СоДЕРЖАНИЕ}

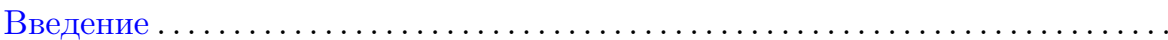

1. Постановка задачи, предположения, примеры .................... 77

1.1. Интегрируемые системы, теорема Лиувилля, основные предпо-

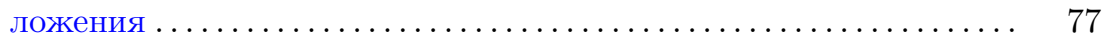

1.2. Гамильтоновы системы на пуассоновых многообразиях ....... 79

1.3. Конформно-гамильтоновы системы ..................... 79

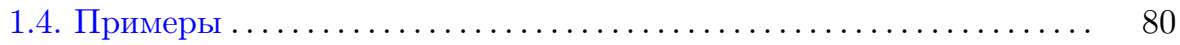

2. Бифуркационная диаграмма и типы периодических траекторий ...... 81

2.1. Бифуркационная диаграмма и типы интегральных многообразий 81

2.2. Критические и некритические периодические траектории...... 83

2.3. Невырожденные периодические траектории ............. 86

2.4. Бифуркационная диаграмма случая Горячева-Чаплыгина ..... 92

2.5. Бифуркационная диаграмма случая Клебша ............... 96

Работа выполнена при поддержке Федеральной целевой программы "Научные и научнопедагогические кадры инновационной России" (код проекта 2009-1.5-503-004-019). Работа А.В. Болсинова выполнена при поддержке программы "Ведущие научные школы" (грант НШ-660.2008.1). Работа И. С. Мамаева выполнена при поддержке гранта Президента Российской Федерации для поддержки молодых докторов наук (код проекта МД-5239.2008.1).

(С) А. В. Болсинов, А. В. Борисов, И. С. МАмаев, 2010 
3. Устойчивость периодических траекторий ....................... 101

3.1. Случай некритических периодических траекторий ........... 101

3.2. Случай невырожденных периодических траекторий........... 103

3.3. Общий критерий устойчивости. І . . . . . . . . . . . . . . . . 105

3.4. Устойчивость семейств периодических траекторий и бифуркационная диаграмма.............................. 106

3.5. Общий критерий устойчивости. II. Бифуркационный комплекс . 108

4. Бифуркационные комплексы систем динамики твердого тела ....... 113

4.1. Бифуркационный комплекс волчка Горячева-Чаплыгина ... . . . 113

4.2. Бифуркационные комплексы случая Клебша................ 113

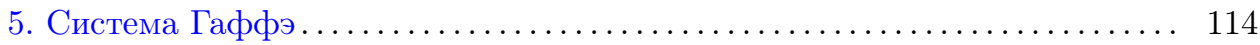

5.1. Случай $c=0$ (интеграл третьей степени) ............... 117

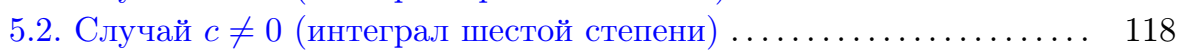

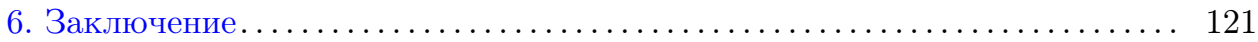

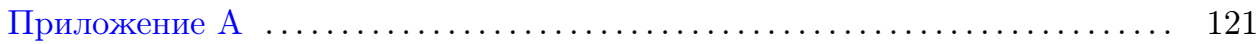

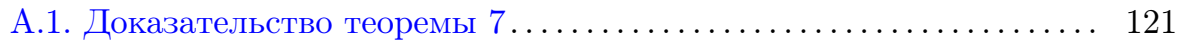

А.2. Методы проверки невырожденности .................... 124

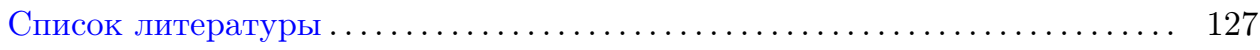

\section{Введение}

В настоящей работе развивается общий подход к проблеме устойчивости для интегрируемых динамических систем, основанный на систематическом применении методов топологического анализа. Во многом этот подход является естественным развитием идей В. В. Козлова о применении топологических методов к качественному анализу динамики механических систем. Эти методы особенно наглядно продемонстрировали свою продуктивность в задачах динамики твердого тела. Такой подход, в частности, позволяет наиболее эффективным образом (в сравнении со стандартными методами) определять устойчивость в случае интегрируемых гамильтоновых систем с двумя степенями свободы важнейшего класса динамических систем, представленного многочисленными примерами из механики и других прикладных областей. Нашей главной целью, в этой связи, было сделать доступным широкому кругу исследователей математически строгое изложение общей теории и результатов. Мы надеемся, что развиваемые здесь методы окажутся полезными и более удобными при исследованиях на практике для специалистов, изучающих конкретные механические системы, где вопросы устойчивости имеют принципиальное значение.

Прежде чем обсуждать основные аспекты этой работы, сделаем ряд исторических комментариев относительно происхождения используемых методов.

Как известно, первым, кто глубоко осознал концептуальное значение качественных, топологических методов в динамике в целом, был А. Пуанкаре. Его общий подход в дальнейшем получил развитие в огромном количестве работ по качественной теории дифференциальных уравнений, общей теории динамических систем, эргодическим проблемам и др. Применительно к анализу механических систем топологические методы, восходящие к Пуанкаре, были развиты С. Смейлом в работе “Топология и механика" (1970) [1]. Изложенные 
Смейлом идеи не только нашли свое применение во многих работах по топологическому анализу конкретных механических систем, но и оказали мощное стимулирующее воздействие на развитие дифференциальной топологии как отдельной дисциплины.

Следует, однако, отметить, что в таком аспекте, как анализ устойчивости динамических систем, топологические методы имели до настоящего времени довольно ограниченное применение. Мы здесь будем рассматривать устойчивость периодических решений интегрируемых систем, которые, как известно, являются одним из центральных объектов исследования в механике. Предложенный в этой работе общий метод позволяет систематически находить такие решения и полностью, в строгом виде, решать задачу об их устойчивости.

Обсуждаемые здесь вопросы исторически берут свое начало из работы семинаров, проводившихся в 1980-х годах на механико-математическом факультете Московского университета под руководством В. В. Козлова и А. Т. Фоменко. Активная деятельность этих семинаров в тот период во многом была инициирована фундаментальными работами В. В. Козлова о топологических препятствиях к интегрируемости натуральных механических систем [2]-[4]. В этих работах было показано, что сложное топологическое строение конфигурационного пространства несовместимо с интегрируемостью уравнений движения соответствующей механической системы. Отсюда естественно возникает следующая задача, представляющая для механиков большой интерес: каким образом фазовое пространство интегрируемой динамической системы расслаивается на инвариантные торы (торы Лиувилля)? Для решения в той или иной степени этой проблемы со времен Пуанкаре использовались бифуркационные диаграммы, но они, как известно, имеют неинвариантный характер и зависят от выбора конкретной комбинации первых интегралов. В.В.Козлов предложил, опираясь на бифуркационные диаграммы, строить более инвариантные объекты. Стоит также отметить, что с обсуждением этого круга проблем связан один из наиболее плодотворных периодов механико-математического факультета, объединивший топологов и механиков в активном диалоге.

Эти идеи оказали серьезное влияние на формирование теории топологических инвариантов интегрируемых систем, поначалу ставившей одной из основных своих целей изучение топологических препятствий к интегрируемости гамильтоновых систем. Однако уже в первых работах в этом направлении [5]-[7] намечена разработка общего, фундаментального подхода к топологическому описанию интегрируемых систем. Изложение этого подхода содержится в известной книге А. Т. Фоменко [5].

Несколько ранее независимое развитие вопросов топологического анализа интегрируемых систем было начато М.П. Харламовым и Т.И. Погосяном [8]-[12]. Эти работы изначально отличались более практической направленностью, ставя во главу угла детальный качественный анализ важнейших систем динамики твердого тела и развитие топологических методов такого анализа. В связи с изучением поведения траекторий таких систем, определением областей возможного движения, исследовалась фазовая топология их решений (рассматривались бифуркационные диаграммы интегрального отображения, топологический тип интегральных многообразий, перестройки торов Лиувил- 
ля). На этом пути М. П. Харламовым был собран интересный и разнообразный фактический материал о конкретных топологических феноменах в конкретных классических задачах. Методы и результаты этих исследований изложены в монографии [13].

Интересно отметить, что книги [13] и [5] вышли в свет одновременно (1988), при этом во введении к [13] автор отмечает, что «наличие явных закономерностей в многочисленных, иногда весьма нетривиальных, примерах бифуркаций позволяет надеяться на возникновение в ближайшем будущем общей “теории Морса интегрируемых систем"». Как мы видим в [5], к этому моменту общая теория фактически уже была создана. Опираясь на новую теорию [5]-[7] и имеющийся вычислительный опыт [8]-[13], впоследствии был выполнен подробный топологический анализ большого числа классических интегрируемых систем механики (наиболее полная информация содержится в [14]). Однако следует отметить, что подобное рассмотрение частных случаев многими было воспринято лишь как иллюстрация общей теории.

Как мы видим, процесс развития методов топологического анализа динамических систем происходил в двух независимых, идейно различных направлениях - от подробного исследования конкретных примеров к общей теории, с одной стороны, и от создания общей теории к рассмотрению примеров, с другой стороны.

В рамках теории топологических инвариантов была осуществлена полная топологическая классификация систем с двумя степенями свободы на изоэнергетических поверхностях [14] и вычислены топологические классифицирующие инварианты для большого числа классических интегрируемых систем с двумя степенями свободы [14]-[22].

Подчеркнем, что, помимо этих важных практических результатов, наиболее существенным является то, что эта теория выработала общий качественный взгляд на строение произвольной интегрируемой системы. Это исключительно важно, когда мы работаем с конкретными примерами: пользуясь общими результатами, мы обычно можем сделать необходимые нам выводы по сравнительно небольшому объему информации (например, устойчивость определяется фактически по расположению исследуемых решений на бифуркационной диаграмме). Если же не пользоваться никакими общими теоремами, то топологический анализ системы связан с довольно громоздкими вычислениями.

Тем не менее, следует отметить, что данная теория пока не нашла систематического, эффективного применения в среде механиков и других специалистов-прикладников. Практический смысл вычисления топологических классифицирующих инвариантов для механических систем пока остается неясным. С одной стороны, имеется инструмент, позволяющий детально описывать топологическое строение интегрируемой системы, однако часто отсутствует полное понимание того, с какими наблюдаемыми динамическими эффектами связаны различные детали этого строения.

Очевидно, что в деле сближения общей теории с приложениями совершенно необходимы усилия по ее осмыслению и со стороны специалистов-практиков. Такие попытки, разумеется, предпринимаются (см., например, [23]-[25]). На этом пути едва ли можно обойтись без того, чтобы совместными усилиями спе- 
циалистов по общей теории и исследователей реальных механических систем изложить эту теорию в более доступном виде, ориентированном на широкий круг специалистов. Попытку подобного рода представляет собой настоящая работа, в которой идеи теории обсуждаются с точки зрения проблемы устойчивости.

Возможно, вычисление топологических инвариантов имеет более важное значение в прикладных задачах квантового характера. Бифуркационные диаграммы таких задач, возникающих в основном из физической химии, используются в работах Б. Жилинского (см., например, [26]).

Отметим, что историческое описание методов топологического анализа интегрируемых систем, приведенное выше, сильно упрощено. Приведем здесь список ряда авторов, внесших, по нашему мнению, наибольший вклад в развитие этого направления: Дюистермаат [27], Элиассон [28], Лерман, Уманский [29], Кушман, Бейтс [30], Дельзант [31]. Особо отметим фундаментальные результаты по особенностям интегрируемых (в том числе многомерных) систем Нгуен Тьен Зунга [32], [33], которые выходят далеко за рамки тех идей теории топологических инвариантов интегрируемых систем, которые обсуждаются ниже.

Заметим также, что, помимо описанного выше, своей фундаментальной работой [2] В.В. Козлов заложил еще одно направление, связанное с топологическими препятствиями к интегрируемости. Его теоремы были обобщены И. А. Таймановым на многомерный случай [34], [35] и в дальнейшем были развиты в значительных работах С. В. Болотина [36], [37], А. В. Болсинова, И. А. Тайманова [38], Г. Паттернайна [39], Л. Батлера [40], [41] и др.

В данной работе ставится задача поиска устойчивых периодических решений интегрируемых гамильтоновых систем с двумя степенями свободы. Очень часто при анализе устойчивости периодических решений и неподвижных точек не делают различия между интегрируемыми и неинтегрируемыми системами и пользуются общими методами, основанными на вычислении мультипликаторов, нормализующих преобразованиях Биркгофа, изучении областей резонансов и так называемых связок интегралов (см., например, [42]-[48]).

Как будет показано ниже, предлагаемый нами метод, естественным образом используя интегрируемость системы, позволяет быстрым и наглядным образом определять устойчивость в тех случаях, когда использование общих стандартных методов является довольно затруднительным.

Специально отметим, что методы, от которых мы отталкиваемся в нашем исследовании, фактически уже были введены в предшествующих работах по топологическому анализу интегрируемых гамильтоновых систем. Таким образом, результаты настоящей работы должны быть совершенно ясны для специалистов в этой области; возможно, они покажутся им даже самоочевидными. Тем не менее, до настоящего времени проблемы исследования устойчивости в интегрируемых системах с топологической точки зрения не выдвигались в литературе на передний план. Здесь мы выполнили их систематический анализ, в ходе которого оказалось, что для строгого обоснования устойчивости топологическими методами необходимо доказать ряд дополнительных весьма естественных утверждений и теорем общего характера (так называемые то- 
пологические критерии устойчивости). Тем самым в работе устранен ряд имевшихся пробелов.

Обобщая понятие бифуркационной диаграммы, мы вводим новый объект так называемый бифуркационный комплекс, который является простым, наглядным и естественным топологическим инвариантом интегрируемой системы. Его главное преимущество связано с упрощениями, которые достигаются при анализе и представлении результатов о существовании и устойчивости периодических решений интегрируемых систем. Построение этого инварианта дает возможность не только ответить на вопрос об устойчивости каких-то конкретных траекторий, но сразу описать все устойчивые траектории.

Для иллюстрации нашего метода мы построили бифуркационные комплексы для двух классических задач динамики твердого тела - волчка Горячева-Чаплыгина и системы Клебша (интегрируемого случая движения твердого тела в жидкости), а также для системы Гаффэ (описывающей динамику газового эллипсоида, заполненного одноатомным идеальным газом). В первых двух задачах критические периодические решения хорошо известны, и мы лишь систематизировали результаты по их устойчивости. На примере системы Гаффэ наш метод был апробирован в полной мере. Дополнительный интеграл в этой задаче может быть как третьей, так и шестой степени; аналитическим путем он был найден Б. Гаффэ. Эти интегралы настолько сложны, что качественную информацию о решении этой системы получить крайне затруднительно. С помощью нашего метода мы нашли новые периодические решения этой задачи, которые описывают пульсирующие расширяющиеся газовые эллипсоиды и, возможно, представляют интерес в астрофизике. Кроме того, была полностью исследована устойчивость этих решений.

Стоит отметить, что для таких сложных случаев, как система Гаффэ, где разделение переменных представляет собой трудновыполнимую задачу либо неизвестно вообще, применение нашего метода пока является безальтернативным.

В недавно опубликованной работе [49] этот алгоритм был применен для нахождения и определения устойчивости частных решений задачи из неголономной механики.

Окончательно сформулируем кратко основные цели, которые мы преследовали при написании этой работы.

- Облегчить поиск и классификацию периодических решений в интегрируемых системах.

- Облегчить анализ устойчивости периодических решений в этих системах, в частности, исключить вычисление монодромии и т. п.

- Обосновать результаты об устойчивости периодических решений, полученные при помощи бифуркационной диаграммы.

- Дать наглядное представление результатов об устойчивости при помощи использования бифуркационного комплекса интегрируемой системы.

- Дать полное описание динамики системы в окрестности особых (критических) периодических траекторий. 
- Расширить область применения топологических методов для анализа устойчивости конформно-гамильтоновых интегрируемых систем, в частности, интегрируемых неголономных систем.

\section{1. Постановка задачи, предположения, примеры}

\section{1. Интегрируемые системы, теорема Лиувилля, основные пред-} положения. Пусть задана гамильтонова система с двумя степенями свободы, которая в канонических переменных $\mathbf{q}=\left(q_{1}, q_{2}\right), \mathbf{p}=\left(p_{1}, p_{2}\right)$ задается в виде

$$
\dot{\mathbf{q}}=\frac{\partial H}{\partial \mathbf{p}}, \quad \dot{\mathbf{p}}=-\frac{\partial H}{\partial \mathbf{q}},
$$

где $H(\mathbf{q}, \mathbf{p})$ - гамильтониан. Будем предполагать, что система обладает дополнительным первым интегралом $F(\mathbf{q}, \mathbf{p})$ и, следовательно, является интегрируемой. Здесь и далее точку на многообразии и фазовые переменные мы будем обозначать через $\mathbf{x}$, в частности, в данном случае $\mathbf{x}=(\mathbf{p}, \mathbf{q})$.

Напомним теорему Лиувилля-Арнольда, которая описывает поведение таких систем в ситуации общего положения (см., например, [14], [50], [51]).

Теорема 1. Предположим, что на симплектическом многообразии $\mathscr{M}^{4}$ задана пара функций $H(\mathbf{x}), F(\mathbf{x})$ в инволющии, т.е.

$$
\{H, F\}=0,
$$

тогда уравнения (1) интегрируются в квадратурах.

Пусть $\mathscr{M}_{h, f}=\left\{\mathbf{x} \in \mathscr{M}^{\mathbf{4}} \mid H=h, F=f\right\}-$ совместный уровень первых интегралов. Если на $\mathscr{M}_{h, f}$ функиии $H$ и $F$ независимы, то

1) $\mathscr{M}_{h, f}$ - гладкое многообразие, инвариантное относителъно фазового потока системь (1);

2) всякая компактная связная компонента поверхности $\mathscr{M}_{h, f}$ диффеоморфна двумерному тору;

3) в окрестности связной компактной компоненты $\mathscr{M}_{h, f}$ можно выбрать переменные действие-угол $\mathbf{I}=\left(I_{1}, I_{2}\right), \boldsymbol{\varphi}=\left(\varphi_{1}, \varphi_{2}(\bmod 2 \pi)\right)$, для которьх $H(\mathbf{q}, \mathbf{p})=H(\mathbf{I})$, и система (1) представляется в виде

$$
\dot{\mathbf{I}}=\frac{\partial H}{\partial \boldsymbol{\varphi}}=0, \quad \dot{\boldsymbol{\varphi}}=\frac{\partial H}{\partial \mathbf{I}}=\omega(\mathbf{I}) .
$$

Величины $\omega_{1}(\mathbf{I}), \omega_{2}(\mathbf{I})$ могут быть вычислены как функции исходных интегралов движения, т. е. $\omega_{i}(H, F), i=1,2$, и называются частотами движения.

Таким образом, в компактном случае все фазовое пространство $\mathscr{M}^{4}$ расслоено на инвариантные торы и особые слои, объединяющиеся в различные семейства. Можно выделить четыре существенно различных класса траекторий: периодические, квазипериодические, неподвижные точки и асимптотические (т.е. траектории, приближающиеся к периодическим решениям и неподвижным точкам системы (1)).

Наша цель: выделить периодические решения (замкнутые траектории) системы (1) и исследовать их (орбитальную) устойчивость. 


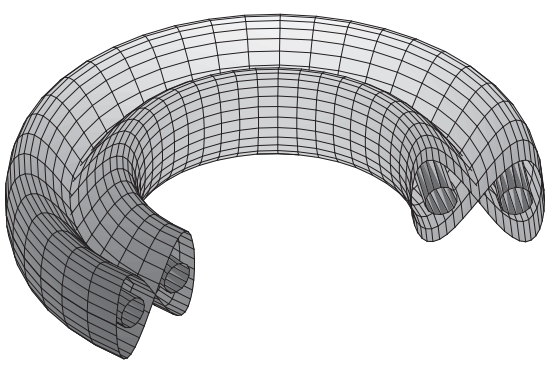

Рис. 1. Лиувиллевы торы и особое многообразие, примыкающее к неустойчивому периодическому решению

Всюду ниже мы будем предполагать выполненными следующие естественные условия.

$1^{\circ}$. Все рассматриваемые обгекты являются вещественно аналитическими. (Это предположение в конкретных задачах почти всегда выполнено. Оно значительно облегчает многие доказательства и во многих ситуациях является существенным.)

$2^{\circ}$. Совместные поверхности уровня гамильтониана и интеграла комnактны. (В частности, как отмечалось выше, регулярные интегральные поверхности являются двумерными торами.)

$3^{\circ}$. Рассматриваемая система нерезонансна в том смысле, что на почти всех инвариантных торах траектория системы задает всюду плотную обмотку.

Напомним также (см. [52]), что решение $\mathbf{x}_{0}(t), t_{0} \leqslant t<+\infty$, системы (1) называется орбитально устойчивым при $t \rightarrow+\infty$, если положительные (полу)траектории всех решений $\mathbf{x}(t), t_{0} \leqslant t<+\infty$, достаточно близкие в начальный момент к решению $\mathbf{x}_{0}(t)$, в дальнейшем содержатся в $\varepsilon$-окрестности положительной полутраектории исходного решения $\mathbf{x}_{0}(t)$, где $\varepsilon>0$ произвольно мало (см. рис. 2).

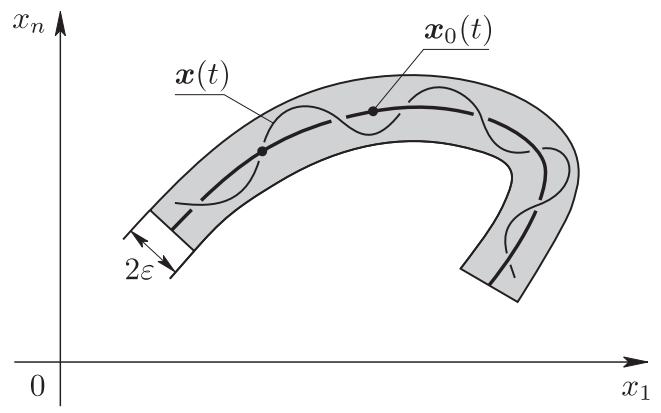

Рис. 2

В силу свойства непрерывной зависимости решений от начальных данных (см. [52]), наличие орбитальной устойчивости решения $\mathbf{x}_{0}(t), a<t<\infty$, не 
зависит от выбора начального момента $t_{0} \in(a, \infty)$, поэтому можно говорить об орбитальной устойчивости всей траектории.

Прежде всего отметим, что, в отличие от случая общих гамильтоновых систем, в интегрируемой ситуации мы имеем гораздо больше информации о системе благодаря наличию дополнительной структуры - интеграла и определяемого им слоения фазового пространства на инвариантные интегральные подмногообразия. Неудивительно поэтому, что вопрос об устойчивости в случае интегрируемой системы может быть решен технически гораздо более простыми средствами, чем те, которые обычно применяются к общим задачам.

1.2. Гамильтоновы системы на пуассоновых многообразиях. В приложениях, как правило, встречаются более сложные системы, для которых фазовое пространство является пуассоновым многообразием $\mathscr{M}$ со скобкой Пуассона

$$
\left\{x_{i}, x_{j}\right\}=J_{i j}(\mathbf{x}),
$$

где $\mathbf{x}=\left(x_{1}, \ldots, x_{n}\right)$ - некоторые координаты на $\mathscr{M}$ [14], [51]. Как и выше, будем предполагать, что пуассонова структура $\mathbf{J}=\left(J_{i j}(\mathbf{x})\right)$ является аналитической.

Гамильтонова система на $\mathscr{M}$

$$
\dot{x}_{i}=\left\{x_{i}, H\right\}, \quad i=1, \ldots, n,
$$

где $H(\mathbf{x})$ - функция Гамильтона, является естественным обобщением системы (1), рассмотренной выше.

Как известно [14], [51], пуассоново многообразие расслаивается на симплектические листы, которые являются симплектическими многообразиями. В данном случае мы будем предполагать, что симплектический лист четырехмерен $($ т. е. $\operatorname{rank} \mathbf{J}=4)$ и функции Казимира $K_{1}, \ldots, K_{m}$ - аналитические. Симплектический лист обозначим как

$$
\mathscr{M}_{c}=\left\{\mathbf{x} \mid K_{1}(\mathbf{x})=c_{1}, \ldots, K_{m}(\mathbf{x})=c_{m}\right\} .
$$

При ограничении системы (3) на симплектический лист $\mathscr{M}_{c}$ получим обычную двухстепенную гамильтонову систему, аналогичную (1), с гамильтонианом $H_{c}=\left.H\right|_{\mathscr{M}_{c}}$, который явно зависит от величин $c_{1}, \ldots, c_{m}$. Таким образом, в данном случае мы получаем семейство гамильтоновых систем, зависящих от параметров $\mathbf{c}=\left(c_{1}, \ldots, c_{m}\right)$ - констант функций Казимира.

Мы будем предполагать, что система (3) допускает дополнительный первый интеграл $F(\mathbf{x})$, по крайней мере при некоторых значениях параметра с. Если система имеет дополнительный интеграл на всем $\mathscr{M}$, то она интегрируема на всех симплектических листах, в то же время возможны ситуации, когда система интегрируема лишь на отдельных листах.

1.3. Конформно-гамильтоновы системы. Укажем еще один класс систем, для которых могут быть применены методы анализа устойчивости, развиваемые в этой работе. Конформно-гамильтоновы системы, которые также определены на пуассоновом многообразии, могут быть представлены в виде

$$
\dot{x}_{i}=N(\mathbf{x})\left\{x_{i}, H\right\}
$$


где $\{\cdot, \cdot\}$ - пуассонова структура, а приводящий множитель $N(\mathbf{x})$ является строго положительной функцией на $\mathscr{M}$. Как и выше, ограничивая систему на симплектические листы, получим семейство конформно-гамильтоновых систем, зависящих от параметров с.

Заменой времени

$$
N(\mathbf{x}) d t=d \tau \quad(N>0)
$$

конформно-гамильтоновы системы приводятся к гамильтоновой форме, причем все результаты об орбитальной устойчивости остаются справедливыми для таких систем.

ЗАмЕЧАНИЕ. В примерах в форме конформно-гамильтоновых систем представляются многие интегрируемые системы неголономной механики [53], [54].

1.4. Примеры. Рассмотрим теперь два примера интегрируемых систем случай Клебша и случай Горячева-Чаплыгина, с помощью которых мы будем иллюстрировать методы и конструкции, применяемые в работе. Обе системы это классические интегрируемые задачи динамики твердого тела, которые позволяют продемонстрировать большинство возможных ситуаций при анализе устойчивости периодических решений интегрируемых систем.

Фазовое пространство рассматриваемых систем является шестимерным пространством с координатами $\mathbf{M}=\left(M_{1}, M_{2}, M_{3}\right)$ и $\gamma=\left(\gamma_{1}, \gamma_{2}, \gamma_{3}\right)$ и со скобкой Ли-Пуассона, определяемой алгеброй $e(3)$ :

$$
\left\{M_{i}, M_{j}\right\}=\varepsilon_{i j k} M_{k}, \quad\left\{M_{i}, \gamma_{j}\right\}=\varepsilon_{i j k} \gamma_{k}, \quad\left\{\gamma_{i}, \gamma_{j}\right\}=0 .
$$

Уравнения движения (3) представляются в виде

$$
\dot{\mathbf{M}}=\mathbf{M} \times \frac{\partial H}{\partial \mathbf{M}}+\gamma \times \frac{\partial H}{\partial \gamma}, \quad \dot{\gamma}=\gamma \times \frac{\partial H}{\partial \mathbf{M}} .
$$

Скобка Пуассона (4) имеет две квадратичные функции Казимира

$$
K_{1}=(\gamma, \gamma), \quad K_{2}=(\mathbf{M}, \gamma)
$$

Для рассматриваемых систем без ограничения общности можно полагать $K_{1}=1$, так что интересующие нас симплектические листы параметризуются одним параметром $\mathscr{M}_{c}=\{\mathbf{M}, \boldsymbol{\gamma} \mid(\boldsymbol{\gamma}, \boldsymbol{\gamma})=1,(\mathbf{M}, \boldsymbol{\gamma})=c\}$.

ЗАмечАниЕ. Симплектические листы $\mathscr{M}_{c}$ диффеоморфны $T S^{2}$.

Случай Горячева-Чаплыгина определяется гамильтонианом и интегралом вида

$$
H=\frac{1}{2}\left(M_{1}^{2}+M_{2}^{2}+4 M_{3}^{2}\right)-\mu \gamma_{1}, \quad F=M_{3}\left(M_{1}^{2}+M_{2}^{2}\right)+\mu M_{1} \gamma_{3} .
$$

В этом случае $H$ и $F$ коммутируют лишь при выполнении равенства $(\mathbf{M}, \boldsymbol{\gamma})=0$ (т. е. $\left.\left.\{H, F\}\right|_{(\mathbf{M}, \gamma)=0}=0\right)$, поэтому система Горячева-Чаплыгина интегрируема лишь на листе $\mathscr{M}_{0}$. 
Случай Клебша определяется гамильтонианом и интегралом следующего вида:

$$
H=\frac{1}{2}(\mathbf{M}, \mathbf{A} \mathbf{M})+\frac{1}{2} \mu\left(\boldsymbol{\gamma}, \mathbf{A}^{-1} \gamma\right), \quad F=(\mathbf{M}, \mathbf{M})-\frac{\mu}{\operatorname{det} \mathbf{A}}(\gamma, \mathbf{A} \gamma),
$$

где $\mathbf{A}=\operatorname{diag}\left(a_{1}, a_{2}, a_{3}\right)$.

Как несложно проверить, эти функции коммутируют во всем фазовом пространстве (т. е. $\{H, F\} \equiv 0)$, поэтому интегрируемая система Клебша определена на всем семействе симплектических листов $\mathscr{M}_{c}$.

\section{2. Бифуркационная диаграмма и типы периодических траекторий}

\section{1. Бифуркационная диаграмма и типы интегральных многообра-} зий. Через $\Phi=(H, F): \mathscr{M}^{4} \rightarrow \mathbb{R}^{2}$ мы обозначим интегральное отображение интегрируемой системы:

$$
\mathbf{x} \mapsto \Phi(\mathbf{x})=(H(\mathbf{x}), F(\mathbf{x})) \in \mathbb{R}^{2} .
$$

В некоторых работах это отображение называется также отображением энергии-момента, либо просто отображением момента.

Определим ряд объектов, играющих важную роль в исследовании периодических решений:

1) область возможных движений (ОВД) $\Phi(\mathscr{M})$ - полный образ фазового пространства на плоскости первых интегралов $(h, f)$ (всякой точке $(h, f) \in$ $\Phi(\mathscr{M})$ соответствует интегральное многообразие системы $\mathscr{M}_{h, f}=\{H(\mathbf{x})=h$, $F(\mathbf{x})=f\}$, которое, вообще говоря, может содержать несколько связных компонент);

2) множество критических точек $S$ интегрального отображения

$$
S=\{\mathbf{x} \in \mathscr{M} \mid \operatorname{rank} \mathrm{d} \Phi(\mathbf{x})<2\}
$$

3) бифуркационное множество первых интегралов $\Sigma$ - образ множества критических точек $\Sigma=\Phi(S)$, т. е. значения первых интегралов, соответствующие критическим точкам интегрального отображения.

ЗАмЕчАНИЕ. Во многих работах под областью возможных движений понимается проекция интегрального многообразия $\mathscr{M}_{h, f}$ на конфигурационное пространство. Мы употребляем этот термин в другом смысле.

Очевидно, что любая траектория системы, пересекающаяся с $S$, целиком лежит в $S$. Следовательно, $S$ - инвариантное множество, целиком заполненное критическими траекториями.

Множество критических точек является объединением двух непересекающихся (инвариантных) подмножеств, различающихся значением rank $\mathrm{d} \Phi$ :

$$
\begin{gathered}
S=S_{0} \cup S_{1}, \\
S_{0}=\{\mathbf{x} \in \mathscr{M} \mid \operatorname{rankd} \Phi(\mathbf{x})=0\}, \quad S_{1}=\{\mathbf{x} \in \mathscr{M} \mid \operatorname{rank} d \Phi(\mathbf{x})=1\} .
\end{gathered}
$$


В типичной ситуации $S_{0}$ - это отдельные точки, а $S_{1}$ - однопараметрическое семейство (или несколько семейств) замкнутых кривых, которые либо являются периодическими решениями исходной системы (1), либо целиком состоят из неподвижных точек системы (1), либо включают в себя асимптотические траектории, входящие в неподвижные точки.

Образы соответствующих множеств на плоскости первых интегралов будем обозначать аналогичным образом:

$$
\Sigma_{0}=\Phi\left(S_{0}\right), \quad \Sigma_{1}=\Phi\left(S_{1}\right)
$$

Отметим, что, хотя множества $S_{0}$ и $S_{1}$ не пересекаются в $\mathscr{M}^{4}$, их образы $\Sigma_{0}$ и $\Sigma_{1}$, как правило, будут иметь непустое пересечение. Так происходит, например, в том случае, когда $S_{1}$ содержит асимптотическую траекторию, входящую в некоторую неподвижную точку из множества $S_{0}$. Ясно, что при интегральном отображении $\Phi$ образы этой траектории и неподвижной точки будут совпадать.

ОПРЕДЕЛЕНИЕ. Бифуркационной диаграммой интегрируемой системы будем называть изображенную на плоскости первых интегралов $(h, f)$ область возможных движений $\Phi(\mathscr{M})$ совместно с образом критического множества $\Sigma$ и указанием образов $\Sigma_{0}$ и $\Sigma_{1}$.

ЗАмЕчАНИЕ. Во многих работах бифуркационной диаграммой называют только бифуркационное множество $\Sigma$ (зачастую даже без выделения подмножества $\Sigma_{0}$ ). Здесь мы пользуемся несколько иной терминологией.

В типичной ситуации $\Sigma_{0}$ представляет собой набор точек, а $\Sigma_{1}$ - набор гладких кривых на плоскости (см. рис. 6-9).

Как правило, прообраз бифуркационного множества $\Phi^{-1}(\Sigma)$ не совпадает с критическим множеством $S$, так как содержит дополнительно особые многообразия, примыкающие к неустойчивым периодическим решениям и неустойчивым неподвижным точкам.

Точки $(h, f)$ на бифуркационной диаграмме определяют различные типы интегральных многообразий $\mathscr{M}_{h, f}$ и траекторий системы. Отметим, однако, что если мы хотим использовать бифуркационную диаграмму для того, чтобы сделать выводы о самой системе, то необходимо соблюдать определенную осторожность: бифуркационная диаграмма часто помогает угадать правильный ответ, но без дополнительной информации мы почти никогда не сможем однозначно интерпретировать события, происходящие в фазовом пространстве. Мы приведем здесь описание наиболее типичных ситуаций, которые следует иметь в виду при работе с конкретными примерами, а затем прокомментируем менее типичные сценарии.

1. $\operatorname{Eсли~}(h, f) \in \Phi(\mathscr{M}) \backslash \Sigma, m o$ $\mathscr{M}_{h, f}$ - один или несколько торов Лиувилля, описываемых теоремой 1 , кроме того,

- на каждом из торов в зависимости от соотношения частот $\omega_{1}, \omega_{2}$ траектории системы (1) задают периодические $\left(\omega_{1} / \omega_{2}-\right.$ рациональное), либо квазипериодические $\left(\omega_{1} / \omega_{2}-\right.$ иррациональное) обмотки. 
2. $\operatorname{Ecлu~}(h, f) \in \Sigma_{1}, m o$

$\mathscr{M}_{h, f}$ - набор замкнутых кривых $\gamma_{i}$ и особых двумерных многообразий, примыкающих к (неустойчивым) периодическим решениям, кроме того,

- всякая замкнутая кривая $\gamma_{i}$ либо соответствует периодическим решениям, либо состоит из неподвижных точек системы (1) (кривая состоит из неподвижных точек тогда и только тогда, когда касательная к кривой $\Sigma_{1}$ в точке $(h, f)$ параллельна прямой $h=$ const);

- всякое особое двумерное многообразие заполнено либо траекториями асимптотическими к соответствующему периодическому решению, либо периодическими траекториями.

3. $\operatorname{Ecлu~}(h, f) \in \Sigma_{0}, m o$

$\mathscr{M}_{h, f}$ - набор точек $P_{i}$ и особых двумерных или одномерных многообразий, примыкающих к неустойчивым неподвижным точкам, причем

- всякая точка $P_{i}$ является неподвижной точкой системы (1);

- особые двумерные многообразия заполнены решениями, асимптотическими к соответствующим неподвижным точкам, либо периодическими траекториями.

Имея в виду это описание, при анализе системы следует помнить о следующих возможностях.

- Даже если точка $(h, f)$ попала на бифуркационное множество $\Sigma$, вполне может случиться так, что одна из связных компонент интегрального многообразия $\mathscr{M}_{h, f}$ является обычным тором Лиувилля и никаких критических точек не содержит. Такой случай возникает, например, для волчка Горячева-Чаплыгина, который мы обсуждаем ниже. Избежать подобных неудобств помогает бифуркационный комплекс, являющийся естественным обобщением бифуркационной диаграммы (см. раздел 4).

- В случае $(h, f) \in \Sigma_{1}$ особое интегральное многообразие $\mathscr{M}_{h, f}$ может содержать двумерные куски, целиком состоящие из критических траекторий. Эти траектории могут быть как замкнутыми, так и незамкнутыми. В динамике твердого тела встречаются, например, ситуации, когда $\mathscr{M}_{h, f}$ представляет собой гладкий двумерный тор. С точки зрения динамики этот тор ничем не отличается от торов Лиувилля, но интегралы $H$ и $F$ на нем зависимы, поэтому тор отображается на бифуркационное множество.

- В случае $(h, f) \in \Sigma_{0}$ особое интегральное многообразие $\mathscr{M}_{h, f}$ вместе с точками ранга нуль может содержать замкнутые критические траектории, на которых ранг отображения момента равен единице (такая ситуация описана, например, в [29]). В результате траектории, лежащие на двумерных особых многообразиях, могут оказаться асимптотическими по отношению к таким критическим траекториям (а не к точкам $P_{i}$, как в стандартной ситуации).

2.2. Критические и некритические периодические траектории. Периодические траектории интегрируемых систем разделяются на два класса, для которых анализ устойчивости существенно различается.

ОПРЕДЕЛЕНИЕ. Замкнутая траектория интегрируемой гамильтоновой системы называется критической, если она целиком принадлежит множеству критических точек $S$ интегрального отображения $\Phi$. В противном случае она называется некритической. 
Напомним, что некритическая траектория не пересекается с критическим множеством вообще. Это сразу следует из того, что критическое множество инвариантно относительно гамильтонова потока.

Некритические замкнутые траектории бывают двух типов.

1. K первому типу (таких траекторий большинство) относятся те, которые лежат на инвариантных лиувиллевых торах. Эти торы автоматически будут резонансными, т. е. будут целиком состоять из замкнутых траекторий.

2. Второй тип (в некотором смысле исключительный) образуют замкнутые траектории, которые лежат на особых интегральных многообразиях $\left\{H=h_{0}, F=f_{0}\right\},\left(h_{0}, f_{0}\right) \in \Sigma$, но сами критическими не являются.

Пример ситуации, в которой могут появиться некритические периодические решения (второго типа), - это стандартная бифуркация, при которой один тор Лиувилля распадается на два. В момент бифуркации интегральное многообразие $\mathscr{M}_{h_{0}, f_{0}}$ становится прямым произведением восьмерки на окружность (рис. 3, a)). Оно, как нетрудно видеть, состоит из трех частей - замкнутой траектории и примыкающих к ней двух двумерных кусков (рис. 3, b)). Дифференциалы гамильтониана и интеграла становятся зависимыми в точности на этой замкнутой траектории $\gamma$, двумерные же куски критических точек интегрального отображения не содержат. В ситуации общего положения они целиком состоят из траекторий, асимптотических по отношению к этой замкнутой траектории (рис. 3, с)). Однако, в исключительных случаях вполне может случиться так, что эти траектории окажутся не асимптотическими, а замкнутыми (рис. 3, d)). Именно о подобных ситуациях мы здесь и говорим. Примером может служить случай Горячева-Чаплыгина в динамике твердого тела (см. п. 2.4).

Говоря о некритических замкнутых траекториях, полезно иметь в виду следующее наблюдение. Как мы уже отметили выше, замкнутость лишь одной траектории на торе Лиувилля гарантирует замкнутость всех траекторий, лежащих на этом торе. То же самое верно не только для торов, но и для особых интегральных многообразий. А именно, имеет место следующее утверждение.

ПреДЛОЖЕниЕ 1. Пусть $\mathscr{M}_{h_{0}, f_{0}}=\left\{H=h_{0}, F=f_{0}\right\}$ - связное интегральное многообразие (особое или неособое), содержащее хотя бы одну замкнутую некритическую траекторию. Тогда все некритические траектории, лежащие на этом многообразии, замкнуты.

ДокАЗАТЕЛЬСтво. Мы дадим доказательство, основанное на следующем простом факте, который интересен сам по себе: если на интегральной поверхности $\mathscr{M}_{h_{0}, f_{0}}$ имеется замкнутая некритическая траектория $\gamma$, то в некоторой окрестности $\mathscr{M}_{h_{0}, f_{0}}$ корректно определена переменная действия $I_{\gamma}$, которая гладко зависит от $H$ и $F$, причем все траектории соответствующего гамильтонова векторного поля $X_{I_{\gamma}}$ замкнуты и одна из них совпадает с $\gamma$. Другими словами, по крайней мере одна из переменных действия выживает и остается гладкой (аналитической), даже если данная интегральная поверхность имеет особенности [55]. 


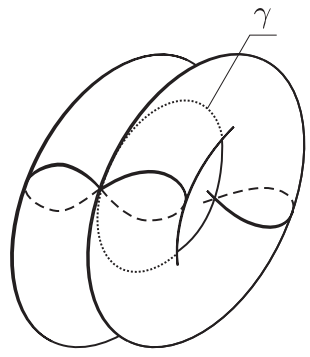

a)

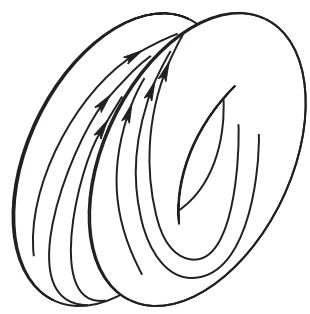

c)

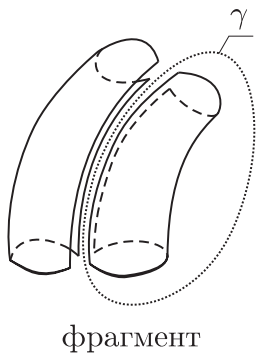

b)

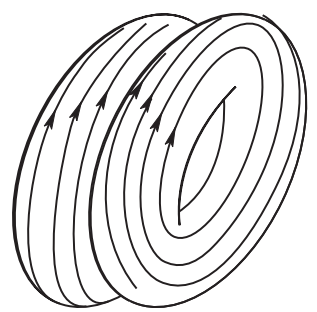

d)

Рис. 3. Возможное поведение траекторий на особом многообразии, примыкающем к периодической траектории

Существование такой переменной действия следует из того, что она может быть задана простой стандартной явной формулой, а именно:

$$
I_{\gamma}(h, f)=\frac{1}{2} \int_{\gamma(h, f)} \alpha,
$$

где $\alpha=p d q$ - форма действия, т. е. $d \alpha=\Omega$. При этом интегрирование ведется по замкнутым циклам $\gamma(h, f)$, которые получены из траектории $\gamma$ путем ее смещения на близкие интегральные поверхности $\mathscr{M}_{h, f}$ с заданными значениями гамильтониана и интеграла. Поскольку функции $H$ и $F$ независимы в окрестности траектории $\gamma$, то такая операция вполне законна и дает для каждой пары $(h, f)$, достаточно близкой к $\left(h_{0}, f_{0}\right)$, замкнутый цикл $\gamma(h, f) \subset \mathscr{M}_{h, f}$, однозначно определенный с точностью до гомотопии.

Ясно, что $I_{\gamma}$ - обычная переменная действия всюду за исключением особых интегральных многообразий. На особых интегральных многообразиях она, однако, остается гладкой и поэтому задаваемое ей гамильтоново векторное поле будет иметь замкнутые траектории всюду, где эта функция определена, в том числе и на особых интегральных многообразиях. Из построения также следует, что исходная траектория $\gamma$ будет одной из траекторий гамильтонова векторного поля $X_{I_{\gamma}}$. Используя тот факт, что $I_{\gamma}$ является функцией от $h$ и $f$, мы, кроме того, имеем

$$
X_{I_{\gamma}}=\frac{\partial I_{\gamma}}{\partial h} X_{H}+\frac{\partial I_{\gamma}}{\partial f} X_{F}
$$


Поскольку $\gamma$ - общая траектория для $X_{I_{\gamma}}$ и $X_{H}$, то мы заключаем, что $\frac{\partial I_{\gamma}}{\partial f}=0$ при $H=h_{0}, F=f_{0}$, т. е. всюду на интегральной поверхности $\mathscr{M}_{h_{0}, f_{0}}$ векторные поля $X_{I_{\gamma}}$ и $X_{H}$ пропорциональны. В частности, на $\mathscr{M}_{h_{0}, f_{0}}$ траектории этих векторных полей совпадают. Таким образом, все траектории нашей гамильтоновой системы, попавшие на $\mathscr{M}_{h_{0}, f_{0}}$, замкнуты просто потому, что это верно для переменной действия $I_{\gamma}$. Предложение 1 доказано.

В только что рассмотренном примере (рис. 3) особое интегральное многообразие состоит из двух двумерных кусков и замкнутой траектории. Предложение 1 утверждает, что если на одном из этих двумерных кусков имеется замкнутая траектория, то все траектории будут замкнуты не только на этом куске (что неудивительно), но и на втором (что менее тривиально). Отметим, что этот феномен является следствием не столько аналитичности (в гладком случае оно также верно), сколько гамильтоновости.

2.3. Невырожденные периодические траектории. Поведение интегрируемой (конформно) гамильтоновой системы является стандартным не только в окрестности неособого интегрального многообразия (теорема Лиувилля), но также в окрестности периодической траектории при условии ее невырожденности.

Имеется два очень близких подхода к понятию невырожденности замкнутой траектории. Первый из них является более общим и основан на динамике системы в окрестности траектории. Второй принципиально использует существование дополнительного интеграла $F$, в некотором смысле игнорируя динамику, и основан на структуре особенностей отображения момента на рассматриваемой траектории. В ситуации общего положения эти подходы совпадают, но имеются исключения, которые мы ниже обсудим.

Невырожденность в смысле теории динамических систем. С точки зрения теории динамических систем, периодическая траектория $\gamma$ гамильтоновой системы называется невырожденной, если ее мультипликаторы отличны от единицы (см., например, [50]).

Напомним определение мультипликаторов заданного периодического решения $\gamma: \mathbf{x}_{0}(t)=\mathbf{x}_{0}(t+T)$ с периодом $T$. Полагая $\mathbf{x}=\mathbf{x}_{0}(t)+\delta \mathbf{x}$ и линеаризуя уравнения по $\delta \mathbf{x}$, получаем уравнения в вариациях

$$
(\delta \mathbf{x})^{\bullet}=\mathbf{A}(t) \delta \mathbf{x}, \quad \mathbf{A}(t)=\mathbf{A}(t+T) .
$$

Если $\boldsymbol{\Lambda}(t)$ - фундаментальная матрица этой системы, причем $\boldsymbol{\Lambda}(0)=\mathbf{E}$, то $\boldsymbol{\Lambda}(T)$ - матрица монодромии. Собственные числа $\boldsymbol{\Lambda}(T)$ не зависят от начала отсчета времени и среди них имеется пара единичных, два оставшихся собственных числа $\lambda_{1}, \lambda_{2}$ называются мультипликаторами периодического решения (здесь идет речь о двух степенях свободы, в общем случае мультипликаторов больше).

Возможно другое эквивалентное определение мультипликаторов. Рассмотрим двумерное изоэнергетическое сечение Пуанкаре $\mathscr{N}_{h}$ к замкнутой траектории $\gamma$ в произвольной точке $\mathbf{x}_{0} \in \gamma$, где $h$ - постоянная, фиксирующая изоэнергетический уровень. На этом сечении фазовый поток системы (1) определяет 
двумерное отображение Пуанкаре $\pi: \mathscr{N}_{h} \rightarrow \mathscr{N}_{h}$, для которого периодическому решению $\gamma$ соответствует неподвижная точка $\mathbf{x}_{0}$. Собственные значения дифференциала отображения Пуанкаре в этой точке называются мулътипликаторами $\gamma$.

Вследствие сохранения фазового объема мультипликаторы удовлетворяют соотношению $\lambda_{1} \lambda_{2}=1$. В зависимости от их значений различают два типа замкнутых орбит:

- эллиптические, если $\lambda_{1}, \lambda_{2}$ - комплексные, лежащие на единичной окружности,

- гиперболические, если $\lambda_{1}, \lambda_{2}$ - вещественные и отличные по модулю от единицы.

Еще раз подчеркнем, что это определение является совершенно общим и никакой интегрируемости не предполагает.

Рассмотрим теперь ситуацию, когда гамильтонова система допускает дополнительный интеграл. Прежде всего отметим, что невырожденная в указанном выше смысле периодическом траектория $\gamma$ обязана быть критической (легко видеть, что мультипликаторы некритической замкнутой траектории автоматически равны единице). Тем самым, дифференциалы гамильтониана $H$ и дополнительного интеграла $F$ становятся пропорциональными на $\gamma$ или, что то же самое, интеграл $F$, ограниченный на изоэнергетическое сечение Пуанкаре $\mathscr{N}_{h}$ к критической траектории $\gamma$, имеет особенность в точке $\mathbf{x}_{0}=\gamma \cap \mathscr{N}_{h}$.

Невырожденность в смысле теории особенностей. Если посмотреть на ситуацию с точки зрения теории особенностей, то наиболее естественным будет следующее определение невырожденности (см. [6], [14]).

Критическая замкнутая траектория $\gamma$ называется невырожденной (с точки зрения теории особенностей), если $\mathbf{x}_{0}$ - невырожденная критическая точка для функции $\left.F\right|_{\mathscr{N}_{h}}$ в смысле теории Морса, т. е. если гессиан функции $\left.F\right|_{\mathscr{N}_{h}}$ невырожден. Другими словами, невырожденность траектории в смысле теории особенностей означает ее невырожденность как критического подмногообразия функции $\left.F\right|_{\left\{H=h_{0}\right\}}$.

При этом траектория называется

- эллиптической, если гессиан является знакоопределенным (т. е. его собственные значения одного знака),

- гиперболической в противном случае (собственные значения разных знаков $)^{1}$.

Два подхода к определению невырожденности замкнутой траектории - с точки зрения динамики и с точки зрения теории особенностей - почти совпадают. Однако имеются исключения, о которых следует помнить.

Если траектория $\gamma$ невырождена в смысле теории особенностей, то она, как несложно видеть, будет невырожденной в смысле динамики для почти всех линейных комбинаций вида $\lambda H+\mu F$. Однако всегда существует такая линейная комбинация, мультипликаторы которой равны единице. Поскольку с точки зрения теории особенностей мы рассматриваем особенности отображения момента в целом, никак по существу за динамикой не следим, то вполне может

\footnotetext{
${ }^{1}$ Имеется еще один подход к определению типов траектории, предложенный Л. М. Лерманом и Я.Л. Уманским [29].
} 
случиться так, что эта выделенная линейная комбинация "случайно" совпадает с самим гамильтонианом $H$. В этом случае траектория $\gamma$ окажется вырожденной с точки зрения гамильтонова векторного поля $X_{H}$, хотя на особенностях отображения момента это, разумеется, никак не отразится.

В обратную сторону похожая ситуация: почти всегда из невырожденности в смысле динамики следует невырожденность в смысле теории особенностей, но возможны исключения, которые на самом деле очень естественны. Действительно, если нам дана динамическая система, об интегралах которой мы ничего априори не знаем, то какие-либо выводы о конкретном интеграле сделать затруднительно просто потому, что интегралы однозначно не определены. Вполне может случиться так, например, что имеющийся у нас интеграл $F$ вырожден, но локально в окрестности рассматриваемой траектории существует гладкий интеграл $\widehat{F}$ такой, что $F=\widehat{F}^{2}$, и уже по отношению к этому новому интегралу $\widehat{F}$ наша траектория оказывается невырожденной.

Можно, конечно, предположить, что из невырожденности в смысле динамики следует существование некоторого невырожденного интеграла (который может отличаться от априори заданного). Но такое предположение без дополнительных предположений о нерезонансности оказывается также неверным. Следующий пример иллюстрирует возможные неприятности.

Представим себе, что дифференциал отображения Пуанкаре является поворотом на $\pi / 2$, т. е. мультипликаторы равны $e^{ \pm \pi i / 2}$ (резонансный случай). Можем ли мы что-то сказать об интеграле этой системы (если, разумеется, уже известно, что какой-то интеграл, независимый с $H$, существует)? Без дополнительных предположений ответ на этот вопрос окажется затруднительным, поскольку существуют принципиально различные функции, которые сохраняются при повороте на $\pi / 2$. Таковой будет, конечно, функция вида $p^{2}+q^{2}$, которая соответствует невырожденной ситуации в смысле теории особенностей, но имеются и другие, например, $\operatorname{Re}(p+i q)^{4}=p^{4}-6 p^{2} q^{2}+q^{4}$. Если у нашей системы интегралом оказалась последняя, то никакими средствами (типа извлечения квадратного корня как выше) мы не сумеем сделать особенность морсовской, и траектория будет вырожденной для любого интеграла.

Для целей настоящей работы подход, основанный на теории особенностей, оказывается более удобным и эффективным. Это совсем неудивительно, поскольку использование имеющейся дополнительной информации (в данном случае дополнительного интеграла) должно заведомо упрощать анализ системы.

Траектории эллиптического типа. Итак, рассмотрим периодическую траекторию, невырожденную в смысле теории особенностей. В эллиптическом случае справедлив следующий результат, который полностью описывает поведение системы в окрестности такой траектории.

ТЕОРема 2. В окрестности невырожденной эллиптической траектории $\gamma$ существуют канонические координаты $I, \varphi, p, q(\{\varphi, I\}=\{q, p\}=1)$ такие, что

$$
H=H\left(I, p^{2}+q^{2}\right), \quad F=F\left(I, p^{2}+q^{2}\right) .
$$


При этом угловая координата $\varphi(\bmod 2 \pi)$ вдоль кривой $\gamma$ определена так, что отождествляются точки с координатами $(I, \varphi, p, q) u(I, \varphi+2 \pi, p, q)$, a исходная замкнутая траектория задается уравнением $p=q=0, I=0$.

Кроме того, выполнены условия

$$
\frac{\partial H}{\partial I} \neq 0, \quad \operatorname{det}\left(\begin{array}{ll}
\frac{\partial H}{\partial I} & \frac{\partial H}{\partial J} \\
\frac{\partial F}{\partial I} & \frac{\partial F}{\partial J}
\end{array}\right) \neq 0
$$

где $J=\left(p^{2}+q^{2}\right) / 2$. При этом мулътипликаторы траектории $\gamma$ имеют вид $\lambda_{1,2}=e^{ \pm i 2 \pi \alpha}$, гдe

$$
\alpha=\left.\left(\frac{\partial H}{\partial I}\right)^{-1} \frac{\partial H}{\partial J}\right|_{I=J=0} .
$$

Как следует из этой теоремы, в окрестности эллиптической орбиты движение описывается уравнениями

$$
\dot{I}=\dot{J}=0, \quad \dot{\varphi}=\frac{\partial H(I, J)}{\partial I}, \quad \dot{\psi}=\frac{\partial H}{\partial J}(I, J),
$$

где угол $\psi$ определяется уравнениями $q=\sqrt{2 J} \cos \psi, p=\sqrt{2 J} \sin \psi$. Другими словами, траектории системы представляют собой квазипериодические и периодические обмотки двумерных торов, определяемых соотношениями

$$
I=\text { const }, \quad J=\text { const. }
$$

Отметим также, что невырожденность и эллиптичность траектории $\gamma$ с точки зрения общей теории динамических систем в контексте этой теоремы просто означает, что $\frac{\partial H}{\partial J} \neq 0$.

ЗАмЕЧАниЕ. Эта и следующая теоремы в теории особенностей интегрируемых гамильтоновых систем являются частными случаями существенно более общих результатов работ [32], [33], [56], хотя в такой форме они нигде не сформулированы.

Траектории гиперболического типа. Гиперболические траектории системы могут быть двух топологически различных типов в зависимости от структуры особого уровня $\left\{F=f_{0}, H=h_{0}\right\}$ в ее окрестности. Ясно, что локально особый уровень представляет собой двумерное многообразие с особенностью вдоль этой критической траектории. На трансверсали $\mathscr{N}_{h_{0}}$ к траектории мы всегда имеем "крест" (заданный уравнением типа $x y=0$ ), составленный из сепаратрис траектории $\gamma$. Однако глобально, перемещая трансверсаль вдоль траектории и следя за поведением "креста", мы обнаружим две возможные ситуации: "крест" может вернуться назад в прежнее положение (в этом случае мультипликаторы положительны), а может повернуться на угол $\pi$ (тогда мультипликаторы отрицательны).

В первом случае особое интегральное многообразие $\mathscr{M}_{h_{0}, f_{0}}$ локально будет представлять собой прямое произведение креста на окружность, а во втором косое произведение (его можно представлять себе как пару листов Мёбиуса, 
трансверсально пересекающихся по общей средней линии), соответствующие особые интегральные многообразия $\mathscr{M}_{h_{0}, f_{0}}$ см. рис. 1 и рис. 4 соответственно. Следуя А. Т. Фоменко [14], мы будем говорить, что траектория имеет ориентируемую сепаратрисную диаграмму в первом случае и, соответственно, неориентируемую - во втором.

Поведение системы в этом случае описывается следующим образом.

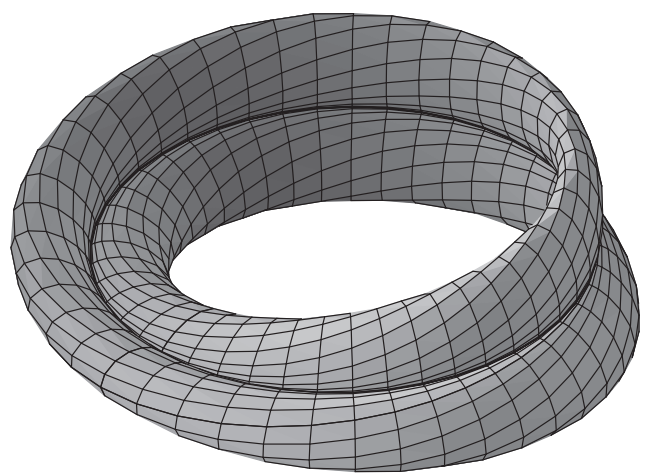

Рис. 4. Особый слой, содержащий гиперболическую траекторию с неориентируемой сепаратрисной диаграммой

ТеОрема 3. В окрестности невырожденной гиперболической траектории $\gamma$ существуют канонические координаты $I, \varphi, p, q(\{\varphi, I\}=\{q, p\}=1)$ такие, что

$$
H=H(I, p q), \quad F=F(I, p q) .
$$

При этом угловая координата $\varphi(\bmod 2 \pi)$ вдоль кривой $\gamma$ определена так, что

- в случае ориентируемой сепаратрисной диаграммы отождествляются точки с координатами $(I, \varphi, p, q)$ u $(I, \varphi+2 \pi, p, q)$,

- в случае неориентируемой - точки $(I, \varphi, p, q) u(I, \varphi+2 \pi,-p,-q)$, а исходная траектория $\gamma$ определена уравнениями $I=0, p=q=0$.

Кроме того, выполнены соотношения

$$
\frac{\partial H}{\partial I} \neq 0, \quad \operatorname{det}\left(\begin{array}{ll}
\frac{\partial H}{\partial I} & \frac{\partial H}{\partial J} \\
\frac{\partial F}{\partial I} & \frac{\partial F}{\partial J}
\end{array}\right) \neq 0,
$$

где $J=p q$. При этом мультипликаторы траектории $\gamma$ имеют следующий вид:

- в случае ориентируемой диаграммы $\lambda_{1,2}=e^{ \pm 2 \pi \alpha}$,

- в случае неориентируемой $\lambda_{1,2}=-e^{ \pm 2 \pi \alpha}$, где $\alpha=\left.\left(\frac{\partial H}{\partial I}\right)^{-1} \frac{\partial H}{\partial J}\right|_{I=J=0}$.

В неинтегрируемом случае для периодических траекторий гиперболического типа справедлива теорема Гробмана-Хартмана, аналогичная теореме 3 , при этом в окрестности траектории существует интеграл $F=p q$ [57]. 
Две приведенные теоремы полностью описывают возможную динамику системы в окрестности невырожденной траектории и структуру отображения момента. В частности, очевидно, что эллиптические критические траектории орбитально устойчивы, а гиперболические - неустойчивы. Помимо этого теоремы 2 и 3 имеют и другие важные следствия (которые, впрочем, можно легко получить и независимым путем).

СЛЕДСТВИЕ 1. Каждая критическая невырожденная траектория $\gamma$ включается в однопараметрическое семейство $\gamma_{h}$ невырожденных траекторий того же типа, причем в качестве параметра можно взять значение гамильтониана.

$B$ иелом траектории этого семейства образуют двумерное инвариантное симплектическое подмногообразие $P=\left\{\gamma_{h} \mid h_{0}-\varepsilon<h<h_{0}+\varepsilon\right\}$, которое целиком состоит из критических точек интегрального отображения $\Phi$.

Образом этого семейства при интегральном отображении является гладкая кривая (одна из ветвей бифуркачионной диаграммы), которая на плоскости $\mathbb{R}^{2}(h, f)$ может быть задана как график некоторой гладкой функции $f=f(h)$.

Производная $f_{h}^{\prime}$ при этом имеет следующий естественный смысл: она равна коэффициенту пропорциональности между гамильтоновыми векторными полями $X_{H}$ и $X_{F}$ на соответствующей траектории $\gamma_{h}$ из рассматриваемого семейства, m.e.

$$
f_{h}^{\prime}=\lambda, \quad \text { əде } X_{F}=\lambda X_{H}
$$

При этом в достаточно малой окрестности исходной траектории $\gamma=\gamma_{h_{0}}$ никаких других особъх точек интегрального отображения нет (другими словами, по крайней мере локально семейство $\left\{\gamma_{h}\right\}$ является изолированным и никак не пересекается с другими критическими траекториями или положениями равновесия).

Таким образом, невырожденные траектории (по крайней мере локально) образуют изолированные однопараметрические семейства, являющиеся инвариантными симплектическими подмногообразиями системы, которые при интегральном отображении переходят в гладкие кривые бифуркационной диаграммы.

Это утверждение локальное. В то же время при анализе системы важно понимать, что происходит с этими семействами в целом. Наиболее типичные сценарии таковы:

- Эллиптические невырожденные траектории могут сменить свой тип и превратиться в гиперболические. Само однопараметрическое семейство критических траекторий при этом остается гладким, но в момент перехода происходят три события: 1) траектория перестает быть невырожденной; 2) многообразие, состоящее из траекторий, перестает быть симплектическим; 3) на бифуркационной диаграмме возникает касп (см., например, [29], [58]).

- Несколько семейств невырожденных траекторий взаимодействуют между собой. Например, из эллиптической траектории могут возникнуть две 
эллиптические (или одна, но с вдвое большим периодом) и одна гиперболическая (бифуркация "вилки" и бифуркация "удвоения периода"). На бифуркационной диаграмме мы в этом случае увидим несколько (обычно две) ветви бифуркационной диаграммы, сходящиеся в одной. Обычно в такой ситуации ветви имеют общую касательную и тангенс угла ее наклона, как и в невырожденном случае, совпадает с коэффициентом пропорциональности гамильтоновых полей $X_{H}$ и $X_{F}$ на той (разумеется, вырожденной!) периодической траектории, где сходятся вместе несколько семейств (см., например, [22], [59]).

- Наконец, семейство невырожденных траекторий может взаимодействовать с положениями равновесия (т. е. с такими точками, где ранг отображения момента равен нулю). Обычно при таких сценариях пересекаются два симплектических многообразия $P_{1}$ и $P_{2}$, составленных из невырожденных замкнутых траекторий (бифуркации типа центр-центр, центрседло, седло-седло). Сами траектории в момент бифуркации либо превращаются в неподвижную точку (случай центра), либо перестают быть замкнутыми, а становятся асимптотическими по отношению к некоторому положению равновесия. Симплектичность многообразий $P_{1}$ и $P_{2}$ при этом не теряется, они пересекаются трансверсально в неподвижной точке системы, а на бифуркационной диаграмме мы увидим две трансверсально пересекающиеся ветви (одна или обе из них могут закончиться в точке контакта), см., например, [14], [29], [55].

Этот список не исчерпывает всех априори возможных случаев. Например, в случае Горячева-Чаплыгина несколько семейств (три или даже четыре) могут сходиться вместе, но не к вырожденной траектории, а к вырожденному положению равновесия.

2.4. Бифуркационная диаграмма случая Горячева-Чаплыгина. Для описания критического множества и построения бифуркационной диаграммы волчка Горячева-Чаплыгина (5) воспользуемся представлением этой системы в разделяющихся переменных [4], [51]. Соответствующие канонические переменные на симплектическом листе нулевой постоянной площадей $\mathscr{M}_{0}=\left\{\mathbf{M}, \boldsymbol{\gamma} \mid \boldsymbol{\gamma}^{2}=1,(\mathbf{M}, \boldsymbol{\gamma})=0\right\}$ определяются соотношениями

$$
\begin{gathered}
M_{1}=2 \sqrt{p_{1} p_{2}} \sin \frac{q_{1}-q_{2}}{2}, \quad M_{2}=2 \sqrt{p_{1} p_{2}} \cos \frac{q_{1}-q_{2}}{2}, \quad M_{3}=p_{1}-p_{2}, \\
\gamma_{1}=\frac{p_{1} \sin q_{1}+p_{2} \sin q_{2}}{p_{1}+p_{2}}, \quad \gamma_{2}=\frac{p_{1} \cos q_{1}+p_{2} \cos q_{2}}{p_{1}+p_{2}} \\
\gamma_{3}=-\frac{2 \sqrt{p_{1} p_{2}}}{p_{1}+p_{2}} \cos \frac{q_{1}+q_{2}}{2}
\end{gathered}
$$

где $q_{1}, q_{2} \in[0,2 \pi)$ - угловые переменные, а $p_{1}, p_{2}>0$ - соответствующие импульсы (при этом $\left.\left(p_{1}+p_{2}\right)^{2}=(\mathbf{M}, \mathbf{M}) \neq 0\right)$. Следовательно, переменные $p_{i}$ и $q_{i}(\bmod 2 \pi)$ определены на полуцилиндре, причем особенность, которую замена (8) имеет на окружности $p_{i}=0$, аналогична особенности перехода к полярным координатам вида $x=\sqrt{2 \rho} \cos \varphi, y=\sqrt{2 \rho} \sin \varphi$ при $\rho=0$ (т. е. в начале координат). Следовательно, при замене (8) все точки на окружности $p_{i}=0$ 
также должны быть отождествлены между собой (т. е. эта окружность склеивается в одну точку).

Гамильтониан и первый интеграл (5) представляются в виде

$$
\begin{aligned}
& H=\frac{2}{p_{1}+p_{2}}\left(p_{1}^{3}+p_{2}^{3}-\frac{\mu}{2}\left(p_{1} \sin q_{1}+p_{2} \sin q_{2}\right)\right), \\
& F=\frac{4 p_{1} p_{2}}{p_{1}+p_{2}}\left(p_{1}^{2}-p_{2}^{2}-\frac{\mu}{2}\left(\sin q_{1}-\sin q_{2}\right)\right)
\end{aligned}
$$

при этом несложно показать, что совместный уровень функций $\mathscr{M}_{h, f}=\{\mathbf{M}, \boldsymbol{\gamma} \mid$ $H=h, F=f\}$ описывается разделенными уравнениями:

$$
\begin{aligned}
& \varphi_{1}\left(q_{1}, p_{1}\right)=p_{1}\left(p_{1}^{2}-\frac{\mu}{2} \sin q_{1}-\frac{h}{2}\right)-\frac{f}{4}=0, \\
& \varphi_{2}\left(q_{2}, p_{2}\right)=p_{2}\left(p_{2}^{2}-\frac{\mu}{2} \sin q_{2}-\frac{h}{2}\right)+\frac{f}{4}=0 .
\end{aligned}
$$

ЗАмЕчАниЕ. Приведение уравнений движения на поверхности уровня $\mathscr{M}_{h, f}$ к форме уравнений Абеля-Якоби было выполнено С. А. Чаплыгиным [60] явно с помощью переменных $p_{1}, p_{2}$. Построение полного канонического набора разделяющих переменных и их связь с переменными Андуайе были описаны В.В. Козловым [61]. Различные обобщения интегрируемого случая ГорячеваЧаплыгина при помощи этих переменных получены в работах [62], [63].

Представление (10) естественным образом позволяет описать слоение на торы Лиувилля рассматриваемой системы. Заметим, что уравнения (10) инвариантны относительно замены $f \rightarrow-f, \varphi_{1} \rightarrow \varphi_{2}, \varphi_{2} \rightarrow \varphi_{1}$, поэтому достаточно рассмотреть случай $f>0$. Построим линии уровня функции

$$
\bar{\varphi}(p, q)=p\left(p^{2}-\frac{\mu}{2} \sin q-\frac{h}{2}\right),
$$

они замкнуты (на цилиндре) и имеют качественно различный вид при $-\mu<$ $h<\mu$ и $h>\mu$ (см. рис. 5).

Как следует из (10), всякой возможной паре кривых $\bar{\varphi}=f, \bar{\varphi}=-f$ соответствует тор Лиувилля. Из рис. 5 видно, что при $-\mu<h<\mu$ всякому возможному значению $f$ соответствует одна пара кривых и соответственно один инвариантный тор. При $h>\mu$ для значений $c_{*}<\bar{\varphi}<0$, соответствующих на рисунке выжеленной серым цветом области, имеется пара кривых и, следовательно, при данных значениях интегралов $(h, f)$ имеются две пары инвариантных торов.

Исключение составляет кривая $\bar{\varphi}=0$, так как при $h>\mu$ (см. рис. $5, \mathrm{~b}))$ ей соответствует инвариантный тор, который, как можно показать на основании квадратур с использованием (10), является резонансным (т. е. целиком заполнен замкнутыми траекториями).

При $-\mu<h<\mu$ кривой $\bar{\varphi}=0$ соответствует особый слой, представляющий собой перекрученное произведение восьмерки на окружность, см. рис. 4 (подробное описание содержится в книге [13]), который также является резонансным и заполнен периодическими траекториями. Характерный вид движений тела в этих случаях приведен в книге [51]. 


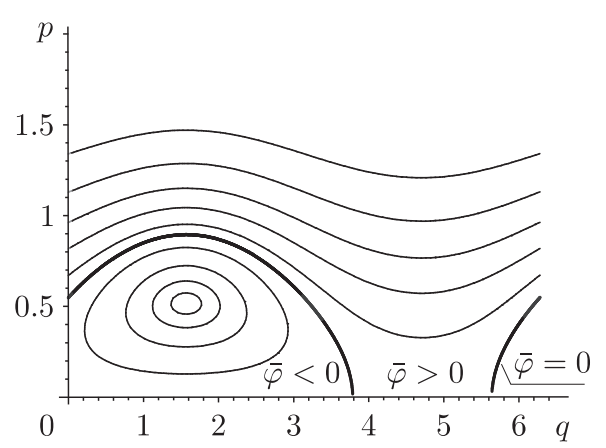

a) $-\mu<h<\mu$

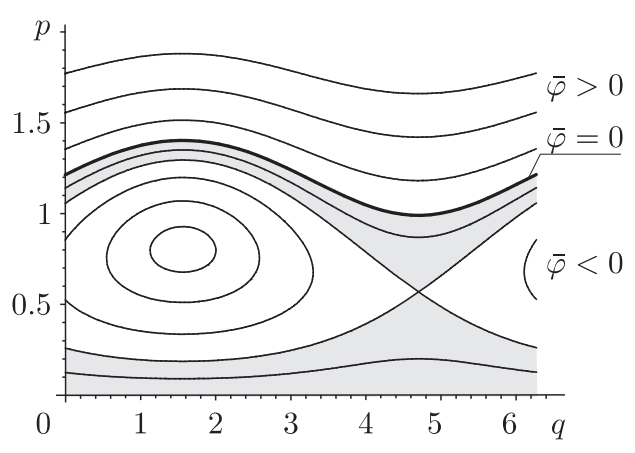

b) $h>\mu$

Рис. 5. Линии уровня функции $\bar{\varphi}(p, q)$. Параметры: $\mu=1$, а) $h=0.6$, b) $h=3$.

Очевидно, что условия падения ранга отображения $\Phi$ эквивалентны условиям вырождения кривых, описываемых уравнениями (10). Это позволяет достаточно просто описать критическое множество и построить бифуркационную диаграмму данной системы.

Множество $S_{1}$ и его образ $\Sigma_{1}$. Начнем с исследования условий вырождения кривой, описываемой соотношением $\varphi_{1}=0$. Соответствующее множество в фазовом пространстве определяется системой уравнений

$$
\frac{\partial \varphi_{1}}{\partial q_{1}}=\frac{\partial \varphi_{1}}{\partial p_{1}}=0, \quad \varphi_{1}=0, \quad \varphi_{2}=0 .
$$

Отсюда находим, что имеется три соответствующих семейства в $S_{1}$.

а) Одно семейство периодических орбит определено соотношениями

$$
p_{1}=0 \quad\left(p_{2} \neq 0\right), \quad f=0, \quad h=2\left(p_{2}^{2}-\frac{\mu}{2} \sin q_{2}\right)
$$

и параметризуется величиной $h>-\mu$, соответствующая кривая в $\Sigma_{1}$ задается уравнением

$$
f=0, \quad h>-\mu .
$$

b) Еще два семейства определены условиями

$$
\begin{gathered}
\cos q_{1}=0, \quad f=-8 p_{1}^{3}, \quad h= \pm \mu+6 p_{1}^{2}, \\
p_{2}\left(p_{2}^{2}-\frac{\mu}{2} \sin q_{2} \mp \frac{\mu}{2}-3 p_{1}^{2}\right)=4 p_{1}^{3},
\end{gathered}
$$

где одновременно берутся либо только верхние знаки, либо только нижние.

Обозначая $2 p_{1}=\lambda>0$, получим более привычное [14], [13], [51], представление соответствующих множеств $\Sigma_{1}$ :

$$
f=-\lambda^{3}, \quad h= \pm \mu+\frac{3}{2} \lambda^{2} .
$$




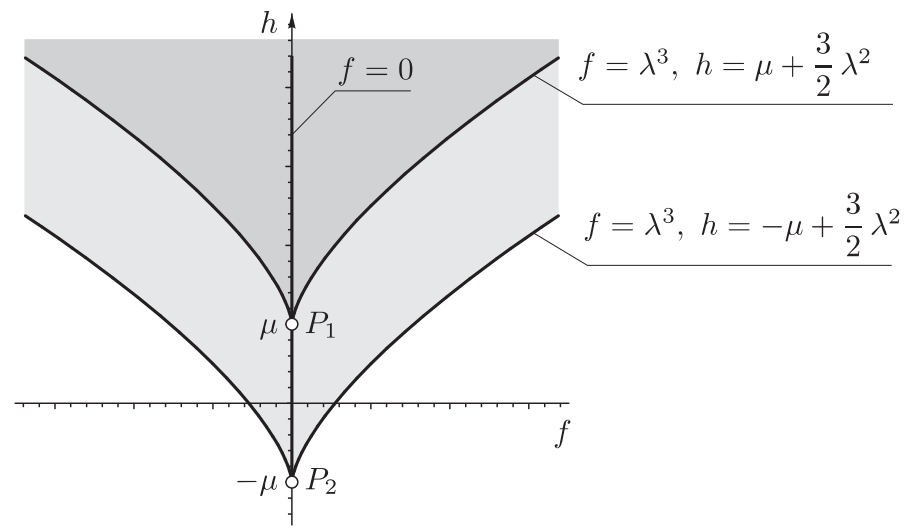

Рис. 6. Бифуркационная диаграмма случая Горячева-Чаплыгина. Более темным цветом отмечена область, в которой каждой точке соответствует пара инвариантных торов.

Аналогично записываются условия вырождения кривой $\varphi_{2}=0$ :

$$
\frac{\partial \varphi_{2}}{\partial q_{2}}=\frac{\partial \varphi_{2}}{\partial p_{2}}=0, \quad \varphi_{1}=0, \quad \varphi_{2}=0,
$$

откуда находим еще три семейства, входящих в критическое множество системы $S_{1}$.

$\mathrm{a}^{\prime}$ ) Семейство, определенное условиями

$$
p_{2}=0 \quad\left(p_{1} \neq 0\right), \quad f=0, \quad h=2\left(p_{1}^{2}-\frac{\mu}{2} \sin q_{1}\right) .
$$

Так же, как и выше, ему соответствует бифуркационная кривая

$$
f=0, \quad h>-\mu .
$$

$\left.\mathrm{b}^{\prime}\right)$ Два других семейства определяются уравнениями

$$
\begin{gathered}
\cos q_{2}=0, \quad f=8 p_{2}^{3}, \quad h= \pm \mu+6 p_{2}^{2}, \\
p_{1}\left(p_{1}^{2}-\frac{\mu}{2} \sin q_{1} \mp \mu-3 p_{2}^{2}\right)=4 p_{2}^{3},
\end{gathered}
$$

где нужно брать либо только верхние, либо только нижние знаки.

Обозначая, как и выше, $2 p_{2}=\lambda$, получим привычную параметрическую форму для бифуркационных кривых:

$$
f=\lambda^{3}, \quad h= \pm \mu+\frac{3}{2} \lambda^{2} .
$$

При $-\mu<h<\mu$ формулы (11), (14) определяют одно и то же решение маятникового типа, при котором центр масс колеблется в неподвижной плоскости: соотношение (11) описывает одну половину колебания в одну сторону, а (14) вторую половину в обратную. 
При $h>\mu$ соотношение (11) и соотношение (14) описывают два различных решения - вращения центра масс в неподвижной плоскости.

Множество $S_{0}$ и его образ $\Sigma_{0}$. Условие одновременного вырождения функций (10) приводит к соотношениям

$$
p_{1}=0, \quad p_{2}=0
$$

которые обозначают, что соответствующие значения канонических переменных (8) выходят за область их определения. Тем не менее несложно проанализировать условие $\operatorname{rank} \mathrm{d} \Phi=0$ в исходных переменных $\mathbf{M}, \boldsymbol{\gamma}$, откуда получим, что $S_{0}$ состоит из пары неподвижных точек, определяемых соотношениями

$$
M_{1}=M_{2}=M_{3}=0, \quad \gamma_{2}=\gamma_{3}=0, \quad \gamma_{1}= \pm 1 .
$$

При этом $\Sigma_{0}$ также состоит из пары точек $P_{1}, P_{2}$, определяемых уравнениями

$$
f=0, \quad h= \pm \mu .
$$

ЗАмЕчаниЕ. В статье [64] анализ особых периодических решений, лежащих на бифуркационных кривых, был дополнен некоторыми полезными с точки зрения механики соображениями, связанными с визуализацией движения в абсолютном пространстве. Этот метод позволяет из периодических решений в приведенном фазовом пространстве выделить такие, которые соответствуют периодическим решениям в абсолютном фазовом пространстве, именно они представляют наибольший интерес для динамики. Некоторые из таких решений особо замечательны тем, что помимо всего они являются устойчивыми. В [64] дан анализ периодических решений в фазовом пространстве для интегрируемых случаев уравнений Эйлера-Пуассона. Аналоги таких решений в небесной механике называются хореографиями [65], [66]. В дальнейшем эта идея была развита нами (при помощи метода продолжения по параметру и построения генеалогии периодических решений) для анализа периодических решений неинтегрируемых систем [67].

2.5. Бифуркационная диаграмма случая Клебша. В случае Клебша имеются два отличия от случая Горячева-Чаплыгина, которые существенно усложняют описание критического множества и построение бифуркационной диаграммы. Во-первых, система Клебша интегрируема при произвольной постоянной площадей $c=(\mathbf{M}, \boldsymbol{\gamma})$, так что при изменении этого параметра меняется структура критического множества и вид бифуркационных диаграмм. Во-вторых, не известна система вещественных разделяющих переменных.

ЗАмЕчАниЕ. Вопросы, связанные с поиском разделяющих переменных и явным интегрированием системы Клебша, обсуждаются в систематической форме в сборнике [68]. Исследованием задачи Клебша и изоморфных ей систем занимались многие выдающиеся математики и механики, такие как С. А. Чаплыгин, А. М. Ляпунов, В.А. Стеклов, Г. Минковский, Ф. Шоттки, В. Фрам, Г. Колосов и др. Отметим, что вещественного разделения переменных для этого случая интегрируемости так и не было получено. В литературе встречаются ссылки на работу Ф. Кеттера [69], где система Клебша была проинтегрирована 
в тета-функциях. Тем не менее это решение Кеттера носит чисто комплексный характер и не может быть использовано для качественного анализа поведения системы. Реальной информации о динамике системы Клебша нельзя извлечь и из более современных работ (см., например, [70]).

Конечно, нахождение явного разделения переменных существенно облегчает топологический анализ динамической системы. Однако эффективное топологическое исследование возможно и в тех случаях, когда разделение переменных неизвестно. Отметим, что именно задача Клебша и послужила толчком для разработки топологических методов, не опирающихся на разделение переменных. Идея такого анализа была предложена и развита в работах [8]-[12], где были построены все возможные бифуркационные диаграммы и области возможных движений задачи Клебша. В дальнейшем эти методы получили широкое развитие и были применены для анализа большинства известных классических задач динамики твердого тела [14]-[17], [19], [20].

Отметим, что даже в случае систем с известным разделением переменных оказывается необязательным использовать дальнейшее явное интегрирование, например, в тета-функциях. По крайней мере, при анализе частных решений и их устойчивости мы никак не пользовались их явным представлением через тета-функции (даже в случае Горячева-Чаплыгина). По сравнению с топологическим подходом, использование аппарата тета-функций для анализа поведения системы существенно более трудная задача.

Детальный анализ критических значений $h, f$ и бифуркационных диаграмм выполнен в этом случае Т. И. Погосяном [9]. Он использовал представление системы Клебша в локальных канонических координатах на $\mathscr{M}_{c}$ (сферические координаты и сопряженные им импульсы). Здесь мы воспользуемся другим подходом, давая описание критического множества в исходных (избыточных) фазовых переменных $\mathbf{M}, \gamma$.

Критическое множество $S_{1}$. Как известно из теории функций многих переменных, условие падения ранга интегрального отображения $\Phi$ на фиксированном симплектическом листе $\mathscr{M}_{c}$ эквивалентно требованию линейной зависимости градиентов всех четырех функций $H, F, K_{1}, K_{2}$ при фиксированных значениях казимиров $K_{1}=1, K_{2}=c$. С помощью неопределенных множителей Лагранжа эта задача сводится к системе уравнений вида

$$
\begin{gathered}
\frac{1}{2} \frac{\partial F}{\partial \mathbf{x}}=\lambda \frac{\partial H}{\partial \mathbf{x}}-\frac{1}{2} \eta \frac{\partial K_{1}}{\partial \mathbf{x}}-\xi \frac{\partial K_{2}}{\partial \mathbf{x}}, \\
K_{1}=1, \quad K_{2}=c,
\end{gathered}
$$

где неизвестными являются $\lambda, \eta, \xi, \mathbf{x}=\{\mathbf{M}, \gamma\}$.

Из первых шести уравнений этой системы находим

$$
\begin{gathered}
\mathbf{M}=\eta \mathbf{I}(\lambda \mathbf{E}-\mathbf{I})^{-1} \boldsymbol{\gamma}, \\
\eta^{2}=\frac{\mu}{\lambda} \operatorname{det}(\lambda \mathbf{E}-\mathbf{I}), \quad \xi=\frac{\mu \operatorname{det} \mathbf{I}}{\lambda}\left(\lambda \operatorname{Tr} \mathbf{I}^{-1}-1\right),
\end{gathered}
$$

где $\mathbf{I}=\mathbf{A}^{-1}$.

Подставляя (16) в оставшиеся два уравнения, получим систему

$$
(\boldsymbol{\gamma}, \boldsymbol{\gamma})=1, \quad \eta\left(\mathbf{I}(\lambda \mathbf{E}-\mathbf{I})^{-1} \gamma, \gamma\right)=c,
$$


которая (при $c \neq 0$ ) описывает при фиксированном $\lambda$ пересечение сферы с эллипсоидом и (в случае существования решений) определяет пару замкнутых кривых. Таким образом, в данном случае получаем представление критического множества в виде семейства кривых, параметризуемых величиной $\lambda$, которые задаются уравнениями (16), (17). Из условия положительной определенности величины $\eta^{2}$ получим три интервала возможных значений параметра:

$$
\lambda \in(-\infty, 0) \cup\left(I_{1}, I_{2}\right) \cup\left(I_{3},+\infty\right) .
$$

ЗАмЕчАниЕ. Эти периодические траектории можно также найти исходя из условий существования инвариантных многообразий, задаваемых соотношениями

$$
M_{i}=\Lambda_{i} \gamma_{i}, \quad \Lambda_{i}=\text { const. }
$$

Этим способом они были найдены В.А. Стекловым [71], который также дал несложное сведение к квадратурам. Кстати, этот результат Стеклова (который приведен в книге [51]) был недавно отмечен в [45] в качестве нового частного решения. Правда, кинематическая и динамическая интерпретация этих решений до сих пор не получена.

Образ этого критического множества $\Sigma_{1}$ на плоскости $h, f$ можно получить, выражая какую-либо пару величин, например $\gamma_{1}^{2}, \gamma_{2}^{2}$, из уравнений (17) и подставляя в уравнения для интегралов (6). С учетом возможного извлечения корней для $\eta$ с различными знаками находим

$$
\begin{aligned}
h_{ \pm} & =\frac{\mu}{2 \lambda^{2}}\left(\operatorname{det} \mathbf{I} \pm \frac{c}{\eta}\left(2 \lambda^{3}-\lambda^{2} \operatorname{Tr} \mathbf{I}+\operatorname{det} \mathbf{I}\right)\right), \\
f_{ \pm} & =\frac{\mu}{\lambda}\left(\left(2-\lambda \operatorname{Tr} \mathbf{I}^{-1}\right) \operatorname{det} \mathbf{I} \pm \frac{c}{\eta}\left(\lambda^{2} \operatorname{Tr} \mathbf{I}-2 \lambda \operatorname{Tr} \mathbf{I}^{-1} \operatorname{det} \mathbf{I}+3 \operatorname{det} \mathbf{I}\right)\right),
\end{aligned}
$$

где для $\eta$ фиксировано положительное значение корня.

Выбор знаков в (18) и значений параметра $\lambda$, соответствующих физически допустимым движениям (т. е. условий разрешимости уравнений $(16),(17)$ при фиксированных $h, f)$ в этом случае приводит к простым, но довольно громоздким вычислениям. Ниже мы приведем ответ, основываясь на известных результатах работы [9] (полученных другим методом).

Кроме того, необходимо учесть, что в связи с инвариантностью интегралов (6) и соотношения (16) относительно замены $\mathbf{M}, \boldsymbol{\gamma} \rightarrow-\mathbf{M},-\boldsymbol{\gamma}$ каждому возможному значению параметра $\lambda$ соответствует два различных периодических решения. Действительно, как будет показано ниже, каждое семейство примыкает к неподвижным точкам, причем одним и тем же фиксированным значениям первых интегралов соответствует пара неподвижных точек в фазовом пространстве, следовательно, к каждой точке примыкает по семейству.

Критическое множество $S_{0}$. Условие $\operatorname{rank} \mathrm{d} \Phi=0$ в данном случае можно представить в форме системы уравнений

$$
\begin{array}{rlrl}
\frac{\partial F}{\partial \mathbf{x}}=\lambda_{1} \frac{\partial K_{1}}{\partial \mathbf{x}}+\lambda_{2} \frac{\partial K_{2}}{\partial \mathbf{x}}, & \frac{\partial H}{\partial \mathbf{x}} & =\lambda_{3} \frac{\partial K_{1}}{\partial \mathbf{x}}+\lambda_{4} \frac{\partial K_{2}}{\partial \mathbf{x}}, \\
K_{1}=1, & K_{2}=c,
\end{array}
$$




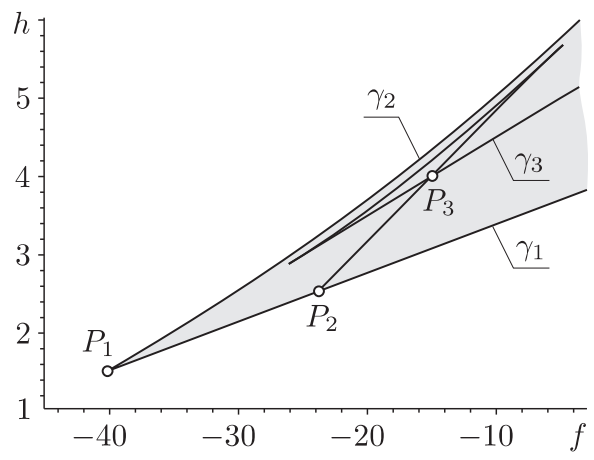

Рис. 7. Бифуркационная диаграмма при условии $\frac{c}{\sqrt{\mu \operatorname{det} \mathbf{I}}}<\sqrt{a_{1}-a_{3}}-$ $\sqrt{a_{2}-a_{3}}$. Для параметров зафиксированы следующие значения: $I_{1}=3$, $I_{2}=5, I_{3}=7, \mu=1, c=0.23$.

где неизвестными являются неопределенные множители $\lambda_{i}, i=1, \ldots, 4$, и фазовые переменные $\mathbf{x}=(\mathbf{M}, \boldsymbol{\gamma})$.

Система (19) на каждом листе $\mathscr{M}_{c}$ имеет шесть решений, определяющих $S_{0}$, которые представляются в виде

$$
M_{i}=M_{j}=0, \quad \gamma_{i}=\gamma_{j}=0, \quad M_{k}= \pm c, \quad \gamma_{k}= \pm 1,
$$

где берется циклическая перестановка $i j k$ и один и тот же знак. Этим решениям соответствуют три точки $P_{k} \in \Sigma_{0}, k=1,2,3$, определяемые равенствами

$$
h_{k}^{(0)}=\frac{1}{2}\left(\frac{c^{2}}{I_{k}}+\mu I_{k}\right), \quad f_{k}^{(0)}=c^{2}-\mu I_{i} I_{j},
$$

где также используется циклическая перестановка $i j k$.

Бифуркационная диаграмма. Упорядочим величины $I_{1}<I_{2}<I_{3}$ (т. е. $a_{1}>a_{2}>a_{3}$ ), тогда можно показать [9], что имеется три возможных типа бифуркационных диаграмм общего положения. С учетом возможного выбора знаков в соотношениях (18) получим, что бифуркационная диаграмма симметрична относительно смены знаков $c \rightarrow-c$, поэтому будем полагать $c \geqslant 0$.

1. $\frac{c}{\sqrt{\mu \operatorname{det} \mathbf{I}}}<\sqrt{a_{1}-a_{3}}-\sqrt{a_{2}-a_{3}}$.

В этом случае бифуркационное множество $\Sigma_{1}$ состоит из трех кривых (см. рис. 7):

$\gamma_{1}(\lambda):\left(f_{+}(\lambda), h_{+}(\lambda)\right), \lambda \in\left(I_{3}, \lambda_{11}\right)$, где $\lambda_{11} \in\left(I_{3},+\infty\right)$ является корнем уравнений $h_{+}\left(\lambda_{11}\right)=h_{1}^{(0)}, f_{+}\left(\lambda_{11}\right)=f_{1}^{(0)}$;

$\gamma_{2}(\lambda):\left(f_{+}(\lambda), h_{+}(\lambda)\right), \lambda \in\left(I_{1}, \lambda_{21}\right)$, где $\lambda_{21} \in\left(I_{1}, I_{2}\right)$ - корень уравнений $h_{+}\left(\lambda_{21}\right)=h_{1}^{(0)}, f_{+}\left(\lambda_{21}\right)=f_{1}^{(0)}$

$\gamma_{3}(\lambda):\left(f_{-}(\lambda), h_{-}(\lambda)\right), \lambda \in\left(\lambda_{22}, I_{2}\right)$, где $\lambda_{22} \in\left(I_{1}, I_{2}\right)$ - корень уравнений $h_{-}\left(\lambda_{22}\right)=h_{2}^{(0)}, f_{-}\left(\lambda_{22}\right)=f_{2}^{(0)}$.

При этом кривые $\gamma_{1}, \gamma_{2}$ выходят из точки $P_{1} \in \Sigma_{0}$, а кривая $\gamma_{3}$ выходит из точки $P_{2} \in \Sigma_{0}$ и имеет точку самопересечения $P_{3} \in \Sigma_{0}$. 


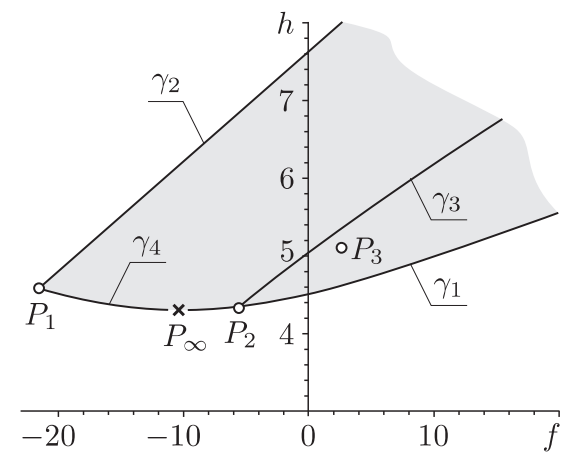

Рис. 8. Бифуркационная диаграмма при условии $\sqrt{a_{1}-a_{3}}-\sqrt{a_{2}-a_{3}}<$ $\frac{c}{\sqrt{\mu \operatorname{det} \mathbf{I}}}<\sqrt{a_{1}-a_{3}}+\sqrt{a_{2}-a_{3}}$. Параметры: $I_{1}=3, I_{2}=5, I_{3}=7$, $\mu=1, c=4.3$. Точка $P_{\infty}$ не является критической, в ней сходятся ветви $\left.\gamma_{1}\right|_{\lambda \rightarrow \infty}$ и $\left.\gamma_{4}\right|_{\lambda \rightarrow \infty}\left(P_{\infty} \notin \Sigma_{0}\right)$.

Здесь $P_{i}=\left(h_{i}^{(0)}, f_{i}^{(0)}\right)$ определены соотношениями (20).

2. $\sqrt{a_{1}-a_{3}}-\sqrt{a_{2}-a_{3}}<\frac{c}{\sqrt{\mu \operatorname{det} \mathbf{I}}}<\sqrt{a_{1}-a_{3}}+\sqrt{a_{2}-a_{3}}$.

В этом случае критическое множество $\Sigma_{1}$ состоит из четырех кривых (см. рис. 8):

$\gamma_{1}(\lambda):\left(f_{+}(\lambda), h_{+}(\lambda)\right), \lambda \in\left(I_{3},+\infty\right)$

$\gamma_{2}(\lambda):\left(f_{+}(\lambda), h_{+}(\lambda)\right), \lambda \in\left(I_{1}, \lambda_{21}\right)$, где $\lambda_{21} \in\left(I_{1}, I_{2}\right)$ - корень уравнений $h_{+}\left(\lambda_{21}\right)=h_{1}^{(0)}, f_{+}\left(\lambda_{21}\right)=f_{1}^{(0)}$

$\gamma_{3}(\lambda):\left(f_{-}(\lambda), h_{-}(\lambda)\right), \lambda \in\left(\lambda_{22}, I_{2}\right)$, где $\lambda_{22} \in\left(I_{1}, I_{2}\right)$ - корень уравнений $h_{-}\left(\lambda_{22}\right)=h_{2}^{(0)}, f_{-}\left(\lambda_{22}\right)=f_{2}^{(0)}$

$\gamma_{4}(\lambda):\left(f_{-}(\lambda), h_{-}(\lambda)\right), \lambda \in\left(\lambda_{32}, \lambda_{31}\right)$, где $\lambda_{32}, \lambda_{31} \in(-\infty, 0)$ - корни уравнений $h_{-}\left(\lambda_{32}\right)=h_{2}^{(0)}, f_{-}\left(\lambda_{32}\right)=f_{2}^{(0)}, h_{-}\left(\lambda_{31}\right)=h_{1}^{(0)}, f_{-}\left(\lambda_{31}\right)=f_{1}^{(0)}$.

Кривая $\gamma_{2}$ выходит из точки $P_{1} \in \Sigma_{0}$, кривые $\gamma_{1}, \gamma_{3}$ - из точки $P_{2} \in \Sigma_{0}$, кривая $\gamma_{4}$ заключена между точками $P_{1}, P_{2}$, критическая точка $P_{3} \in \Sigma_{0}$ находится внутри области возможных интегралов вне всех кривых $\gamma_{i}$.

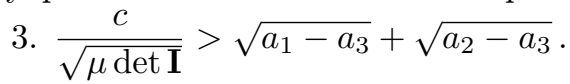

В этом случае $\Sigma_{1}$ состоит из четырех кривых (см. рис. 9):

$\gamma_{1}(\lambda):\left(f_{+}(\lambda), h_{+}(\lambda)\right), \lambda \in\left(I_{3}, \lambda_{13}\right)$, где $\lambda_{13} \in\left(I_{3},+\infty\right)$ - корень уравнений $h_{+}\left(\lambda_{13}\right)=h_{3}^{(0)}, f_{+}\left(\lambda_{13}\right)=f_{3}^{(0)}$

$\gamma_{2}(\lambda):\left(h_{+}(\lambda), f_{+}(\lambda)\right), \lambda \in\left(I_{1}, \lambda_{21}\right)$, где $\lambda_{21} \in\left(I_{1}, I_{2}\right)$ - корень уравнений $h_{+}\left(\lambda_{21}\right)=h_{1}^{(0)}, f_{+}\left(\lambda_{21}\right)=f_{1}^{(0)}$

$\gamma_{3}(\lambda):\left(h_{-}(\lambda), f_{-}(\lambda)\right), \lambda \in\left(\lambda_{22}, I_{2}\right)$, где $\lambda_{22} \in\left(I_{1}, I_{2}\right)$ - корень уравнений $h_{-}\left(\lambda_{22}\right)=h_{2}^{(0)}, f_{-}\left(\lambda_{22}\right)=f_{2}^{(0)}$

$\gamma_{4}(\lambda):\left(h_{-}(\lambda), f_{-}(\lambda)\right), \lambda \in\left(\lambda_{32}, \lambda_{31}\right)$, где $\lambda_{31}, \lambda_{32} \in(-\infty, 0)$ - корни уравнений $h_{-}\left(\lambda_{31}\right)=h_{1}^{(0)}, f_{-}\left(\lambda_{31}\right)=f_{1}^{(0)}, h_{-}\left(\lambda_{32}\right)=h_{2}^{(0)}, f_{-}\left(\lambda_{32}\right)=f_{2}^{(0)}$.

Кривая $\gamma_{1}$ выходит из точки $P_{3} \in \Sigma_{0}$, кривая $\gamma_{2}$ - из точки $P_{1} \in \Sigma_{0}$, кривая $\gamma_{3}$ - из точки $P_{2} \in \Sigma_{0}$, кривая $\gamma_{4}$ заключена между точками $P_{1}, P_{2}$. 


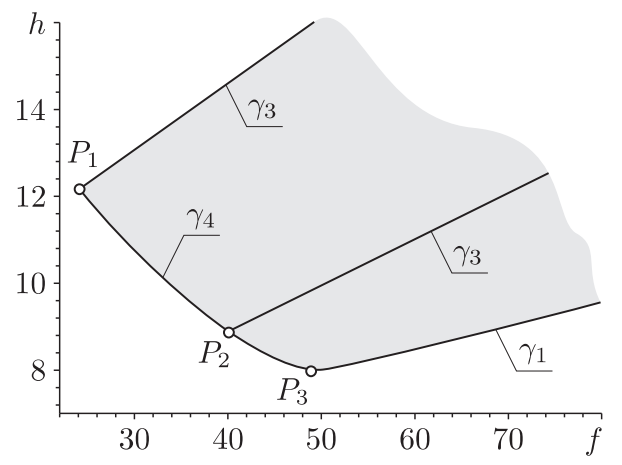

Рис. 9. Бифуркационная диаграмма при условии $\frac{c}{\sqrt{\mu \operatorname{det} \mathbf{I}}}>\sqrt{a_{1}-a_{3}}+$ $\sqrt{a_{2}-a_{3}}$. Параметры: $I_{1}=3, I_{2}=5, I_{3}=7, \mu=1, c=8$.

\section{3. Устойчивость периодических траекторий}

В предыдущем разделе мы выделили несколько естественных типов замкнутых траекторий, характерных для интегрируемых гамильтоновых систем с двумя степенями свободы, а именно:

1) некритические траектории двух видов:

- лежащие на торах Лиувилля;

- лежащие на особых интегральных многообразиях;

2) критические траектории:

- невырожденные (в смысле теории особенностей):

- эллиптические;

- гиперболические;

- вырожденные.

Согласно общей схеме бифуркационного анализа, которой мы следуем в настоящей работе, при решении вопроса об устойчивости замкнутой траектории требуется прежде всего определить ее тип. Если это уже сделано, то за исключением случая вырожденных траекторий ответ сразу дается при помощи двух почти очевидных утверждений, обсуждаемых ниже (теоремы 4, 5). Затем мы сформулируем несколько более общих утверждений (теоремы 6,7 и 8), с помощью которых можно определить устойчивость и в тех случаях, когда рассматриваемая замкнутая траектория вырождена или проверка невырожденности затруднена.

3.1. Случай некритических периодических траекторий. Говоря об анализе устойчивости некритических периодических траекторий, отметим прежде всего, что их мультипликаторы равны единице. Следующее простое утверждение относится к некритическим траекториям (оно никак не зависит от их типа, т. е. от того, лежат ли они на торе или на особом интегральном многообразии).

Обратим также внимание на то, что при исследовании устойчивости динамической системы стандартными методами ситуация единичных мультипликаторов является наиболее сложной. В предлагаемой нами схеме единичные муль- 
типликаторы отнюдь не приводят ни к каким дополнительным трудностям. В качестве примера подобной ситуации можно привести случай Горячева-Чаплыгина (см. п. 4.1). В этом собственно и есть основное преимущество подхода, основанного на анализе структуры особенностей слоения на торы.

Так, справедлива следующая теорема.

ТЕОРема 4. Если рассматриваемая гамильтонова система нерезонансна, то некритические замкнутые траектории всегда неустойчивы.

ДокАЗАТЕльство. Если некритическая периодическая траектория $\gamma$ лежит на (резонансном) торе Лиувилля, то ее неустойчивость совершенно очевидна. Действительно, вблизи от данного тора обязательно находится нерезонансный, на котором траектории всюду плотны. Ясно, что такие траектории не могут целиком содержаться в малой окрестности $\gamma$.

Если траектория $\gamma$ лежит на особой интегральной поверхности (но сама критической не является!), то доказательство на самом деле ничем не отличается от предыдущего рассуждения. Поскольку нерезонансные торы всюду плотны, то через любую ее окрестность такой тор проходит. Более того, этот тор не может остаться ни в какой малой окрестности этой траектории, поскольку он "примыкает" не только к самой траектории, но и к двумерному интегральному многообразию, на котором лежит эта траектория. А это многообразие "большое", т. е. не содержится в малой окрестности траектории. Поскольку любой близкий тор покидает окрестность траектории, в силу нерезонансности покидать ее будут и все траектории, лежащие на близком нерезонансном торе. Теорема 4 доказана.

Если для некритических замкнутых траекторий проводить стандартный анализ устойчивости при помощи рассмотрения дифференциала отображения Пуанкаре, то мы увидим, что его собственные значения равны единице. Обычно при этом будет возникать жорданова клетка, что будет являться свидетельством неустойчивости. Но даже если вместо клетки мы имеем тождественный оператор, согласно топологическому анализу, проведенному выше, гарантированно получим неустойчивость.

Нерезонансность системы при этом, разумеется, важна. Грубо говоря, альтернатива здесь такова: либо вообще все траектории в окрестности некритической замкнутой траектории являются замкнутыми (резонансный случай), либо мы имеем неустойчивость (нерезонансный случай). В терминах отображения Пуанкаре это означает следующее: в окрестности некритической замкнутой траектории отображение Пуанкаре либо тождественно, либо неустойчиво.

Решение Колосова. Как уже отмечалось, явного разделения переменных для случая Клебша не найдено. Тем не менее при попытках интегрирования случая Клебша были найдены различные частные решения, допускающие такое разделение. Любопытное и достаточно сложное семейство частных решений было получено Г. Колосовым [72]. На этом пути он нашел систему дробно-рациональных интегралов, которые задают выделенный тор в фазовом пространстве. Колосов в своем подходе ограничивается аналитическим подходом, получая решение в форме квадратур. Вследствие своей сложной формы 
эти квадратуры не дают полезной информации о качественных свойствах решения. Ниже мы приводим более явный анализ его устойчивости.

Для этого семейства предполагается, что главные моменты инерции связаны соотношением

$$
I_{3}=I_{1}+I_{2}
$$

т. е. тело представляет собой плоскую пластинку.

В этом случае, если величины интеграла Клебша и гамильтониан (6) связаны линейным соотношением

$$
a_{3} f-2 h+\frac{a_{3}^{2}+a_{1} a_{2}}{\operatorname{det} \mathbf{A}}=0,
$$

то система допускает дополнительный дробно-рациональный интеграл

$$
G=\frac{a_{1} M_{1} \gamma_{1}+a_{2} M_{2} \gamma_{2}}{I_{1} \gamma_{2}^{2}-a_{1} M_{1}^{2}}
$$

здесь для простоты полагается $\mu=1,(\gamma, \gamma)=1$. При этом решение дается в квадратурах, так что

$$
\dot{\gamma}_{3}^{2}=\left(1-\gamma_{3}^{2}\right)^{2}-\frac{g^{2}}{I_{1}-I_{2}}\left(\frac{\left(I_{1}-I_{2}\right)^{2}}{I_{1} I_{3}} \frac{\left(g_{1}-\gamma_{3}\right)^{2}}{g^{2}}-2 h I_{1}+I_{1} I_{3}\right)^{2}
$$

здесь $g$ - значение дробно-рационального интеграла (22), а $g_{1}$ - величина еще одного (зависимого) интеграла, который имеет вид

$$
G_{1}=\gamma_{3}-\frac{I_{3}-I_{2}}{I_{2}-I_{1}} G M_{3}
$$

Ясно, что при таких громоздких формулах стандартный подход к исследованию устойчивости (основанный на вычислении матрицы монодромии) приводит к довольно длинным вычислениям. С другой стороны, провести прямую (21) на соответствующих бифуркационных диаграммах не представляет труда (см. рис. 10). Как видно на рисунке, прямая (21) всегда проходит через особую точку $P_{3}$ (это несложно понять аналитически).

Таким образом, мы заключаем, что в общем случае решение Колосова (23) описывает периодические решения на семействе резонансных торов и, согласно сказанному выше (см. теорему 4), эти движения неустойчивы.

3.2. Случай невырожденных периодических траекторий. При анализе устойчивости невырожденных (в смысле теории особенностей) траекторий никаких проблем не возникает. Если рассматриваемая система нерезонансна, то имеет место следующее утверждение.

Теорема 5. Эллиптические невырожденные траектории устойчивы, гиперболические невырожденные траектории неустойчивы.

Утверждение, разумеется, очевидно, но в качестве формального доказательства достаточно заметить, что оно является непосредственным следствием теорем 2,3 . 


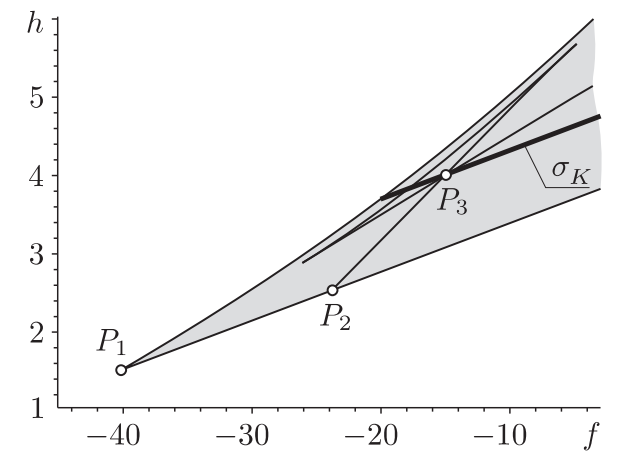

a)

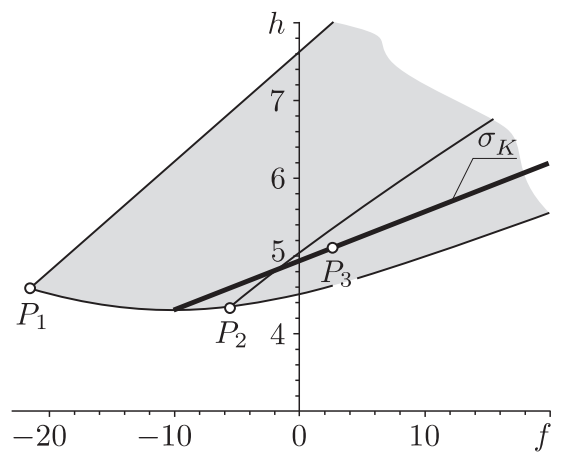

b)

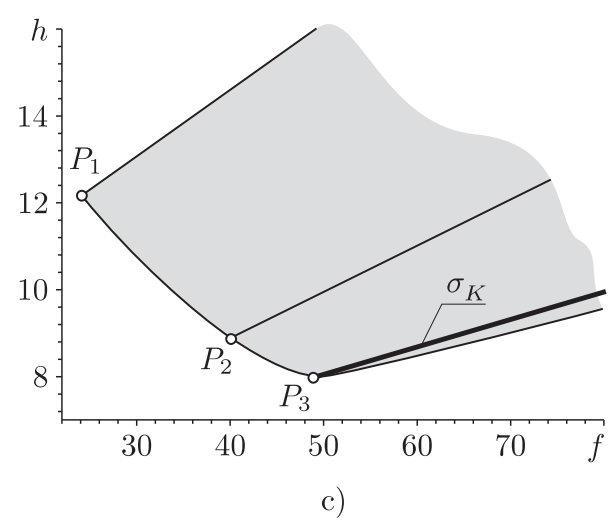

Рис. 10. Бифуркационные диаграммы случая Клебша при условии $I_{3}=I_{1}+I_{2}\left(I_{1}=3, I_{2}=5, I_{3}=8, \mu=1\right)$ и прямая $\sigma_{K}$, соответствующая периодическим решениям Колосова. а) $(\mu \operatorname{det} \mathbf{I})^{-1 / 2} c<\sqrt{a_{1}-a_{3}}-$ $\sqrt{a_{2}-a_{3}}$, b) $\sqrt{a_{1}-a_{3}}-\sqrt{a_{2}-a_{3}}<(\mu \operatorname{det} \mathbf{I})^{-1 / 2} c<\sqrt{a_{1}-a_{3}}+\sqrt{a_{2}-a_{3}}$, c) $(\mu \operatorname{det} \mathbf{I})^{-1 / 2} c>\sqrt{a_{1}-a_{3}}+\sqrt{a_{2}-a_{3}}$.

Говоря об устойчивости, мы имеем здесь в виду устойчивость на симплектическом многообразии. В частности, если рассматриваемое многообразие не симплектическое, а пуассоново, то утверждение теоремы, строго говоря, относится лишь к симплектическому листу, на котором лежит рассматриваемая траектория. Переход на соседние симплектические листы может рассматриваться как малое возмущение интегрируемой системы, и вопросы устойчивости в такой ситуации придется решать уже при помощи KAM-теории. Однако в данной ситуации подобных проблем не возникает, если мы предполагаем интегрируемость в целом на пуассоновом многообразии: утверждение теоремы будет справедливым не только на отдельном симплектическом листе, но и в целом на пуассоновом многообразии. При этом нам даже не требуется предполагать нерезонансность на выделенном симплектическом листе, достаточно нерезонансности на пуассоновом многообразии в целом. 
3.3. Общий критерий устойчивости. I. Если заранее не ясно, к какому типу относится замкнутая траектория, то можно использовать следующий совершенно общий и легко формулируемый критерий устойчивости любой замкнутой траектории интегрируемой гамильтоновой системы.

Теорема 6. Замкнутая траектория интегрируемой гамильтоновой системы устойчива тогда и только тогда, когда связная компонента интегрального многообразия, содержащая эту траекторию, совпадает с ней самой.

ДокАзАТЕльство. Доказательство в одну сторону, когда траектория $\gamma$ совпадает с интегральным подмногообразием, т. е. имеет вид $\gamma=\left\{H=h_{0}, F=f_{0}\right\}$, является простым. Достаточно рассмотреть функцию $\left(H-h_{0}\right)^{2}+\left(F-f_{0}\right)^{2}$. Это интеграл системы, имеющий на данной траектории минимум. Тем самым эта функция вполне может служить в качестве функции Ляпунова, гарантирующей орбитальную устойчивость.

Доказательство обратного утверждения сводится к анализу особых (критических) точек функции двух переменных и может быть проведено следующим образом.

Рассмотрим двумерную изоэнергетическую трансверсаль $\mathscr{N}=\mathscr{N}_{h_{0}}$ к траектории $\gamma$ и ограничим на нее функцию $F$. Согласно нашим предположениям эта функция аналитическая, не константа и имеет особенность в точке $P_{0}=\gamma \cap \mathscr{N}$, которая, очевидно, будет неподвижной точкой отображения Пуанкаре $\sigma: \mathscr{N} \rightarrow \mathscr{N}$. Мы хотим на самом деле показать неустойчивость этого отображения. (Если точка $P_{0}$ не является особой для $\left.F\right|_{\mathscr{N}}$, то доказательство упрощается, но проводится по той же схеме.)

Мы воспользуемся следующим фактом из теории аналитических функций. Множество критических точек аналитической функции является локально связным. Это означает, что в $\mathscr{N}$ существует окрестность $U$ точки $P_{0}$ такая, что множество $S$ особых точек функции $\left.F\right|_{\mathscr{N}}$, попавших в $U$ (или, что то же самое, $S \cap U)$, связно.

Итак, предположим теперь, что траектория устойчива. Будем работать внутри указанной окрестности. Устойчивость означает, что мы можем найти меньшую окрестность $V$ такую, что итерированное отображение Пуанкаре, стартуя в $V$, никогда не покидает окрестности $U$. По нашему предположению, нерезонансные торы всюду плотны. Рассмотрим произвольный тор такого типа, проходящий через окрестность $V$, и связную компоненту его пересечения с окрестностью $U$. С точки зрения функции $\left.F\right|_{\mathscr{N}}$ это множество представляет собой неособую линию уровня, целиком лежащую в $U$ (в силу устойчивости). Поскольку линия уровня - неособая, она представляет собой гладко вложенную окружность. По теореме Жордана эта окружность делит $U$ на две области: внутреннюю и внешнюю. Мы утверждаем, что наша точка $P_{0}$ лежит внутри. Действительно, в противном случае внутри этой окружности обязательно нашлась бы еще одна критическая точка $P$ функции $\left.F\right|_{\mathscr{N}}$ и мы получили бы противоречие со связностью критического множества (точки $P_{0}$ и $P$ оказались бы разделенными описанной выше гладко вложенной окружностью). Таким образом, $P_{0}$ лежит внутри этой окружности. Рассматривая внутренность этой окружности как новую окрестность точки $P_{0}$, мы можем снова взять какой-то 
меньший тор, сидящий внутри этой окрестности, и повторить процедуру. Точка $P_{0}$ снова окажется внутри. При этом на каждом шаге мы имеем право уменьшать размер рассматриваемой окрестности таким образом, что в конце концов траектория $P$ окажется единственным пересечением этих вложенных друг в друга областей.

Возникает вопрос о том, как устроена связная компонента множества $\left.F\right|_{\mathscr{N}}=$ $f_{0}=F(P)$. Ясно, что это изолированная точка $P_{0}$. Отметим, что здесь мы пользуемся аналитичностью. Иначе мы бы снова сразу получили противоречие с локальной связностью множества $\left.F\right|_{\mathscr{N}}=f_{0}=F(P)$. Теорема 6 доказана.

В приведенном доказательстве мы предполагали, что нерезонансные торы всюду плотны не только в четырехмерном фазовом пространстве, но и на рассматриваемом трехмерном уровне энергии $H=h_{0}$. Строго говоря, это дополнительное предположение. Однако никакой проблемы с доказательством в общем случае не возникает. Даже если все "изоэнергетические" торы резонансны, мы можем сместиться в сторону, слегка изменив значение энергии, и там уже будет нерезонансный тор.

Сделаем несколько замечаний по поводу приведенной выше теоремы.

1. В этой теореме мы исключаем из рассмотрения вырожденную ситуацию, когда трехмерный изоэнергетический уровень $\left\{H=h_{0}\right\}$ целиком состоит из критических точек интегрального отображения. Другими словами, мы предполагаем, что интеграл $F$ на этом уровне нетривиален, т. е. не является тождественной константой.

2. В формулировке теоремы совершенно неважно, является ли рассматриваемая траектория критической или некритической, вырожденной или невырожденной. В этом смысле утверждение носит универсальный характер.

3. Предположение об аналитичности в доказательстве было использовано весьма существенно.

4. Утверждение теоремы остается справедливым и в том случае, когда мы имеем дело не с четырехмерным симплектическим многообразием, а с пуассоновым многообразием, расслоенным на четырехмерные симплектические листы. Неустойчивость на "большем" пуассоновом многообразии, разумеется, следует из неустойчивости на симплектическом листе. С устойчивостью также никаких проблем не возникает. Как мы видели, на симплектическом листе для устойчивой траектории в качестве функции Ляпунова всегда можно взять функцию $\left(H-h_{0}\right)^{2}+\left(F-f_{0}\right)^{2}$. Совершенно аналогичным образом для доказательства устойчивости этой траектории в объемлющем пуассоновом многообразии функцию Ляпунова можно получить, добавив к $\left(H-h_{0}\right)^{2}+\left(F-f_{0}\right)^{2}$ квадраты функций Казимира.

5. Этот критерий устойчивости естественным образом обобщается на системы с бо́льшим числом степеней свободы.

\section{4. Устойчивость семейств периодических траекторий и бифурка-} ционная диаграмма. Как мы видели выше (следствие 1), невырожденные критические периодические траектории объединяются в однопараметрические семейства, которые интегральным отображением $\Phi$ переводятся в бифуркационные кривые множества $\Sigma_{1}$. Обычно верно и обратное, что позволяет эффективно использовать бифуркационную диаграмму интегрального отображения 
для анализа устойчивости. А именно, практически во всех известных примеpax, для которых проведен топологический анализ (см., например, [14]), справедливо следующее:

1) гладкой ветви бифуркационной диаграммы соответствует однопараметрическое семейство (или несколько не связанных между собой семейств) невырожденных критических траекторий;

2) тип траектории семейства (эллиптический/гиперболический) не может изменяться в неособых точках ветви (т. е. смена типа происходит в точках пересечения ветвей, излома, возврата и т. п.).

Таким образом, грубо говоря, для анализа устойчивости критических траекторий необходимо определить тип траектории для каждой кривой из бифуркационного множества $\Sigma$. При этом достаточно определить тип траектории (эллиптический/гиперболический) в какой-нибудь одной из точек $(h, f) \in \Sigma$ гладкой ветви бифуркационной диаграммы.

Поясним, что под семейством критических периодических траекторий мы понимаем однопараметрическое семейство $\gamma_{\lambda}$ периодических траекторий, гладко зависящих от параметра $\lambda$, пробегающего некоторый интервал $(a, b)$. Каждая траектория $\gamma_{\lambda}$ предполагается критической, т. е. $\gamma_{\lambda} \subset S_{1}$, и, кроме того, мы, разумеется предполагаем, что $\gamma_{\lambda} \neq \gamma_{\mu}$ при $\lambda \neq \mu$. Напомним, что в интегрируемой системе имеются также однопараметрические семейства некритических замкнутых траекторий, лежащие на резонансных торах Лиувилля, но мы сейчас обсуждаем совершенно иную ситуацию.

Как мы видели выше при построении бифуркационной диаграммы случая Клебша, однопараметрическому семейству периодических орбит $\gamma_{\lambda} \subset S_{1}$, аналитически зависящих от параметра $\lambda$, на бифуркационной диаграмме может соответствовать кривая с самопересечениями и точками возврата (каспами). Как известно [29], точке возврата соответствуют вырожденные траектории, в которых обычно происходит смена типа с эллиптического на гиперболический и наоборот. Поэтому в дальнейшем под отделъной ветвъю бифуркационной диаграммы мы будем понимать гладкий участок кривой из множества $\Sigma_{1}$, не содержащий ни точек пересечения с другими ветвями, ни точек возврата, ни точек излома и т. п.

Теоретически, однако, нельзя исключать ситуацию, когда все семейство, кроме отдельных траекторий, состоит из невырожденных критических траекторий одного и того же типа и при этом образ семейства на бифуркационной диаграмме выглядит как гладкая ветвь (без точек пересечения и возврата). В этом случае диагностика присутствия вырождений в семействе может оказаться затруднительной.

Однако если речь идет лишь об анализе устойчивости, то наша задача значительно облегчается. Дело в том, что встречающиеся в гладком семействе, отображающемся на одну и ту же ветвь, отдельные вырожденные траектории обязаны иметь тот же тип устойчивости, что и остальные (невырожденные) траектории. Другими словами, устойчивость не может возникнуть или потеряться для отдельной изолированной траектории. А именно, имеет место следующее утверждение, которое может служить хорошей основой для анализа устойчивости в интегрируемых системах. 
ТЕОРема 7. Рассмотрим однопараметрическое семейство замкнутых критических траекторий. Предположим, что это семейство изолировано в том смысле, что никаких других критических точек интегрального отображения в окрестности этого семейства нет. Предположим далее, что это семейство отображается на некоторую отдельную ветвъ бифуркационной диаграммы $\Sigma$, которая может быть задана как график некоторой гладкой функции $F=f(H)$.

Пусть хотя бы одна траектория из семейства является невырожденной. Тогда:

1) почти все траектории семейства невырождены и имеют один и тот же тип (либо эллиптический, либо гиперболический);

2) если одна из траекторий семейства имеет эллиптический тип, то все траектории семейства (как вырожденные, так и невырожденные) устойчивы;

3) напротив, если хотя бы одна из траекторий имеет гиперболический тип, то все траектории семейства (как вырожденные, так и невырожденные) неустойчивы.

Это утверждение, в частности, говорит, что гиперболические траектории не могут стать эллиптическими внутри семейства, если это семейство отображается в гладкую ветвь бифуркационной диаграммы и к этому семейству не примыкают никакие другие семейства (в формулировке теоремы это условие называется изолированностью семейства).

Доказательство теоремы будет дано ниже в приложении А.1.

\section{5. Общий критерий устойчивости. II. Бифуркационный комп-} лекс. Здесь мы хотим описать еще один чисто топологический способ определения устойчивости, основанный по существу на анализе бифуркационной диаграммы.

Прежде всего отметим случай, когда для определения устойчивости не требуется определять тип траектории. А именно, верно следующее утверждение.

ПрЕДЛОЖЕНИЕ 2. Если некоторое семейство периодических траекторий, удовлетворяет условиям теоремы 7 и соответствует ветви бифуркационной диаграммы, лежащей на краю ОВД, то все траектории из этого семейства устойчивы.

Доказательство этого утверждения несложное. Мы не будем приводить его отдельно, а сошлемся на общую теорему 8, к обсуждению которой мы сейчас и приступаем.

Оказывается, что если соответствующим образом обобщить понятие бифуркационной диаграммы, данное выше (см. п. 2.1), то будет справедливым и обратное утверждение о том, что "устойчивая ветвь" лежит только на границе ОВД.

Чтобы описать соответствующее обобщение (мы будем называть его бифуркационным комплексом), заметим, что на бифуркационной диаграмме имеются регулярные точки $(h, f) \in \Phi(\mathscr{M}) \backslash \Sigma$, которым соответствует более чем одна связная компонента (тор) общего интегрального многообразия $\mathscr{M}_{h, f}$. Это удобно представлять себе так, что над этой точкой имеется несколько различных 
листов области возможных значений интегралов (по аналогии с поверхностью Римана многозначной аналитической функции).

Ясно, что различные листы могут склеиваться только вдоль ветвей бифуркационного множества $\Sigma$. Говоря неформально, эту совокупность склеенных листов и кривых мы назовем бифуркационным комплексом.

С математически более строгой точки зрения бифуркащионный комплекс представляет собой топологическое пространство, точками которого будут по определению связные компоненты интегральных многообразий $\mathscr{M}_{h, f}$ с естественной фактортопологией. Несколько иначе эту же мысль можно выразить, сказав, что бифуркационный комплекс получается из многообразия $\mathscr{M}^{4}$ при сжатии в точку каждой связной компоненты интегральных многообразий $\mathscr{M}_{h, f}$. Другими словами, это пространство слоев, или база сингулярного лагранжева слоения, задаваемого первыми интегралами гамильтоновой системы.

С топологической точки зрения бифуркационный комплекс для систем с "хорошими" интегралами представляет собой клеточный комплекс, который можно понимать как топологический инвариант рассматриваемой интегрируемой системы.

Естественным образом определяется соответствующее обобщение $\widetilde{\Phi}$ интегрального отображения (7): каждой точке $\boldsymbol{x} \in \mathscr{M}$ ставится в соответствие та точка бифуркационного комплекса $\mathscr{K}$, которая задает связную компоненту интегрального многообразия, на котором данная точка лежит. Сам бифуркационный комплекс в свою очередь естественным образом проецируется на пространство первых интегралов $\mathbb{R}^{2}(h, f)$ :

$$
\pi: \mathscr{K} \rightarrow \mathbb{R}^{2}(h, f)
$$

так, что при этом $\Phi=\pi \circ \widetilde{\Phi}$.

Теперь можно сформулировать некий общий принцип, позволяющий очень просто определять устойчивость замкнутых траекторий интегрируемых систем: периодическая траектория $\gamma$ устойчива тогда и только тогда, когда ее образ при отображении $\widetilde{\Phi}$ лежит на гранище бифуркащионного комплекса.

Чтобы этот принцип сделать теоремой, необходимы некоторые дополнительные, но очень естественные предположения.

А именно, следует запретить существование двумерных кусков интегральных многообразий, целиком заполненных критическими траекториями. Такого типа дополнительное условие можно сформулировать, например, следующим образом.

ОПРЕДЕЛЕНИЕ. Мы скажем, что отображение момента $\Phi$ удовлетворяет $y c-$ ловию конечности орбит, если каждое интегральное многообразие $\mathscr{M}_{h, f}$ является объединением конечного числа орбит пуассонова действия группы $\mathbb{R}^{2}$, порожденного коммутирующими функциями $H$ и $F$.

Геометрически это означает, что $\mathscr{M}_{h, f}$ представляет собой многообразие с особенностями, которое естественным образом распадается на конечное число непересекающихся кусков размерности 0,1 или 2, каждый из которых уже является настоящим гладким подмногообразием (не обязательно замкнутым). Для аналитических функций это условие всегда выполнено, но нам нужно еще 
одно дополнение: мы хотим, чтобы ранг интегрального отображения $\Phi$ на куске размерности $k$ был в точности равен $k$, где $k=0,1,2$ (так называемая стратификация по рангу). Последнее эквивалентно тому, что каждый кусок является орбитой соответствующего пуассонова действия.

Можно, впрочем, переформулировать нужное нам условие еще и так: $\mathscr{M}_{h, f}$ содержит лишь конечное число критических траекторий.

Кроме этого дополнительного условия нам следует также разъяснить, что мы будем понимать под граничной точкой бифуркационного комплекса.

Если бы речь шла не о бифуркационном комплексе, а о бифуркационной диаграмме, то определение граничной точки было бы очевидным: точка $\left(h_{0}, f_{0}\right) \in$ $\mathbb{R}^{2}(h, f)$ называется граничной, если в произвольной ее окрестности есть как точки, принадлежащие ОВД, так и точки, не принадлежащие ОВД. В случае бифуркационного комплекса ситуация практически та же самая. Нужно лишь не забывать, что комплекс может иметь несколько различных листов над плоскостью первых интегралов. Каждой точке $P \in \mathscr{K}$ мы по-прежнему можем сопоставить значения $h$ и $f$, но может существовать несколько точек, отвечающих одним и тем же значениям интегралов. С топологической точки зрения это просто означает, что нам следует рассмотреть естественную проекцию $\pi: \mathscr{K} \rightarrow \mathbb{R}^{2}(h, f)$. Определение граничной точки комплекса будет точно таким же, как выше, но вместо окрестности точки $P$ придется рассмотреть ее проекцию.

ОПРЕДЕЛЕНИЕ. Пусть $\mathscr{K}-$ бифуркационный комплекс, $P \in \mathscr{K}$ и $U(P)-$ достаточно малая окрестность $P$ в $\mathscr{K}$. Мы скажем, что точка $P$ лежит на границе бифуркационного комплекса (или является граничной), если ее проекция $\left(h_{0}, f_{0}\right)$ на плоскость первых интегралов является граничной точкой по отношению к проекции окрестности $U(P)$.

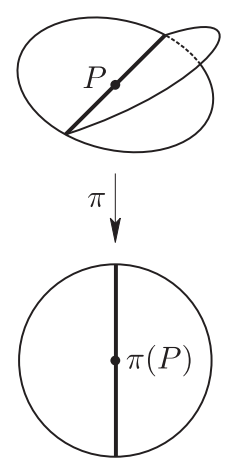

внутренняя

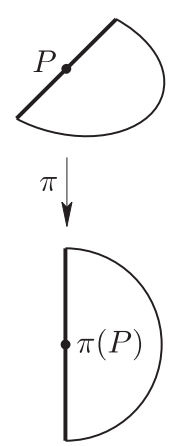

граничная

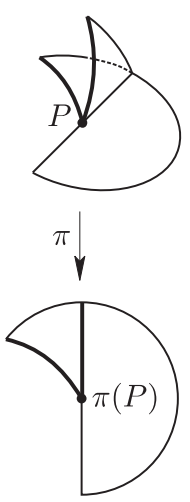

граничная

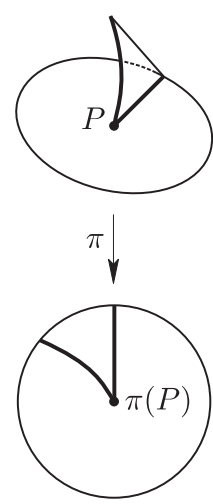

внутренняя

Рис. 11. Несколько примеров граничных и внутренних точек на бифуркационном комплексе

На рис. 11 показано несколько характерных примеров. Важно, что эти примеры включают и “точки ветвления", в которых сходятся несколько листов 
бифуркационного комплекса. При соблюдении этих необходимых предосторожностей мы имеем следующее утверждение.

ТЕОРема 8. Пусть отображение момента интегрируемой системы удовлетворяет условию конечности. Периодическая траектория $\gamma$ устойчива тогда и только тогда, когда ее образ при отображении $\widetilde{\Phi}$ лежит на гранище бифуркационного комплекса.

ДокАЗАтЕльство. По существу это утверждение является просто наглядной и удобной интерпретацией общего критерия, даваемого теоремой 6 . Действительно, возможны два случая, которые мы рассмотрим последовательно. Без ограничения общности мы будем считать, что $h_{0}=0, f_{0}=0$ на рассматриваемой траектории.

Случай 1: замкнутая траектория $\gamma$ совпадает со связной компонентой совместного уровня интегралов $H$ и $F$. Мы знаем, что в этом случае траектория $\gamma$ устойчива. Покажем, что $\widetilde{\Phi}(\gamma)$ - граничная точка.

Рассмотрим изоэнергетическую поверхность $H=0$ в окрестности траектории $\gamma$. Эта поверхность локально является гладким подмногообразием, и дополнительный интеграл $F$ может быть рассмотрен как вещественно аналитическая функция на нем. Мы знаем, что уровень этой функции $F=f_{0}$ совпадает с самой траекторией. На самом деле нам дано, что траектория является связной компонентой уровня $\left.F\right|_{\{H=0\}}=0$, но в силу аналитичности мы можем использовать локальную связность, чтобы уменьшить окрестность так, что в ней кроме самой траектории никаких других решений уравнения $\left.F\right|_{\{H=0\}}=0$ не будет. Если рассмотреть теперь интеграл $F$ на этой окрестности с выброшенной траекторией, то функция $F$ будет в этой окрестности знакопостоянной. Будем считать ее для определенности положительной. Тогда пары вида $(h, f)=(0,-\delta), \delta>0$, не принадлежат проекции окрестности $U(\widetilde{\Phi}(\gamma))$, и, стало быть, точка $\widetilde{\Phi}(\gamma)$ является граничной. Заметим, что при доказательстве этого утверждения мы никак не использовали условие конечности. Таким образом, образ любой устойчивой траектории всегда попадает на границу бифуркационного комплекса.

Случай 2: замкнутая траектория $\gamma$ не совпадает со связной компонентой совместного уровня интегралов $H$ и $F$, т. е. эта компонента содержит данную траекторию как собственное подмножество. В этом случае мы имеем неустойчивость. Покажем, что $\widetilde{\Phi}(\gamma)$ является внутренней точкой бифуркационного комплекса (т. е. не является граничной). Из условия конечности сразу следует, что к траектории примыкает некоторый двумерный кусок. Этот двумерный кусок $L$, очевидно, отображается в ту же самую точку $\widetilde{\Phi}(\gamma)$. Сам по себе он является двумерной орбитой пуассонова действия и, в частности, интегралы $H$ и $F$ на нем независимы. Отступим немного в сторону от траектории $\gamma$ и рассмотрим некоторую точку $x$, принадлежащую этому куску $L$. Поскольку в окрестности точки $x$ интегралы независимы, то они локально принимают все возможные значения в некоторой окрестности нуля. Но это в точности и означает, что точка $P=\widetilde{\Phi}(L)=\widetilde{\Phi}(\gamma)$ является внутренней. Теорема 8 доказана.

Возникает естественный вопрос о том, как проверять условие конечности. И насколько условие конечности является типичным. 


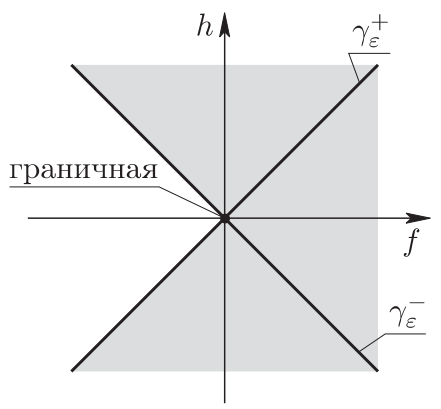

Рис. 12. Пример бифуркационной диаграммы системы с нарушением условий конечности

Прежде всего отметим, что условие конечности фактически проверяется само собой при построении бифуркационного множества. Обычно мы всегда имеем представление о том, как устроено критическое множество и каким образом оно отображается в плоскость интегралов. Условие конечности просто запрещает ситуации, при которых в одну и ту же точку множества $\Sigma$ отображается бесконечно много (т. е. целое однопараметрическое семейство) критических траекторий системы (необязательно замкнутых). В случаях Клебша и Горячева-Чаплыгина ясно, что этого не происходит: каждой точке из $\Sigma$ соответствует лишь конечное число критических траекторий.

Нарушение условия конечности. Приведем еще один пример, показывающий, каким образом условие конечности может нарушаться.

Рассмотрим следующую модель. Пусть $I_{1}, I_{2}, \phi_{1}, \phi_{2}$ - переменные действиеугол в окрестности обычного тора Лиувилля. Рассмотрим пару коммутирующих функций вида $H=I_{1}, F=I_{2}^{2}+I_{1} \cos \phi_{2}$ (если мы хотим иметь нерезонансную систему, то следует заменить $H$ на любую линейную комбинацию $H+\lambda F)$.

Множество критических точек этого интегрального отображения состоит из тора $I_{1}=I_{2}=0$ и двух семейств замкнутых траекторий $\gamma_{\varepsilon}^{+}=\left\{I_{2}=0, I_{1}=\varepsilon\right.$, $\left.\phi_{2}=0\right\}, \gamma_{\varepsilon}^{-}=\left\{I_{2}=0, I_{1}=\varepsilon, \phi_{2}=\pi\right\}$.

При отображении момента множество критических точек переходит в пару пересекающихся прямых $h= \pm f$. Это бифуркационное множество и область возможности движения показаны на рис. 12. Из этого рисунка сразу следует, что точка $h=0, f=0$ является граничной. Ее прообраз, однако, является тором $\left\{I_{1}=0, I_{2}=0\right\}$, целиком состоящим из критических траекторий. Все они неустойчивы.

Этот пример показывает, что вполне возможно появление отдельных “слабо предсказуемых" интегральных многообразий, для которых условие конечности нарушается. С другой стороны, этот пример также показывает, что при аккуратном бифуркационном анализе такие исключительные интегральные многообразия легко обнаруживаются. 


\section{4. Бифуркационные комплексы систем динамики твердого тела}

\section{1. Бифуркационный комплекс волчка Горячева-Чаплыгина.} Чтобы построить бифуркационный комплекс волчка Горячева-Чаплыгина, напомним, что в более темной области на рис. 6 каждой точке $(h, f)$ соответствует пара инвариантных торов, т. е. здесь имеется пара листов бифуркационного комплекса, а в точке касания $P_{1}$ происходит ветвление (см. рис. 13).

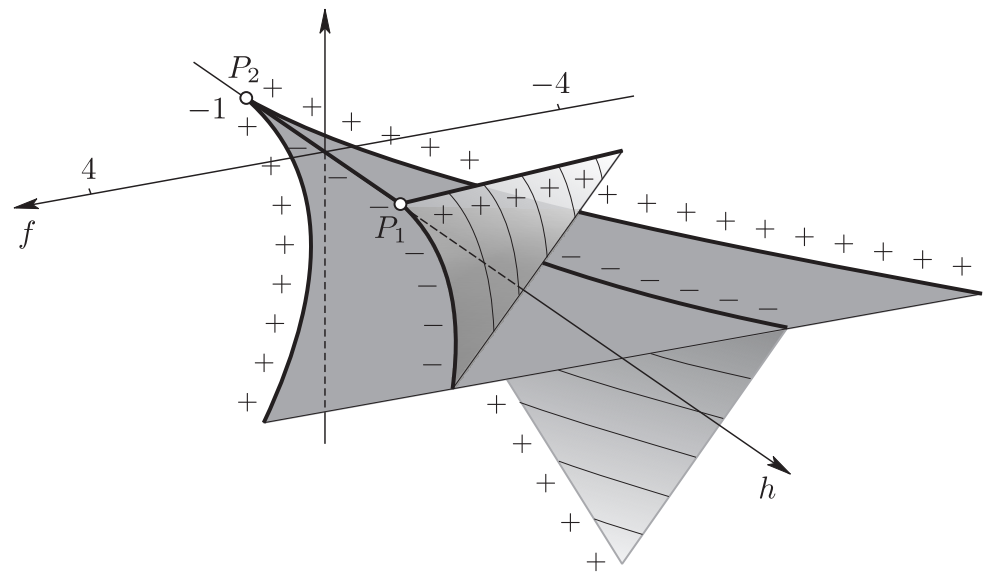

Рис. 13. Бифуркационный комплекс волчка Горячева-Чаплыгина. Знаком "+" помечены устойчивые критические периодические решения, а знаком "-" - неустойчивые.

Вертикальная ось на этом рисунке не имеет физического смысла и используется лишь для удобства визуализации различных листов. Устойчивость критических решений отмечена на рисунке знаками "+" (устойчивые) и “-” (неустойчивые).

Таким образом, мы видим, что для случая Горячева-Чаплыгина в фазовом пространстве системы имеется четыре семейства устойчивых периодических траекторий (других устойчивых орбит нет).

4.2. Бифуркационные комплексы случая Клебша. Возможные бифуркационные комплексы в случае Клебша, соответствующие бифуркационным диаграммам на рис. $7,8,9$, можно построить, пользуясь следующими наблюдениями (см. также рис. 11).

1. Различные листы бифуркационного комплекса могут склеиваться только по тем ветвям бифуркационной диаграммы, которые лежат внутри области возможных движений (в невырожденном случае им соответствуют гиперболические периодические решения), причем в этом случае на одном и том же (особом) слое $\mathscr{M}_{h, f}$ лежит несколько различных гиперболических решений.

2. Если точкам на граничных ветвях бифуркационной диаграммы соответствует несколько периодических траекторий в фазовом пространстве, то 
каждой такой траектории соответствует отдельный лист на бифуркационном комплексе.

3. Точки возврата (каспы на бифуркационной диаграмме), как правило, являются точками ветвления листов бифуркационного комплекса.

4. Количество листов и характер их склейки удобнее всего определить в окрестности точек множества $\Sigma_{0}$.

Для случая Клебша выше (см. п. 2.5) отмечалось, что каждой точке $P_{i} \in \Sigma_{0}$ соответствуют две неподвижные точки в фазовом пространстве и, аналогично, каждой точке на кривых $\gamma_{i} \in \Sigma_{1}$ соответствует пара периодических траекторий. Кроме того, можно показать, что точка $P_{2}$ и примыкающая к ней ветвь соответствуют решениям, лежащим на одном и том же интегральном многообразии $\mathscr{M}_{h, f}$ (это можно проверить численно, построив сечение Пуанкаре $\mathscr{N}_{h}$, либо анализируя перестройки областей возможного движения на сфере Пуассона, см. подробнее [9]-[12]).

Пользуясь этими наблюдениями, несложно построить бифуркационные комплексы при условии $(\mu \operatorname{det} \mathbf{I})^{-1 / 2} c>\sqrt{a_{1}-a_{3}}-\sqrt{a_{2}-a_{3}}$ (см. рис. 15,16$)$, т. е. для бифуркационных диаграмм на рис. 8, 9. Для случая малых $c$ (рис. 7) соответствующий бифуркационный комплекс не может быть вложен в трехмерное пространство, мы представим его в виде склейки отдельных листов на рис. 14.

Таким образом, пользуясь бифуркационными комплексами на рис. 14-16 несложно описать все устойчивые и неустойчивые критические периодические траектории и неподвижные точки для случая Клебша. Отметим лишь, что нужно быть аккуратным при “чтении" этих бифуркационных комплексов, так, например, нужно помнить, что кривая $\gamma_{3}$, хотя и не расслаивается на этих рисунках, тем не менее соответствует паре семейств неустойчивых траекторий, лежащих на одном и том же особом слое.

Окончательно получим:

в случае Клебша при всяком значении постоянной площадей с имеется шесть однопараметрических семейств устойчивых периодических орбит (которые на рис. 14-16 отмечены знаком “+"), других устойчивых периодических траекторий нет.

\section{5. Система Гаффэ}

Рассмотрим еще одну интегрируемую систему, которая может быть записана в гамильтоновой форме со скобкой Ли-Пуассона, соответствующей алгебpe $e(3)$. Эта система описывает эволюцию относительных размеров расширяющегося газового эллипсоида, заполненного одноатомным идеальным газом. Гамильтониан в этом случае имеет вид

$$
H=\frac{1}{2} \mathbf{M}^{2}+\frac{3}{2} \frac{a}{\left(\gamma_{1} \gamma_{2} \gamma_{3}\right)^{2 / 3}}+\frac{c^{2}}{\left(\gamma_{1}-\gamma_{2}\right)^{2}}+\frac{c^{2}}{\left(\gamma_{1}+\gamma_{2}\right)^{2}},
$$

где величины $\gamma_{i}$ выражаются через главные полуоси эллипсоида $A_{i}$ по формулам $\gamma_{i}=A_{i} / \sqrt{\Sigma A_{i}^{2}}$. Таким образом, система определена в первом квадранте сферы: $\left\{\gamma^{2}=1 \mid \gamma_{i}>0, i=1,2,3\right\}$, причем при $c \neq 0$ необходимо исключить диагональ $\gamma_{1}=\gamma_{2}$. Всюду внутри области определения уровень энергии $H=h$ представляет собой компактное трехмерное многообразие. 

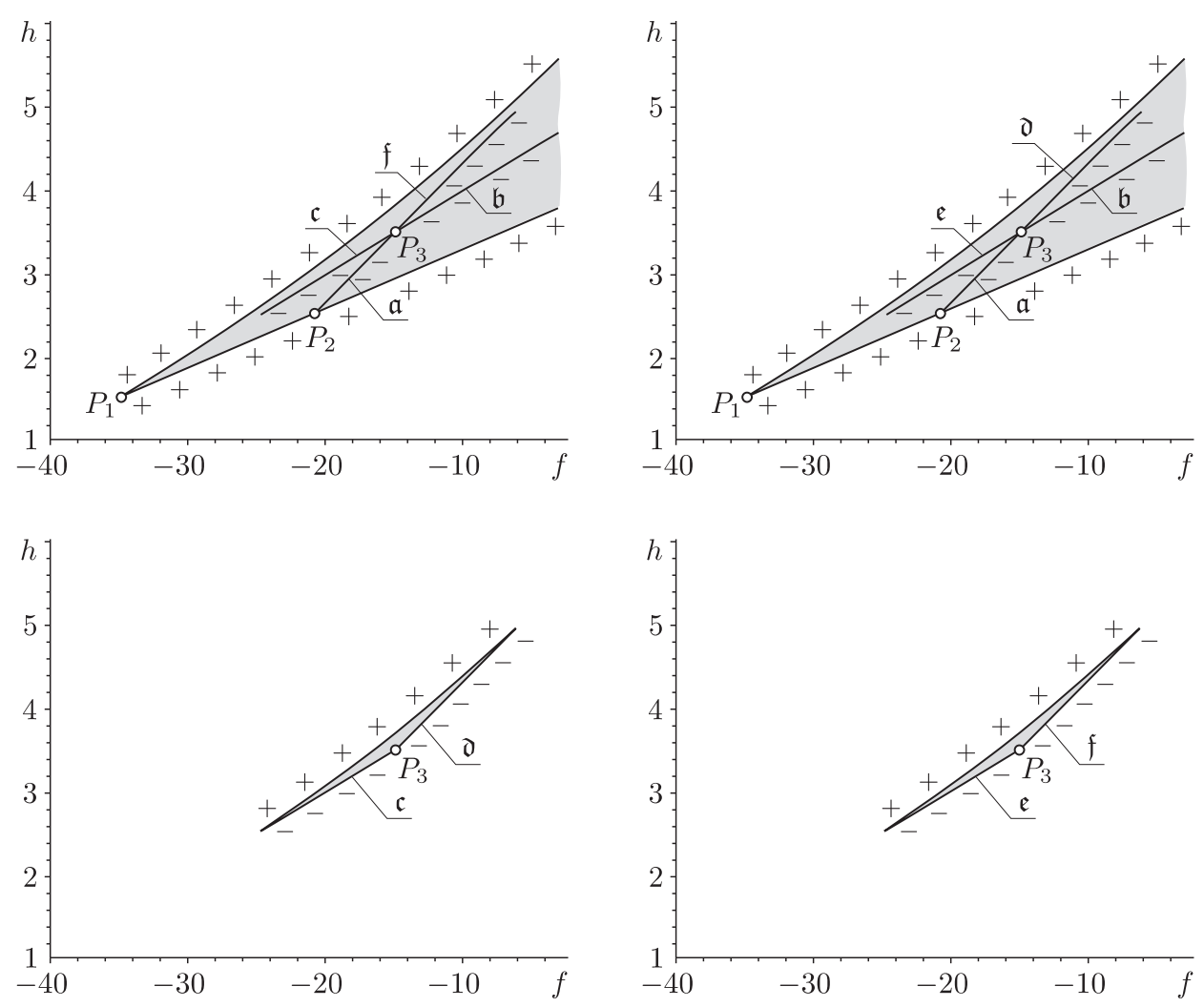

Рис. 14. Отдельные листы и порядок склейки бифуркационного комплекса при $(\mu \operatorname{det} \mathbf{I})^{-1 / 2} c<\sqrt{a_{1}-a_{3}}-\sqrt{a_{2}-a_{3}}$ : кривые, помеченные одинаковыми буквами, склеиваются между собой.

Система (24) допускает интеграл шестой степени

$$
\begin{gathered}
F_{6}=\left(F_{3}+F_{c}\right)^{2}+4 \Phi\left(3 a+G \frac{\gamma_{1}^{2}}{\gamma_{3}^{2}}\right)\left(3 a+G \frac{\gamma_{2}^{2}}{\gamma_{3}^{2}}\right), \\
F_{3}=M_{1} M_{2} M_{3}-3 a\left(\gamma_{1} \gamma_{2} \gamma_{3}\right)^{1 / 3} \sum \frac{M_{i}}{\gamma_{i}}, \quad F_{c}=\frac{4 c^{2} \gamma_{1} \gamma_{2} \gamma_{3}^{2}}{\left(\gamma_{1}^{2}-\gamma_{2}^{2}\right)^{2}} M_{3}, \\
\Phi=4 c^{2}\left(\gamma_{1} \gamma_{2} \gamma_{3}\right)^{2 / 3} \frac{\gamma_{3}^{2}}{\left(\gamma_{1}^{2}-\gamma_{2}^{2}\right)^{2}}, \quad G=\left(\gamma_{1} \gamma_{2} \gamma_{3}\right)^{2 / 3} \frac{M_{1} M_{2}}{\gamma_{1} \gamma_{2}}+\Phi-3 a .
\end{gathered}
$$

При $c=0$, согласно $(25)$, имеется интеграл третьей степени $F_{3}$.

ЗАмечАниЕ. Система Гаффэ представляет собой относительно новый, интересный класс интегрируемых систем астрофизического характера, который был найден Б. Гаффэ [73], [74] и с тех пор детально исследуется аналитически в его многочисленных работах. В статье [75] предложен алгоритм редукции этой системы, первоначально связанной с динамикой расширяющегося (т. е. 


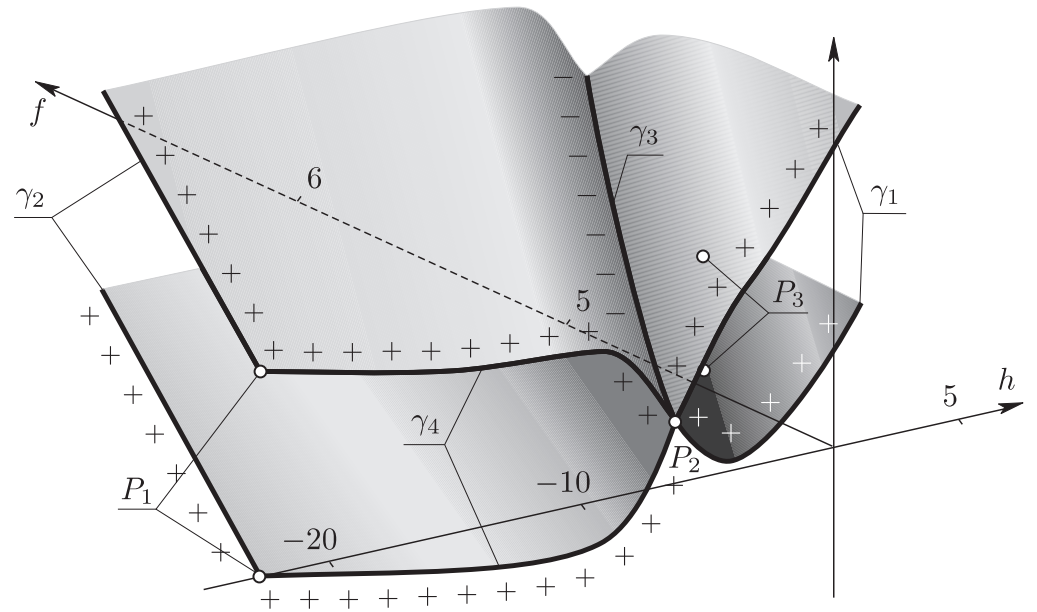

Рис. 15. Бифуркационный комплекс при $\sqrt{a_{1}-a_{3}}-\sqrt{a_{2}-a_{3}}<$ $(\mu \operatorname{det} \mathbf{I})^{-1 / 2} c<\sqrt{a_{1}-a_{3}}+\sqrt{a_{2}-a_{3}}$

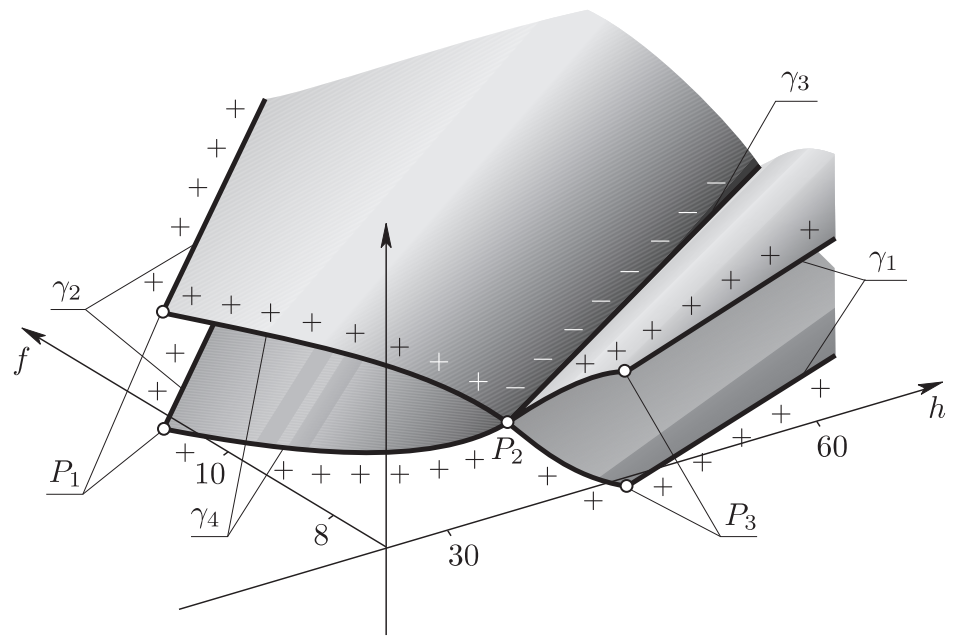

Рис. 16. Бифуркационный комплекс при $(\mu \operatorname{det} \mathbf{I})^{-1 / 2} c>\sqrt{a_{1}-a_{3}}+$ $\sqrt{a_{2}-a_{3}}$

без самогравитации) газового эллипсоида, к некоторой системе на сфере, т. е. к компактному случаю. K этой приведенной системе применимы все наши методы анализа. В данном случае они фактически являются безальтернативными, поскольку полученное Гаффэ явное решение имеет крайне сложную форму, из которой невозможно извлечь никакой информации о динамике системы. Наш подход позволяет качественно описать динамику системы и исследовать ее устойчивость.

Рассмотрим по отдельности случаи $c=0$ и $c \neq 0$; найдем критические периодические траектории и исследуем их устойчивость. 
5.1. Случай $c=0$ (интеграл третьей степени). Определим канонические переменные $\theta, p_{\theta}, \psi, p_{\psi}$ следующим образом:

$$
\begin{gathered}
\gamma_{1}=\sin \theta \sin \frac{\psi}{2}, \quad \gamma_{1}=\sin \theta \cos \frac{\psi}{2}, \quad \gamma_{3}=\cos \theta \\
M_{1}=p_{\theta} \cos \frac{\psi}{2}-2 p_{\psi} \frac{\cos \theta}{\sin \theta} \sin \frac{\psi}{2}, \quad M_{2}=-p_{\theta} \sin \frac{\psi}{2}-2 p_{\psi} \frac{\cos \theta}{\sin \theta} \cos \frac{\psi}{2}, \\
M_{3}=2 p_{\psi} .
\end{gathered}
$$

Переменные $\theta, \psi$ с точностью до множителя - это углы Эйлера; с учетом указанных выше ограничений $\theta \in(0, \pi / 2), \psi \in(0, \pi)$.

Для гамильтониана и дополнительного интеграла $F_{3}$ получим

$$
\begin{gathered}
H=\frac{1}{2} p_{\theta}^{2}+\frac{2 p_{\psi}^{2}}{\sin ^{2} \theta}+\frac{3}{2} \frac{\tilde{a}}{Q^{2}}, \quad Q=\left(\sin \psi \cos \theta \sin ^{2} \theta\right)^{1 / 3}, \quad \tilde{a}=2^{2 / 3} a \\
F_{3}=4 p_{\psi}^{2} \frac{\cos \theta}{\sin ^{2} \theta}\left(p_{\psi} \cos \theta \sin \psi-p_{\theta} \sin \theta \cos \psi\right)-p_{\theta}^{2} p_{\psi} \sin \psi \\
-\frac{3 \tilde{a}}{Q^{2}}\left(p_{\psi} \sin \psi\left(1-3 \cos ^{2} \theta\right)-p_{\theta} \cos \psi \sin \theta \cos \theta\right)
\end{gathered}
$$

Множество $S_{0}$ (неподвижные точки системы) находится из условия экстремума (в данном случае минимума) потенциала $U=\frac{1}{2} \frac{a^{2}}{Q^{2}}$. Несложно показать, что в данном случае оно состоит из одной точки:

$$
S_{0}=\left\{\psi=\frac{\pi}{2}, \theta=\arccos \frac{1}{\sqrt{3}}\right\}
$$

т. е. в исходных переменных - это точка $\gamma_{1}=\gamma_{2}=\gamma_{3}=1 / \sqrt{3}$. Подставляя эти значения $\psi$ и $\theta$ в (27), получим точку на плоскости интегралов $P_{0}=(f=0$, $\left.h=(9 / 4) 2^{1 / 3} \tilde{a}\right)$.

Множество $S_{1}$ (критические периодические траектории системы) находится из условия зависимости градиентов функций $H, F_{3}$

$$
\nabla F_{3}+\lambda \nabla H=0
$$

Семейство (или несколько семейств) искомых траекторий будем представлять в виде семейства начальных условий, из которых выходят эти траектории.

Учтем, что у всякой периодической траектории на сфере имеются точки, в которых $\dot{\theta}=0$, что эквивалентно уравнению

$$
p_{\theta}=0 .
$$

Подставляя это соотношение в систему (28), находим, что при этом выполняется также условие

$$
\psi=\frac{\pi}{2} .
$$


Разрешая оставшиеся уравнения в (28) относительно $\lambda$ и $p_{\psi}$, находим

$$
\begin{aligned}
p_{\psi}^{2} & =\frac{\tilde{a}^{2}}{4} \frac{\left(1-3 \cos ^{2} \theta_{0}\right)}{\left(\sin ^{2} \theta_{0} \cos \theta_{0}\right)^{2 / 3}} \frac{1}{\eta}, \quad \lambda=3 p_{\psi}\left(\eta-\cos ^{2} \theta_{0}\right), \\
\eta_{ \pm} & =-\frac{1}{3} \frac{2-\left(1-3 \cos ^{2} \theta_{0}\right)^{2} \pm \sqrt{4-3\left(1-3 \cos ^{2} \theta-0\right)^{2}}}{1-3 \cos ^{2} \theta_{0}},
\end{aligned}
$$

где $\theta_{0}$, параметр семейства, представляет собой угол на меридиане $\psi=\pi / 2$ для начальной точки траектории.

Кроме того, необходимо еще учесть, что возможные значения $\theta_{0}$ должны удовлетворять требованию $p_{\psi}^{2}\left(\theta_{0}\right) \geqslant 0$. Вычисляя соответствующие значения первых интегралов, находим две кривые на бифуркационной диаграмме в виде

$$
\begin{gathered}
\sigma_{+}:\left(f_{+}\left(\theta_{0}\right), h_{+}\left(\theta_{0}\right)\right), \quad \theta_{0} \in\left[\theta_{*}, \theta_{1}\right], \\
\sigma_{-}:\left(f_{-}\left(\theta_{0}\right), h_{-}\left(\theta_{0}\right)\right), \quad \theta_{0} \in\left[\theta_{*}, \frac{\pi}{2}\right), \\
h_{ \pm}=\frac{\tilde{a}^{2}}{2} \frac{1 \pm \sqrt{4-3\left(1-3 \cos ^{2} \theta_{0}\right)^{2}}}{\left(\sin ^{2} \theta_{0} \cos \theta_{0}\right)^{2 / 3} \cos ^{2} \theta_{0}\left(3 \cos ^{2} \theta_{0}-2\right)}, \\
f_{ \pm}=\frac{p_{+}}{\eta} \frac{\left(1-3 \cos ^{2} \theta_{0}\right)\left(1-2 \cos ^{2} \theta_{0}\right) \pm \sqrt{4-3\left(1-\cos ^{2} \theta_{0}\right)}}{\left(\sin ^{2} \theta_{0} \cos \theta_{0}\right)^{2 / 3}}
\end{gathered}
$$

так что бифуркационное множество $\sum_{1}$ есть $\sigma_{+} \cup \sigma_{-}$, где $\theta_{*}=\arccos (1 / \sqrt{3})$, $\theta_{1}=\arccos (\sqrt{3+2 \sqrt{3}} / 2)$ (в этой точке обращается в нуль подкоренное выражение в формуле (29)). Соответствующая бифуркационная диаграмма приведена на рис. 17, а характерный вид соответствующих периодических решений - на рис. 18.

С физической точки зрения точке $P_{0}$ соответствует сферически симметричное расширение газового облака, а решениям на ветвях диаграммы - такое расширение области, при котором его относительные размеры меняются периодическим образом.

Бифуркационный комплекс в этом случае состоит из единственного листа; таким образом, все критические периодические решения являются устойчивыми, других устойчивых решений не существует.

5.2. Случай $c \neq 0$ (интеграл шестой степени). Снова воспользуемся каноническими переменными (26), при этом потенциал системы (24) имеет особенность (обращается в бесконечность) на меридиане $\varphi=\pi / 2$ (т. е. $\gamma_{1}=\gamma_{2}$ ). Вследствие того, что система инвариантна по отношению к зеркальным отражениям относительно меридиана $\varphi=\pi / 2$, в этом случае достаточно рассмотреть область $\theta \in(0, \pi / 2), \varphi \in(0, \pi / 2)$.

Сделаем замену переменных, приводящую дальнейшие уравнения к алгебраической форме

$$
R=\cos ^{2} \theta, \quad S=\left(\sin \psi \cos \theta \sin ^{2} \theta\right)^{1 / 3},
$$

при этом область возможных движений системы ограничена отрезком прямой $S=0, R \in(0,1)$ и кривой $R(1-R)^{2}-S^{3}=0$, пересекающей прямую $S=0$ в точках $R=0$ и $R=1$ (см. рис. 19). 


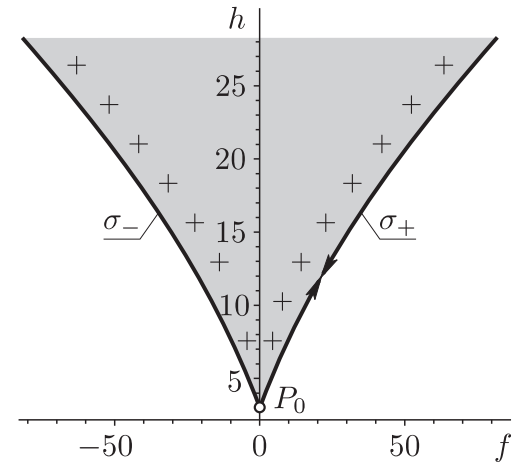

Рис. 17. Бифуркационная диаграмма системы Гаффэ при $c=0$ (интеграл третьей степени) и $\tilde{a}=1$

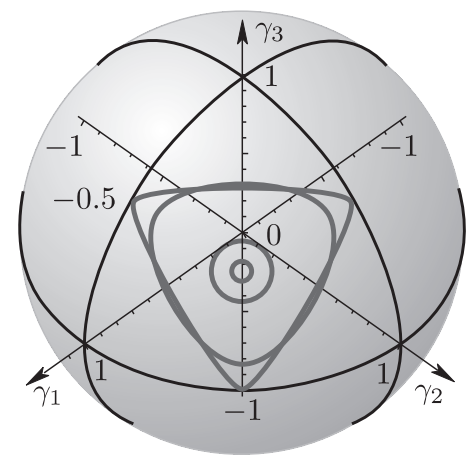

Рис. 18. Характерный вид критических периодических решений системы Гаффэ при $c=0$

$\mathbf{M н о ж е с т в о ~} \mathbf{S}_{0}$ (неподвижные точки) находится из условия минимума потенциала системы (24)

$$
U=\frac{3}{2} \frac{\tilde{a}}{S}+2 c^{2} \frac{R(1-R)}{R(1-R)^{2}-S^{3}},
$$

которые в переменных (30) могут быть приведены к системе двух алгебраических уравнений следующего вида:

$$
S^{3}-\frac{R^{2}(1-R)^{2}}{1-2 R}=0, \quad 4 S c^{2}-\frac{(1-3 R)^{2}(1-R)}{1-2 R} \tilde{a}=0 .
$$

Несложно показать, что при всех значениях $c^{2}>0$, и $\tilde{a}>0$ в области возможных движений имеется лишь одно решение этой системы: $P_{0}=\left(R_{0}, S_{0}\right)$.

Следовательно, внутри области $\theta \in(0, \pi / 2), \psi \in(0, \pi / 4)$ находится единственная точка экстремума (в данном случае минимума) потенциала (31).

Множество $\mathbf{S}_{1}$ (критические периодические решения), как и выше, находится из условия зависимости первых интегралов

$$
\nabla F_{6}+\lambda \nabla H=0
$$

Как показывают численные эксперименты, справедлива следующая гипотеза.

ГиПотезА. При с $\neq 0$ все критические периодические решения имеют точки остановки (аналогичны маятниковым движениям).

Таким образом, для начальных условий критических траекторий в данном случае можно полагать

$$
p_{\theta}=0, \quad p_{\psi}=0 .
$$


Подставляя эти условия в уравнение (32), получим уравнение, определяющее начальные значения $R, S$ для критических траекторий, в виде

$$
\begin{aligned}
W_{c}= & -9\left(-8 R^{5}+8 R^{6}+2 S^{2} R-10 S^{3} R^{2}+20 S^{3} R^{3}-S^{6}\right) \\
& \times\left(R-2 R^{2}+R^{3}-S^{3}\right)^{3} S^{2} \tilde{a}^{3} \\
+ & 12 S^{3} R\left(16 R^{5}-32 R^{6}+16 R^{7}+10 S^{3} R^{2}-60 S^{3} R^{3}+46 S^{3} R^{4}+2 S^{6}+S^{6} R\right) \\
& \times\left(R-2 R^{2}+R^{3}-S^{3}\right)^{2} c^{2} \tilde{a}^{2}-16 S^{4} R^{3}\left(R-2 R^{2}+R^{3}-S^{3}\right) \\
& \times\left(8 R^{7}-24 R^{6}+24 R^{5}-8 R^{4}+32 S^{3} R^{4}-62 S^{3} R^{3}+30 S^{3} R^{2}\right. \\
+ & \left.5 S^{6} R-4 S^{6}\right) \tilde{a} c^{4}+64 S^{8} R^{5}\left(-4 R^{3}+2 R^{2}+S^{3} R-2 S^{3}+2 R^{4}\right) c^{6}=0 .
\end{aligned}
$$

Функция $W_{c}$ имеет внутри области возможных движений единственную седловую точку, в которой она также обращается в нуль (см. рис. 19), эта точка совпадает с точкой минимума потенциала (31). Из нее выходят четыре кривые, которые соответствуют двум парам точек остановки двух семейств критических периодических траекторий (см. рис. 19).

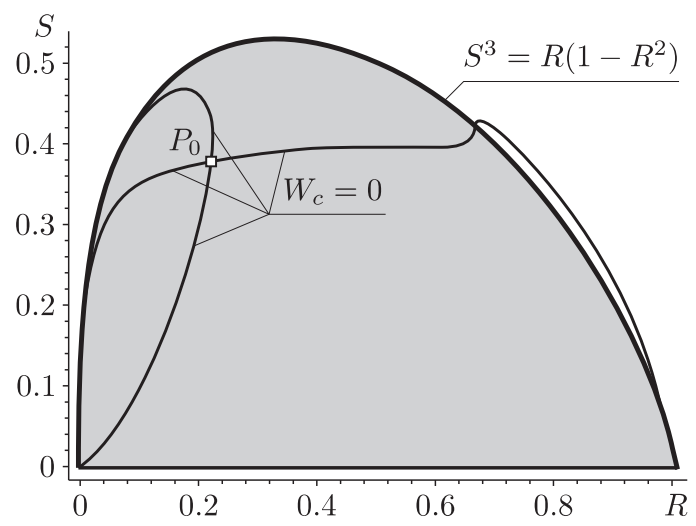

Рис. 19. Характерный вид множества $W_{c}(R, S)=0(\tilde{a}=1, c=0.7)$

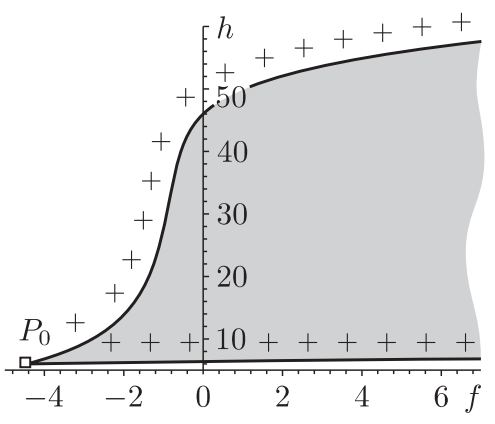

a)

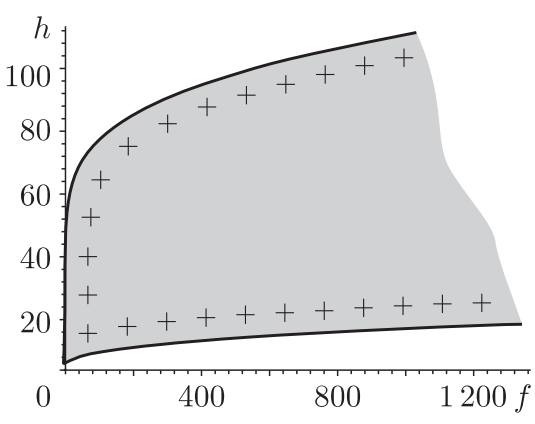

b)

Рис. 20. Характерный вид бифуркационной диаграммы системы Гаффэ при $\tilde{a}=1, c=0.7$ (на рис. b) изображен увеличенный фрагмент участка на рис. а)) 
Вычисляя значения первых интегралов для точек множества $W_{c}=0$, получим бифуркационную диаграмму системы, изображенную на рис. 20. Как и в предыдущем случае, бифуркационный комплекс в данном случае состоит из единственного листа, следовательно, все критические периодические решения в данном случае устойчивы, других устойчивых периодических траекторий не существует.

\section{6. Заключение}

В заключение отметим некоторые перспективы дальнейшего развития этого подхода как в общетеоретическом, так и в прикладном направлении.

1. С общетеоретической точки зрения, было бы интересно попытаться распространить эту теорию на системы с тремя и более степенями свободы [76]-[78], а также на системы с некомпактными интегральными многообразиями (такие системы особенно часто встречаются в небесной механике, см., например, [25], [79]). Очевидно, что какое-то прямое обобщение здесь получить очень сложно.

2. Практический аспект связан с расширением области применения данных методов. Они могут быть применены для качественного анализа многих конкретных задач из различных областей механики и математической физики, например, в задачах неголономной механики, где, оказывается, существует целая иерархия динамического поведения интегрируемых систем [53]. Динамическое поведение может быть близко к гамильтонову, а может отличаться. Так, в неголономной механике может отсутствовать инвариантная мера, хотя такое же слоение на интегральные двумерные компактные многообразия может существовать. Недавно наш метод был апробирован для нахождения частных решений задачи о шаре Чаплыгина с гиростатом [49] и определения их устойчивости. Было бы интересно исследовать таким образом и другие задачи из неголономной механики, например, шаровой подвес Федорова, движение резинового тела и др. Данные топологические методы также приложимы к анализу задач вихревой динамики, в частности, задач о движении точечных вихрей на плоскости и сфере [80], а также динамики твердого тела, взаимодействующего с точечными вихрями [81], [82].

3. Кроме того, было бы интересно исследовать связь между нашими методами анализа устойчивости и методами Смейла, которые стоило бы изложить в более приемлемой для механиков форме. Методы Смейла [1] большей частью применимы к неинтегрируемым ситуациям. Возможно, эти два подхода в некотором смысле могут сочетаться на системах, близких к интегрируемым.

Авторы выражают признательность за последние замечания и обсуждения результатов работы Б. С. Бардину, С. М. Гусейн-Заде, В. В. Козлову, А. П. Маркееву, А. И. Нейштадту, С. М. Рамоданову, М. П. Харламову.

\section{Приложение А}

A.1. Доказательство теоремы 7. Итак, мы хотим доказать следующие свойства изолированных семейств замкнутых критических траекторий, отображающихся на гладкие ветви бифуркационной диаграммы: 
1) если в таком семействе содержится хотя бы одна невырожденная траектория, то таковыми будут почти все траектории семейства;

2) если хотя бъ одна траектория в семействе имеет эллиптический тип, то все траектории семейства устойчивы, напротив, если хотя бы одна траектория в семействе имеет гиперболический тип, то все траектории семейства неустойчивы.

Тот факт, что почти все траектории в семействе невырождены, если невырождена хотя бы одна, является очевидным следствием аналитичности. Более того, вырожденные траектории, если они имеются, обязаны быть изолированными внутри данного семейства.

Вторая часть утверждения является простой переформулировкой некоторых фактов из теории особенностей гладких отображений. Его доказательство основано в основном на топологических соображениях.

Во-первых, для удобства мы заменим интеграл $F$ на некоторую подходящую комбинацию $F$ и $H$ так, чтобы бифуркационная кривая стала горизонтальной прямой $F=0$. Очевидно, что так можно сделать. Далее будем работать в окрестности $\gamma$, одной из периодических траекторий семейства.

Чтобы описать аналитически характер взаимного поведения функций $F$ и $H$ в окрестности некоторой точки $P_{0} \in \gamma$, мы заметим, что можно выбрать канонические координаты $p_{1}, q_{1}, p_{2}, q_{2}$ таким образом, чтобы $H=p_{1}$. Тогда, очевидно, $F=F\left(p_{1}, p_{2}, q_{2}\right)$, т. е. $F$ не зависит от $q_{2}$ (поскольку коммутирует с $H$ ). Параметром семейства является функция $H=p_{1}$, именно так мы и будем рассматривать эту переменную, записывая функцию $F\left(p_{1}, p_{2}, q_{2}\right)$ в виде $F_{p_{1}}\left(p_{2}, q_{2}\right)$. Предположения теоремы говорят нам, что при каждом значении параметра $p_{1}$ функция $F=F_{p_{1}}\left(p_{2}, q_{2}\right)$ имеет ровно одну критическую точку, причем значение функции в этой точке равно нулю (это следует из вида бифуркационной кривой).

Таким образом, ситуация, которую мы изучаем, очень хорошо моделируется в обычном трехмерном пространстве. Мы изучаем стандартный вопрос из теории особенностей: поведение особых точек при изменении параметра.

Условие невырожденности означает, что $F=F_{p_{1}}\left(p_{2}, q_{2}\right)$ является функцией Морса, т. е. имеет невырожденный гессиан в особой точке:

$$
D=\operatorname{det}\left(\begin{array}{cc}
\frac{\partial^{2} F}{\partial p_{2}^{2}} & \frac{\partial^{2} F}{\partial p_{2} \partial q_{2}} \\
\frac{\partial^{2} F}{\partial p_{2} \partial q_{2}} & \frac{\partial^{2} F}{\partial q_{2}^{2}}
\end{array}\right) \neq 0 .
$$

Эллиптический тип соответствует случаю $D>0$, гиперболический - случаю $D<0$.

Прежде всего объясним, почему в описываемой ситуации гиперболические невырожденные точки не могут стать эллиптическими (это стандартный факт из теории особенностей). Другими словами, $D$ не может изменить знак.

На каждом уровне $p_{1}=$ const имеется ровно одна изолированная особая точ$\kappa a$. Рассматривая $p_{1}$ как параметр, возьмем окружность малого радиуса $\delta$ с центром в такой точке. При каждом значении $p_{1}$ рассмотрим векторное поле 
$\operatorname{grad} F_{p_{1}}\left(p_{2}, q_{2}\right)$ на плоскости $p_{1}=$ const и вычислим его индекс относительно рассматриваемой особой точки (т. е. число оборотов при обходе вдоль соответствующей окружности малого радиуса). Хорошо известно, что при непрерывном изменении параметра этот индекс меняться не может. А у эллиптической и гиперболической точек он разный (у эллиптической ind $=+1$, у гиперболической ind $=-1$ ). Таким образом, смена типа внутри рассматриваемого семейства невозможна.

ЗАмечАниЕ. Следует иметь в виду, что существуют, разумеется, такие непрерывные семейства траекторий, в которых тип меняется с гиперболического на Эллиптический. Эта ситуация является совершенно типичной и в теории особенностей, и в интегрируемых системах. Она описывается хорошо известной формулой:

$$
F_{p_{1}}\left(p_{2}, q_{2}\right)=p_{2}^{2}+p_{1} q_{2}-q_{2}^{2} .
$$

Здесь мы тоже имеем однопараметрическое семейство особых точек, задаваемое уравнениями $p_{2}=0, p_{1}=3 q_{2}^{2}$. Причем при $q_{2}>0$ мы получаем эллиптическую особенность, а при $q_{2}<0$ - гиперболическую. В точке $q_{2}=0$ происходит смена типа.

Никакого противоречия с доказанным утверждением нет. Отличие от нашего случая здесь в том, что $p_{1}$ не может рассматриваться как параметр семейства особых точек. Если же рассматривать $p_{1}$ как параметр семейства функций, то произошедшее событие следует интерпретировать так: эллиптическая и гиперболическая точки сближаются, затем сливаются в одну вырожденную особую точку (иногда ее называют параболической), после чего особенность исчезает. При интегральном отображении такое семейство переходит в касп (точка возврата), а не в гладкую кривую бифуркационной диаграммы. В частности, гамильтониан не может служить в качестве хорошего параметра такого семейства.

Итак, мы хотим доказать следующий факт. Пусть имеется семейство периодических критических траекторий, про которые известно, что почти все они являются невырожденными (т. е. либо гиперболическими, либо эллиптическими), однако могут встречаться отдельные вырожденные траектории. Мы хотим показать, что при вырождении ни потери, ни появления устойчивости в рассматриваемой нами ситуации произойти не может: вырожденные траектории, расположенные в эллиптическом семействе, будут устойчивыми, а в гиперболическом - неустойчивыми.

Итак, рассмотрим одну из таких вырожденных траекторий. Во введенных нами выше обозначениях этой траектории соответствует изолированная особая точка функции $F_{p_{1}}\left(p_{2}, q_{2}\right)$. Переменная $p_{1}$ рассматривается как параметр, причем в данной точке (т.е. при некотором конкретном значении параметра $p_{1}$ ) особенность вырождена, а при всех остальных близких значениях параметра является морсовской.

Как и выше, мы рассмотрим векторное поле $\operatorname{grad} F_{p_{1}}$ и его индекс в данной точке. Как мы только что объяснили, в гиперболическом семействе ind $=-1$, а в эллиптическом ind $=+1$, причем эти равенства остаются справедливыми и для вырожденных траекторий (по соображениям непрерывности). 
После этого замечания доказываемый нами факт становится очевидным следствием следующего общего и несложного утверждения из теории особенностей.

ПредЛОЖенИЕ 3. Пусть $f(x, y)$ - вещественно аналитическая функиия двух переменных, $(0,0)$ - ее изолированная особая точка и $f(0,0)=0$.

Если индекс векторного поля grad $f$ в этой точке равен +1 , то локально точка $(0,0)$ является единственным решением уравнения $f(x, y)=0$ и функция $f$ имеет в данной точке локальный минимум или локальный максимум.

Напротив, если индекс векторного поля grad $f$ в этой точке равен -1, то множество $\{f(x, y)=0\}$ содержит помимо $(0,0)$ и другие точки. Более того, в этом случае это множество одномерно и представляет собой набор кривых, “втыкающихся" в точку $(0,0)$.

Доказываемое нами утверждение теперь является очевидной комбинацией этого предложения и теоремы 6. Действительно, согласно теореме 6 , нам достаточно посмотреть на связную компоненту интегрального многообразия, содержащего данную траекторию. Если эта связная компонента совпадает с самой траекторией, то мы имеем устойчивость, если содержит что-то еще, то неустойчивость. Во введенных нами канонических переменных интегральное подмногообразие локально задается двумя простыми уравнениями

$$
H=p_{1}=\text { const } \quad \text { и } \quad F=F_{p_{1}}\left(p_{2}, q_{2}\right)=0 .
$$

Оно совпадает с траекторией тогда и только тогда, когда при соответствующем фиксированном значении параметра $p_{1}$ решение уравнения $F=F_{p_{1}}\left(p_{2}, q_{2}\right)=0$ состоит из единственной точки (эту точку естественно представлять себе как пересечение траектории с сечением Паункаре).

Но предложение 3 попросту говорит, что выполнение этого последнего условия полностью контролируется индексом, который при вырождении измениться не может; невырожденность при этом никакой существенной роли не играет. Тем самым в рассматриваемых нами семействах все траектории либо одновременно являются устойчивыми, либо одновременно являются неустойчивыми. Теорема 7 доказана.

A.2. Методы проверки невырожденности. В заключение мы обсудим несколько различных способов проверки невырожденности и определения типа замкнутых траекторий.

Как известно, проверка условия невырожденности и определение типа периодических траекторий через мультипликаторы - довольно затруднительная задача: требуется сначала выписать уравнения в вариациях, затем их решить и уже затем рассмотреть собственные значения фундаментального решения.

Определение невырожденности в смысле теории особенностей представляется более удобным с технической точки зрения, поскольку требует проверки некоторого условия на собственные значения некоторой матрицы лишь в одной точке, при этом нет необходимости интегрировать систему (линеаризованную или исходную). Согласно этому определению, невырожденность траектории в этом смысле является локальным условием: для его проверки нам не нуж- 
но знать поведение системы и интегралов вдоль всей траектории, достаточно посмотреть, что происходит лишь в одной из ее точек.

ЗАмечАниЕ. Не следует, разумеется, забывать, что невырожденность в смысле теории особенностей имеет смысл лишь для интегрируемых систем.

Опишем подробнее два метода.

1. Можно просто следовать определению, вычисляя явно ограничение интеграла $F$ на изоэнергетическое двумерное сечение Пуанкаре $\mathscr{N}_{h}$. На практике удобно поступать следующим образом.

Пусть $x_{1}, x_{2}, x_{3}, x_{4}$ - некоторые (не обязательно канонические) координаты на симплектическом многообразии $\mathscr{M}^{4}$ в окрестности рассматриваемой точки $\mathbf{x}_{0} \in \gamma$. Тогда в качестве локальных координат на изоэнергетическом уровне $H=h$ можно взять три из них, скажем, $x_{1}, x_{2}, x_{3}$ для определенности. Четвертая координата будет тогда некоторой гладкой функцией $x_{4}=x_{4}\left(x_{1}, x_{2}, x_{3}\right)$. После подстановки

$$
F\left(x_{1}, x_{2}, x_{3}, x_{4}\left(x_{1}, x_{2}, x_{3}\right)\right)=\widetilde{F}\left(x_{1}, x_{2}, x_{3}\right)=\left.F\right|_{\{H=h\}}
$$

мы получаем в точности ограничение интеграла $F$ на рассматриваемый изоэнергетический уровень. Теперь можно поступить двумя способами.

Первый способ состоит в нахождении $(3 \times 3)$-матрицы

$$
\boldsymbol{\Gamma}_{3}\left(\mathbf{x}_{\mathbf{0}}\right)=\mathrm{d}^{2} \widetilde{F}=\left\|\frac{\partial \widetilde{F}}{\partial x_{i} \partial x_{j}}\right\|, \quad 1 \leqslant i, j \leqslant 3 .
$$

Периодическая траектория $\gamma$ невырождена тогда и только тогда, когда $\operatorname{rank} \boldsymbol{\Gamma}_{3}\left(\mathbf{x}_{0}\right)=2$. Если ненулевые собственные значения $\boldsymbol{\Gamma}_{3}\left(\mathbf{x}_{0}\right)$ одного знака, то $\gamma$ - эллиптическая, в противном случае $\gamma$ - гиперболическая.

Ясно, что для определения знаков собственных значений достаточно рассмотреть лишь один отличный от нуля диагональный $(2 \times 2)$-минор: если он положительный, то $\gamma$ - эллиптическая, если отрицательный, то $\gamma$ - гиперболическая.

Второй способ заключается в том, чтобы в точности следовать исходному определению, выбирая двумерное изоэнергетическое сечение Пуанкаре $\mathscr{N}_{h}$ и ограничивая на него интеграл $F$. Для этого, как правило, достаточно зафиксировать одну из трех оставшихся координат, например, $x_{3}$ для определенности, так что

$$
\left.F\right|_{\mathscr{N}_{h}}=\left.\widetilde{F}\left(x_{1}, x_{2}, x_{3}\right)\right|_{x_{3}=\mathrm{const}}=\widetilde{\widetilde{F}}\left(x_{1}, x_{2}\right) .
$$

Заметим, что координата сечения $x_{3}$ должна быть выбрана так, чтобы соответствующая компонента исходного гамильтонова векторного поля $X_{H}$ была отлична от нуля, это условие гарантирует трансверсальность сечения. Далее вычисляем $(2 \times 2)$-матрицу

$$
\boldsymbol{\Gamma}_{2}\left(\mathbf{x}_{\mathbf{0}}\right)=\mathrm{d}^{2} \widetilde{\widetilde{F}}=\left\|\frac{\partial \widetilde{\widetilde{F}}}{\partial x_{i} \partial x_{j}}\right\|, \quad 1 \leqslant i, j \leqslant 2 .
$$


Если $\operatorname{det} \boldsymbol{\Gamma}_{2} \neq 0$, то рассматриваемая траектория невырождена; при этом если $\operatorname{det} \boldsymbol{\Gamma}_{2}>0$, то траектория - эллиптическая,

если $\operatorname{det} \boldsymbol{\Gamma}_{2}<0$, то траектория - гиперболическая.

2. Другой метод проверки удобнее использовать, когда гамильтониан и интеграл заданы на объемлющем пуассоновом многообразии (как правило, как функции не четырех, а большего числа переменных). При этом необходимо использовать имеющуюся пуассонову структуру (заметим, что выше мы ее никак не использовали), что позволит, в частности, избежать явного построения сечения Пуанкаре.

В этом случае для произвольной точки $\mathbf{x}_{0} \in \gamma$ вычислим градиенты интегралов $H, F$, которые вследствие критичности траектории $\gamma$ удовлетворяют соотношению

$$
\frac{\partial H}{\partial \mathbf{x}}=\lambda\left(\mathbf{x}_{0}\right) \frac{\partial F}{\partial \mathbf{x}}+\sum_{\alpha} \mu_{\alpha}\left(\mathbf{x}_{0}\right) \frac{\partial K_{\alpha}}{\partial \mathbf{x}}
$$

где $K_{i}-$ функция Казимира.

С помощью этого соотношения находим соответствующий неопределенный множитель $\lambda\left(\mathbf{x}_{0}\right)$ и затем вычисляем квадратичную форму

$$
\boldsymbol{\Gamma}\left(\mathbf{x}_{0}\right)=\left\|\frac{\partial^{2} H}{\partial x_{i} \partial x_{j}}\right\|-\lambda\left(\mathbf{x}_{0}\right)\left\|\frac{\partial^{2} F}{\partial x_{i} \partial x_{j}}\right\|-\sum_{\alpha} \mu_{\alpha}\left(\mathbf{x}_{0}\right) \frac{\partial^{2} K_{\alpha}}{\partial x_{i} \partial x_{j}} .
$$

При этом гамильтоновы векторные поля $X_{H}$ и $X_{F}$, порожденные гамильтонианом и интегралом, являются линейно зависимыми на $\gamma$, так что $X_{H}\left(\mathbf{x}_{0}\right)+$ $\lambda X_{F}\left(\mathbf{x}_{0}\right)=0$. Линеаризация векторного поля $X_{H}+\lambda X_{F}$ в точке $\mathbf{x}_{0}$ задается линейным оператором вида

$$
\mathbf{J} \boldsymbol{\Gamma}\left(\mathbf{x}_{0}\right)
$$

где $\mathbf{J}$ - пуассонова структура, но теперь $x_{i}$ являются координатами на пуассоновом многообразии.

Периодическая траектория $\gamma$ невырождена тогда и только тогда, когда $\operatorname{rank} \mathbf{J} \boldsymbol{\Gamma}=2$. Если отличные от нуля собственные значения матрицы $\mathbf{J}$ вещественные, то $\gamma$ - гиперболическая, если чисто мнимые, то $\gamma$ - эллиптическая.

ЗАмЕчАниЕ. Оператор линеаризации $\mathbf{J \Gamma ~ о п р е д е л е н ~ н а ~ к а с а т е л ь н о м ~ п р о - ~}$ странстве ко всему пуассонову многообразию. В частности, он задается матрицей размера $4+k$, где $k$ - коразмерность симплектических листов (т. е. число казимиров). Однако при этом подходе нет никакой нужды в рассмотрении двумерного изоэнергетического сечения Пуанкаре, так как при умножении на матрицу пуассоновой структуры все собственные значения, за исключением, быть может, двух, обращаются в нуль.

Полезно также отметить, что характеристический многочлен оператора линеаризации имеет вид $\operatorname{det}(\mathbf{J} \boldsymbol{\Gamma}-t \mathbf{E})= \pm t^{2+k}\left(t^{2}+a\right)$, где единственный нетривиальный коэффициент $a$ является суммой диагональных миноров размера 2 . Если этот коэффициент положительный, то мы имеем эллиптическую траекторию, если отрицательный - гиперболическую (наконец, если $а$ равен нулю, то рассматриваемая критическая траектория является вырожденной). 
Таким образом, если нам требуется проверить невырожденность для конкретной траектории, то задача сводится к проверке знака некоторого числа, которое вычисляется при помощи совершенно явной формулы. Предложенный алгоритм вполне подходит и для компьютерных вычислений.

\section{Список литературы}

[1] С. Смейл, "Топология и механика", УМН, 27:2 (1972), 77-123; пер. с англ.: S. Smale, "Topology and mechanics. I", Invent. Math., 10:4 (1970), 305-331; S. Smale, "Topology and mechanics. II. The planar n-body problem", Invent. Math., 11:1 (1970), 45-64.

[2] В.В. Козлов, “Топологические препятствия к интегрируемости натуральных механических систем", Докл. АН СССР, 249:6 (1979), 1299-1302; англ. пер.: V.V. Kozlov, "Topological obstacles to the integrability of natural mechanical systems", Soviet Math. Dokl., 20:6 (1970), 1413-1415.

[3] В. В. Козлов, "Интегрируемость и неинтегрируемость в гамильтоновой механике", УМН, 38:1 (1983), 3-67; англ. пер.: V. V. Kozlov, "Integrability and non-integrability in Hamiltonian mechanics", Russian Math. Surveys, 38:1 (1983), 1-76.

[4] В. В. Козлов, Симметрии, топология и резонансы в гамильтоновой механике, Изд-во УГУ, Ижевск, 1995, ISBN: 5-7029-0126-6, 429 с.; англ. пер.: V. V. Kozlov, Symmetries, topology and resonances in Hamiltonian mechanics, Ergeb. Math. Grenzgeb. (3), 31, Springer-Verlag, Berlin, 1996, ISBN: 3-540-57039-X, 378 pp.

[5] А. Т. Фоменко, Симплектическая геометрия. Методы и приложения, Изд-во МГУ, М., 1988, ISBN: 5-211-00083-8, 414 с.; англ. пер.: А. Т. Fomenko, Symplectic geometry, Adv. Stud. Contemp. Math., 5, Gordon and Breach, New York, 1988, ISBN: 2-88124-657-5, 387 pp.

[6] А. Т. Фоменко, “Топология поверхностей постоянной энергии интегрируемых гамильтоновых систем и препятствия к интегрируемости”, Изв. АН СССР. Сер. матем., 50:6 (1986), 1276-1307; англ. пер.: А. Т. Fomenko, "The topology of surfaces of constant energy in integrable Hamiltonian systems, and obstructions to integrability", Math. USSR-Izv., 29:3 (1987), 629-658.

[7] А. Т. Фоменко, Х. Цишанг, “Топологический инвариант и критерий эквивалентности интегрируемых гамильтоновых систем с двумя степенями свободы”, Изв. АН СССР. Сер. матем., 54:3 (1990), 546-575; англ. пер.: А. T. Fomenko, Kh. Tsishang, "Topological invariant and a criterion for the equivalence of integrable Hamiltonian systems with two degrees of freedom", Math. USSR-Izv., 36:3 (1991), 567-596.

[8] Т. И. Погосян, М.П. Харламов, "Бифуркационное множество и интегральные многообразия задачи о движении твердого тела в линейном поле сил", ПММ, 43:3 (1979), 419-428; англ. пер.: T. I. Pogosian, M. P. Kharlamov, "Bifurcation set and integral manifolds of the problem concerning the motion of a rigid body in a linear force field", J. Appl. Math. Mech., 43:3 (1979), 452-462.

[9] Т. И. Погосян, "Построение бифуркационных множеств в одной задаче динамики твердого тела", Механика твердого тела, 12, Наукова думка, Киев, 1980, 9-16.

[10] Т. И. Погосян, "Области возможности движения и интегральные многообразия в задаче Клебша. Регулярный случай”, Механика твердого тела, 16, Наукова думка, Киев, 1984, 12-19.

[11] Т. И. Погосян, “Области возможности движения в задаче Клебша. Критический случай”, Механика твердого тела, 15, Наукова думка, Киев, 1983, 3-23. 
[12] Т.И. Погосян, "Области возможности движения в задаче Клебша", Механика твердого тела, 16, Наукова думка, Киев, 1984, 19-24.

[13] М. П. Харламов, Топологический анализ интегрируемых задач динамики твердого тела, Изд-во ЛГУ, Л., 1988, ISBN: 5-288-00284-3, 200 с.

[14] А.В. Болсинов, А.Т. Фоменко, Интегрируемые гамильтоновы системы. Геометрия, топология, классификация, т. 1, 2, РХД, Ижевск, 1999, ISBN: 5-70290352-8, 444 с.; англ. пер.: А. V. Bolsinov, А. T. Fomenko, Integrable Hamiltonian systems. Geometry, topology, classification, Chapman \& Hall, Boca Raton, FL, 2004, ISBN: 0-415-29805-9, 730 pp.

[15] A. A. Oshemkov, "Fomenko invariants for the main integrable cases of the rigid body motion equations", Topological classification of integrable systems, Adv. Soviet Math., 6, Amer. Math. Soc., Providence, RI, 1991, 67-146.

[16] M. P. Harlamov, P. E. Ryabov, "The bifurcations of the first integrals in the case of Kowalewski-Yehia", Regul. Chaotic Dyn., 2:2 (1997), 25-40.

[17] O.E. Orel, P.E. Ryabov, "Bifurcation sets in a problem on motion of rigid body in fluid and in the generalization of this problem", Regul. Chaotic Dyn., 3:2 (1998), 82-91.

[18] П. В. Морозов, “Лиувиллева классификация интегрируемых систем случая Клебша", Матем. сб., 193:10 (2002), 113-138; англ. пер.: P. V. Morozov, "The Liouville classification of integrable systems of the Clebsch case", Sb. Math., 193:10 (2002), 1507-1533.

[19] П.В.Морозов, “Топология слоений Лиувилля случаев интегрируемости Стеклова и Соколова уравнений Кирхгофа", Матем. сб., 195:3 (2004), 69-114; англ. пер.: P. V. Morozov, "Topology of Liouville foliations in the Steklov and the Sokolov integrable cases of Kirchhoff's equations", Sb. Math., 195:3 (2004), 369-412.

[20] М. Ю. Ивочкин, “Топологический анализ движения эллипсоида по гладкой плоскости”, Матем. сб., 199:6 (2008), 85-104; англ. пер.: М. Yu. Ivochkin, "Topological analysis of the motion of an ellipsoid on a smooth plane", Sb. Math., 199:6 (2008), 871-890.

[21] Д. Б. Зотьев, "Фазовая топология первого класса Аппельрота волчка Ковалевской в магнитном поле", Фундам. и прикл. матем., 12:1 (2006), 95-128; англ. пер.: D. B. Zotev, "Phase topology of Appelrot class I of a Kowalewski top in a magnetic field", J. Math. Sci. (N. Y.), 149:1 (2008), 922-946.

[22] А. В. Болсинов, П. Х. Рихтер, А. Т. Фоменко, "Метод круговых молекул и топология волчка Ковалевской", Матем. сб., 191:2 (2000), 3-42; англ. пер.: A. V. Bolsinov, P. H. Richter, A. T. Fomenko, "The method of loop molecules and the topology of the Kovalevskaya top", Sb. Math., 191:2 (2000), 151-188.

[23] M.Radnović, V. Rom-Kedar, "Foliations of isonergy surfaces and singularities of curves", Regul. Chaotic Dyn., 13:6 (2008), 645-668.

[24] H. R. Dullin, M. Juhnke, P. H. Richter, "Action integrals and energy surfaces of the Kovalevskaya top", Internat. J. Bifur. Chaos Appl. Sci. Engrg., 4:6 (1994), 1535-1562.

[25] H. Waalkens, H. R. Dullin, P. H. Richter, "The problem of two fixed centers: bifurcations, actions, monodromy", Phys. D, 196:3-4 (2004), 265-310.

[26] E. Sinitsyn, B. Zhilinskii, "Qualitative analysis of the classical and quantum Manakov top", SIGMA Symmetry Integrability Geom. Methods Appl., 3 (2007), 046, 23 pp.; arXiv: math/arXiv:math-ph/0703045, 2007.

[27] J. J. Duistermaat, "On global action-angle coordinates", Comm. Pure Appl. Math., 33:6 (1980), 687-706. 
[28] L. H. Eliasson, "Normal forms for Hamiltonian systems with Poisson commuting integrals - elliptic case", Comment. Math. Helv., 65:1 (1990), 4-35.

[29] Л.М.Лерман, Я. Л. Уманский, "Классификация четырехмерных интегрируемых гамильтоновых систем и пуассоновских действий $\mathbb{R}^{2}$ в расширенных окрестностях простых особых точек. I", Матем. сб., 183:12 (1992), 141-176; англ. пер.: L. M. Lerman, Ya.L. Umanskii, "Classification of four-dimensional integrable Hamiltonian systems and Poisson actions of $\mathbb{R}^{2}$ in extended neighborhoods of simple singular points. I", Russian Acad. Sci. Sb. Math., 77:2 (1994), 511-542; "II", Maтем. сб., 184:4 (1993), 105-138; англ. пер.: "II", Russian Acad. Sci. Sb. Math., 78:2 (1994), 479-506; "III. Реализация", Матем. сб., 186:10 (1995), 89-102; англ. пер.: "III. Realization", Sb. Math., 186:10 (1995), 1477-1491.

[30] R. H. Cushman, L. M. Bates, Global aspects of classical integrable systems, Birkhäuser, Basel, 1997, ISBN: 3-7643-5485-2, 435 pp.

[31] T. Delzant, "Hamiltoniens périodiques et images covexes de l'application moment", Bull. Soc. Math. France, 116:3 (1988), 315-339.

[32] N.T. Zung, "Symplectic topology of integrable Hamiltonian systems. I: ArnoldLiouville with singularities", Compos. Math., 101:2 (1996), 179-215.

[33] N. T. Zung, "Symplectic topology of integrable hamiltonian systems. II: Topological classification", Compos. Math., 138:2 (2003), 125-156.

[34] И. А. Тайманов, “Топологические препятствия к интегрируемости геодезических потоков на неодносвязных многообразиях", Изв. АН СССР. Сер. матем., 51:2 (1987), 429-435; англ. пер.: I. A. Taimanov, "Topological obstructions to integrability of geodesic flows on non-simply-connected manifolds", Math. USSR-Izv., 30:2 (1988), 403-409.

[35] И. А. Тайманов, "О топологических свойствах интегрируемых геодезических потоков", Матем. заметки, 44:2 (1988), 283-284.

[36] С.В. Болотин, "Неинтегрируемость задачи $n$ центров при $n>2$ ", Вестн. Моск. ун-та. Сер. матем., мех., 1984, № 3, 65-68; англ. пер.: S. V. Bolotin, "Nonintegrability of the problem of $n$ centers for $n>2$ ", Mosc. Univ. Mech. Bull., 39:3 (1984), 65-68.

[37] С. В. Болотин, "О первых интегралах систем с гироскопическими силами", Вестн. Моск. ун-та. Сер. матем., мех., 1984, № 6, 75-82; англ. пер.: S. V. Bolotin, "First integrals of systems with gyroscopic forces", Mosc. Univ. Mech. Bull., 39:6 (1984), 20-28.

[38] A. V. Bolsinov, I. A. Taimanov, "Integrable geodesic flows with positive topological entropy", Invent. Math., 140:3 (2000), 639-650.

[39] G.P. Paternain, "On the topology of manifolds with completely integrable geodesic flows", Ergodic Theory Dynam. Systems, 12:1 (1992), 109-121.

[40] L. T. Butler, "Invariant fibrations of geodesic flows", Topology, 44:4 (2005), 769-789.

[41] L. T. Butler, "The Maslov cocycle, smooth structures and real-analytic complete integrability", Amer. J. Math., 131:5 (2009), 1311-1336; arXiv: 0708.3157v2.

[42] Б. С. Бардин, "К задаче об устойчивости маятникообразных движений твердого тела в случае Горячева-Чаплыгина", Изв. РАН. МTT, 2007, № 2, 14-21.

[43] А. З. Брюм, "Исследование орбитальной устойчивости при помощи первых интегралов", ПММ, 53:6 (1989), 873-879; англ. пер.: A.Z. Bryum, "Orbital stability analysis using first integrals", J. Appl. Math. Mech., 53:6 (1989), 689-695.

[44] А. В. Карапетян, "Инвариантные множества в задаче Горячева-Чаплыгина: существование, устойчивость и ветвление", ПММ, 70:2 (2006), 221-224; англ. пер.: 
A. V. Karapetyan, "Invariant sets in the Goryachev-Chaplygin problem: existence, stability and branching", J. Appl. Math. Mech., 70:2 (2006), 195-198.

[45] А. В. Карапетян, "Инвариантные множества в задаче Клебша-Тиссерана: существование и устойчивость", ПММ, 70:6 (2006), 959-964; англ. пер.: А. V. Karapetyan, "Invariant sets in the Clebsch-Tisserand problem: Existence and stability", J. Appl. Math. Mech., 70:6 (2006), 859-864.

[46] А. П. Маркеев, “Об устойчивости плоских движений твердого тела в случае Ковалевской", ПММ, 65:1 (2001), 51-58; англ. пер.: А. P. Markeyev, "The stability of the plane motions of a rigid body in the Kovalevskaya case", J. Appl. Math. Mech., 65:1 (2001), 47-54.

[47] А. П. Маркеев, С. В. Медведев, Т. Н. Чеховская, "К задаче об устойчивости маятниковых движений твердого тела в случае Ковалевской”, Изв. РАН. МТT, 2003, № $1,3-9$.

[48] А.П. Маркеев, "О маятникообразных движениях твердого тела в случае Горячева-Чаплыгина", ПММ, 68:2 (2004), 282-293; англ. пер.: А. P. Markeyev, "The pendulum-like motions of a rigid body in the Goryachev-Chaplygin case", J. Appl. Math. Mech., 68:2 (2004), 249-258.

[49] А. Ю. Москвин, "Шар Чаплыгина с гиростатом: особые решения", Нелинейная динамика, 5:3 (2009), 345-356; http://nd.ics.org.ru/doc/r/pdf/1547/0.

[50] В.И. Арнольд, В.В. Козлов, А.И. Нейштадт, "Математические аспекты классической и небесной механики", Итоги науки и техн. Сер. Соврем. пробл. матем. Фундам. направления, т. 3, ВИНИТИ, М., 1985, 304 с.; англ. пер.: V.I. Arnold, V. V. Kozlov, A. I. Neishtadt, "Mathematical aspects of classical and celestial mechanics. Dynamical systems, III", Encyclopaedia Math. Sci., 3, Springer, Berlin, 1993, vii-xiv+129 pp.

[51] А.В. Борисов, И.С. Мамаев, Динамика твердого тела. Гамильтоновы методы, интегрируемость, хаос, 2-е изд., НИЦ "РХД", Ижевск, 2005, ISBN: 5-93972-485-X, 576 c.

[52] Б. П. Демидович, Лекции по математической теории устойчивости, Наука, М., $1967,472 \mathrm{c.}$

[53] А.В. Борисов, И.С. Мамаев, "Законы сохранения, иерархия динамики и явное интегрирование неголономных систем", Нелинейная динамика, 4:3 (2008), 223-280; http://nd.ics.org.ru/doc/r/pdf/1312/0; англ пер.: A.V. Borisov, I. S. Mamaev, "Conservation laws, hierarchy of dynamics and explicit integration of nonholonomic systems", Regul. Chaotic Dyn., 13:5 (2008), 443-490.

[54] K. Ehlers, J. Koiller, J. Montgomery, P. Rios, "Nonholonomic systems via moving frames: Cartan equivalence and Chaplygin Hamiltonization", The breadth of symplectic and Poisson geometry, Progr. Math., 232, eds. J.E. Marsden, T. S. Ratiu, Birkhäuser, Boston, MA, 2005, 75-120.

[55] A. V. Bolsinov, "Methods of calculation of the Fomenko-Zieschang invariant", Topological classification of integrable systems, Adv. Soviet Math., 6, Amer. Math. Soc., Providence, RI, 1991, 147-183.

[56] E. Miranda, N. T. Zung, "Equivariant normal form for nondegenerate singular orbits of integrable Hamiltonian system", Ann. Sci. École Norm. Sup. (4), 37:6 (2004), 819-839.

[57] С. Смейл, “Дифференцируемые динамические системы”, УМН, 25:1 (1970), 113-185; пер. с англ.: S. Smale, "Differentiable dynamical systems", Bull. Amer. Math. Soc., 73 (1967), 747-817.

[58] H. R. Dullin, A. V. Ivanov, "Vanishing twist in the Hamiltonian Hopf bifurcation", Phys. D, 201:1-2 (2005), 27-44. 
[59] В. В. Калашников, “Типичные интегрируемые гамильтоновы системы на четырехмерном симплектическом многообразии", Изв. РАН. Сер. матем., 62:2 (1998), 49-74; англ. пер.: V. V. Kalashnikov, "Typical integrable Hamiltonian systems on a four-dimensional symplectic manifold", Izv. Math., 62:2 (1998), 261-285.

[60] С. А. Чаплыгин, "Новое частное решение задачи о вращении тяжелого твердого тела вокруг неподвижной точки", Трудъ отд. физ. наук общ-ва любителей естествознания, антропологии и этнографии, 12, вып. 1, 1904, 1-4; Собр. соч., т. 1, ГИТТЛ, М., Л., 1948, 125-132.

[61] В.В. Козлов, Методы качественного анализа в динамике твердого тела, 2-е изд., Изд-во РХД, Ижевск, 2000, ISBN: 5-93972-011-0, 248 с.

[62] А.В. Цыганов, "Разделение переменных в гиростате Ковалевской-ГорячеваЧаплыгина", ТМФ, 135:2 (2003), 240-247; англ. пер.: А. V. Tsiganov, "Separation of variables in the Kovalevskaya-Goryachev-Chaplygin gyrostat", Theoret. Math. Phys., 135:2 (2003), 651-658.

[63] A. V. Borisov, A. G. Kholmskaya, I. S. Mamaev, "S. V. Kovalevskaya top and generalizations of integrable systems", Regul. Chaotic Dyn., 6:1 (2001), 1-16.

[64] A. V. Borisov, I.S. Mamaev, "Euler-Poisson equations and integrable cases", Regul. Chaotic Dyn., 6:3 (2001), 253-276.

[65] A. Chenciner, R. Montgomery, "A remarkable periodic solution of the three-body problem in the case of equal masses", Ann. of Math. (2), 152:3 (2000), 881-901; arXiv: math/0011268, 2000.

[66] A. Chenciner, J. Gerver, R. Montgomery, C. Simo, "Simple choreographies of $N$ bodies: a preliminary study", Geometry, mechanics, and dynamics, eds. P. Newton, P. Holmes, A. Weinstein, Springer, New York, 2002, 287-308.

[67] А. В. Борисов, А. А. Килин, И. С. Мамаев, "Абсолютные и относительные хореографии в динамике твердого тела", Нелинейная динамика, 1:1 (2005), 123-141; http://nd.ics.org.ru/doc/r/pdf/734/0; англ. пер.: A.V. Borisov, A.A. Kilin, I. S. Mamaev, "Absolute and relative choreographies in rigid body dynamics", Regul. Chaotic Dyn., 13:3 (2008), 204-220.

[68] Система Клебша. Разделение переменных, явное интегрирование?, ред. А. В. Борисов, А. В. Цыганов, НИЦ "РХД", М., Ижевск, 2009, 287 с.

[69] F. Kötter, "Über die Bewegung eines festen Körpers in einer Flussigkeit. I, II", J. Reine Angew. Math., 109 (1892), 51-81, 89-111.

[70] В. Г. Марихин, В. В. Соколов, "О приведении пары квадратичных по импульсам гамильтонианов к канонической форме и о вещественном частичном разделении переменных для волчка Клебша", Нелинейная динамика, 4:3 (2008), 313-322; http://nd.ics.org.ru/doc/r/pdf/1316/0.

[71] В.А. Стеклов, "Один случай движения тяжелого твердого тела в жидкости", Труды отд. физ. наук общ-ва любителей естествознания, антропологии и этнографии, 7 (1895), 1-40.

[72] Г. Колосов, "О некоторых случаях движения твердого тела в бесконечной жидкости”, Система Клебша. Разделение переменных, явное интегрирование?, ред. А. В. Борисов, А. В. Цыганов, НИЦ "РХД”, М., Ижевск, 2009, 245-254; пер. с англ.: G. Kolossoff, "On some cases of motion of a solid in infinite liquid", Amer. J. Math., 28:4 (1906), 367-376.

[73] B. Gaffet, "Expanding gas clouds of ellipsoidal shape: new exact solutions", J. Fluid Mech., 325 (1996), 113-144.

[74] B. Gaffet, "Spinning gas clouds - without vorticity", J. Phys. A, 33:21 (2000), 3929-3946. 
[75] А. В. Борисов, А.А. Килин, И. С. Мамаев, "Многочастичные системы. Алгебра интегралов и интегрируемые случаи", Нелинейная динамика, 5:1 (2009), 53-82; http://nd.ics.org.ru/doc/r/pdf/1419/0; англ. пер.: A.V. Borisov, A. A. Kilin, I. S. Mamaev, "Multiparticle systems. The algebra of integrals and integrable cases", Regul. Chaotic Dyn., 14:1 (2009), 18-41.

[76] А.Г. Рейман, М.А. Семёнов-Тян-Шанский, “Лаксово представление со спектральным параметром для волчка Ковалевской и его обобщений”, Функ. ан. и его прил., 22:2 (1988), 87-88; англ пер.: A. G. Reiman, M. A. Semenov-Tyan-Shanskii, "Lax representation with a spectral parameter for the Kovalevskaya top and its generalizations", Funct. Anal. Appl., 22:2 (1988), 158-160.

[77] О. И. Богоявленский, "Интегрируемые уравнения Эйлера на алгебрах Ли, возникающие в задачах математической физики”, Изв. АН СССР. Сер. матем., 48:5 (1984), 883-938; англ пер.: О. I. Bogoyavlenskii, "Integrable Euler equations on Lie algebras arising in problems of mathematical physics", Math. USSR-Izv., 25:2 (1985), 207-257.

[78] О.И. Богоявленский, Опрокидывающиеся солитоны. Нелинейные интегрируемые уравнения, Наука, М., 1991, 320 с.

[79] В. М. Алексеев, “Обобщенная пространственная задача двух неподвижных центров. Классификация движений”, Бюлл. Ин-та теорет. астрономии, 10:4(117) (1965), 241-271.

[80] A. V. Bolsinov, A. V. Borisov, I.S. Mamaev, "Lie algebras in vortex dynamics and celestial mechanics - IV", Regul. Chaotic Dyn., 4:1 (1999), 23-50.

[81] A. V. Borisov, I. S. Mamaev, S. M. Ramodanov, "Motion of a circular cylinder and $n$ point vortices in a perfect fluid", Regul. Chaotic Dyn., 8:4 (2003), 449-462.

[82] A. V. Borisov, I. S. Mamaev, S. M. Ramodanov, "Dynamic interaction of point vortices and a two-dimensional cylinder", J. Math. Phys., 48:6 (2007), 065403, 9 pp.

\section{А. В. Болсинов (А. V. Bolsinov)}

им. М. В. Ломоносова;

School of Mathematics, Loughborough University, UK

E-mail: A.Bolsinov@lboro.ac.uk

\section{А. В. Борисов (А. V. Borisov)}

Институт компьютерных исследований, Ижевск

E-mail: borisov@rcd.ru

\section{И. С. Мамаев (I. S. Mamaev)}

Институт компьютерных исследований, Ижевск

E-mail: mamaev@rcd.ru 